\title{
DISCRETE TRANSPARENT BOUNDARY CONDITIONS FOR THE SCHRÖDINGER EQUATION: FAST CALCULATION, APPROXIMATION, AND STABILITY*
}

\author{
ANTON ARNOLD ${ }^{\dagger}$, MATTHIAS EHRHARDT ${ }^{\ddagger}$, AND IVAN SOFRONOV $§$
}

\begin{abstract}
We propose a way to efficiently treat the well-known transparent boundary conditions for the Schrödinger equation. Our approach is based on two ideas: to write out a discrete transparent boundary condition (DTBC) using the Crank-Nicolson finite difference scheme for the governing equation, and to approximate the discrete convolution kernel of DTBC by sum-of-exponentials for a rapid recursive calculation of the convolution.

We prove stability of the resulting initial-boundary value scheme, give error estimates for the considered approximation of the boundary condition, and illustrate the efficiency of the proposed method on several examples.
\end{abstract}

Key words. Schrödinger equation, transparent boundary conditions, discrete convolution, sum of exponentials, Padé approximations, finite difference schemes

AMS subject classifications. 2000 Subject Classification: 65M12, 35Q40, 45K05

\section{Introduction}

The development of grid algorithms for solving initial-boundary-value problems on unbounded domains involves the question of formulating boundary conditions on an (artificial) boundary that cuts off a finite computational domain from the original, infinite portion of space. These boundary conditions (BCs) are not contained in the original problem formulation: they should be obtained by a transformation of the given asymptotic conditions at infinity onto the artificial boundary. Such a transfer must provide an approximation of the solution on the unbounded domain by the solution calculated in a finite domain with an artificial boundary. If the approximation is exact, the transfer is called exact, and the corresponding artificial boundary condition is called exact or transparent. For instance, different transparent boundary conditions (TBCs) for the wave equation are derived in [47, 44, 21, 45]; examples for other evolution problems are contained in [22].

Clearly, transparent boundary conditions permit us to consider computational domains of a minimal size, and therefore, they allow potentially to construct fast algorithms of computing solutions. However, there are several problems towards this goal: the first one is connected with the derivation of the boundary conditions themselves. Secondly, the numerical treatment (approximation, stability, efficiency) of corresponding analytical formulas for the BCs is a very delicate question. As a promising approach one can consider so-called discrete transparent boundary conditions (DT$\mathrm{BCs}$ ) that are derived for a finite-difference approximation of the original evolution problem on an unbounded domain. The idea of such boundary conditions for general evolution difference equations is discussed in $[36,37]$. DTBCs keep the approximation and stability properties of the difference scheme used. However, usually there

*Received: December 6, 2002; accepted (in revised version): July 10, 2003. Communicated by Shi Jin.

${ }^{\dagger}$ Institut für Numerische Mathematik, Universität Münster, Einsteinstr. 62, D-48149 Münster, Germany (anton.arnold@math.uni-muenster.de).

${ }^{\ddagger}$ Institut für Mathematik, Technische Universität Berlin, Str. des 17. Juni 136, D-10623 Berlin, Germany (ehrhardt@math.tu-berlin.de).

$\S$ Keldysh Institute of Applied Mathematics, Russian Academy of Sciences, Miusskaya sq. 4, Moscow, Russia (sofronov@spp.keldysh.ru). 
still remains the question of relatively high computational costs required for their implementation.

This paper is concerned with the numerical treatment of DTBCs for a finitedifference scheme of the Schrödinger equation governing the time evolution of the wave function $\psi(x, y, t) \in \mathbb{C}$ in a $2 \mathrm{D}$ waveguide under the action of a given electrostatic potential $V(x, y, t) \in \mathbb{R}$.

Schrödinger equation. Consider a rectangular geometry that appears e.g. in the modeling of quantum waveguides and wave couplers (cf. [15]). In these applications the wave function $\psi(x, y, t)$ satisfies (within a good approximation) homogeneous Dirichlet BCs at the channel boundaries. Therefore, the (scaled) transient Schrödinger equation for such an (infinitely long) channel (or "lead") of a waveguide reads:

$$
\begin{aligned}
i \psi_{t} & =-\frac{1}{2} \Delta \psi+V(x, y, t) \psi, \quad x \in \mathbb{R}, 0<y<Y, t>0, \\
\psi(x, 0, t) & =\psi(x, Y, t)=0, \quad \lim _{|x| \rightarrow \infty} \psi(x, y, t)=0, \\
\psi(x, y, 0) & =\psi^{I}(x, y) .
\end{aligned}
$$

We assume that the given potential $V$ is constant outside of the computational domain $[0, X] \times[0, Y]($ cf. Figure 1.1):

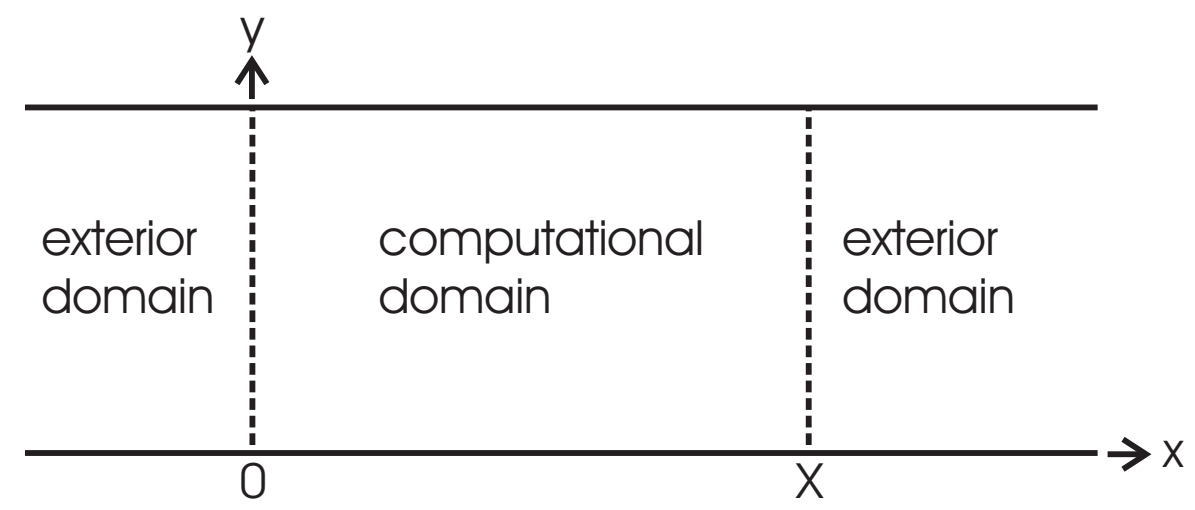

FIG. 1.1. The artificial BCs at $x=0, x=X$ cut off the exterior domains.

$$
V(x, y, t)=V_{-} \equiv \text { const } \quad \text { for } x \leq 0, \quad V(x, y, t)=V_{+} \equiv \text { const } \quad \text { for } x \geq X,
$$

$0<y<Y, t \geq 0$, and that the initial data has a compact support:

$$
\operatorname{supp} \psi^{I} \subset(0, X) \times[0, Y] \text {. }
$$

Discussions of strategies to soften these restrictions could be found in $[25,43,20]$.

Also, the computational domain is chosen here as a rectangle only for simplicity of the presentation. The geometry could be more complex, as it is the case in [15], e.g. The only constrains for our subsequent discussion of TBCs is that the exterior domains are semi-infinite strips (cf. [10] for a related stationary model). 
Equation (1.1) has also important applications in optics ("Fresnel equation", [41]) and acoustics ("parabolic equation", [48]) as a paraxial approximation to the wave equation in the frequency domain.

Analytic TBCs. Let us exemplify first analytic TBCs that can be derived for the Schrödinger equation. For simplicity, we restrict us here to the 1D case when none of the considered functions depend on $y$. These TBCs were independently derived by several authors from various application fields (cf. [34, 9, 23]; inhomogeneous extensions are analyzed in $[7,11])$. They are non-local in $t$ and read

$$
\psi_{x}(0, t)=\sqrt{\frac{2}{\pi}} e^{-\frac{\pi}{4} i} e^{-i V_{-} t} \frac{d}{d t} \int_{0}^{t} \frac{\psi(0, \tau) e^{i V_{-} \tau}}{\sqrt{t-\tau}} d \tau
$$

for the left boundary at $x=0$, and

$$
\psi_{x}(X, t)=-\sqrt{\frac{2}{\pi}} e^{-\frac{\pi}{4} i} e^{-i V_{+} t} \frac{d}{d t} \int_{0}^{t} \frac{\psi(X, \tau) e^{i V_{+} \tau}}{\sqrt{t-\tau}} d \tau
$$

for the right boundary at $x=X$.

Since TBCs are of memory-type, their numerical implementation requires to store the boundary data $\psi(0,$.$) and \psi(X,$.$) of all the past history. Moreover, the discretiza-$ tion of the left and right TBCs (1.2), (1.3) is not trivial at all and has attracted lots of attention. For the many proposed strategies of discretizations of the TBCs (1.2), (1.3) (as well as semi-discrete approaches), we refer the reader to $[3,4,9,13,31,39,41]$ and references therein. We remark also that inadequate discretizations may introduce strong numerical reflections at the boundary or render the discrete initial boundary value problem only conditionally stable, see [20] for a detailed discussion.

Difference equations. We consider a Crank-Nicolson finite difference scheme, which is one of the commonly used discretization methods for the Schrödinger equation. With the uniform grid points $x_{j}=j \Delta x, y_{k}=k \Delta y$ (where $J \Delta x=X, K \Delta y=$ $Y), t_{n}=n \Delta t$, and the approximation $\psi_{j, k, n} \sim \psi\left(x_{j}, y_{k}, t_{n}\right), j \in \mathbb{Z}, 0 \leq k \leq K, n \in$ $\mathbb{N}_{0}$, this scheme reads for the whole space problem:

$$
\begin{aligned}
-\frac{4 i}{\Delta t}\left(\psi_{j, k, n+1}-\psi_{j, k, n}\right) & \\
= & \frac{\psi_{j+1, k, n+1}-2 \psi_{j, k, n+1}+\psi_{j-1, k, n+1}}{\Delta x^{2}}+\frac{\psi_{j+1, k, n}-2 \psi_{j, k, n}+\psi_{j-1, k, n}}{\Delta x^{2}} \\
& +\frac{\psi_{j, k+1, n+1}-2 \psi_{j, k, n+1}+\psi_{j, k-1, n+1}}{\Delta y^{2}}+\frac{\psi_{j, k+1, n}-2 \psi_{j, k, n}+\psi_{j, k-1, n}}{\Delta y^{2}} \\
& -2 V_{j, k, n+\frac{1}{2}}\left(\psi_{j, k, n+1}+\psi_{j, k, n}\right), \quad j \in \mathbb{Z}, 1 \leq k \leq K-1, n \geq 0,
\end{aligned}
$$

with

$$
V_{j, k, n+\frac{1}{2}}=V\left(x_{j}, y_{k}, t_{n+\frac{1}{2}}\right) .
$$

Transparent boundary conditions are obtained by explicit solution of the equation in the two exterior domains $x \leq 0$ and $x \geq X$. In order to reduce the problem to the simpler 1D case, the Fourier method is used in $y$-direction. Due to the homogeneous Dirichlet BCs at the channel walls $(y=0, y=Y)$ we have

$$
\psi_{j, 0, n}=\psi_{j, K, n}=0, \quad j \in \mathbb{Z}, n \geq 0,
$$


and hence, use discrete Fourier transform of $\psi_{j, k, n}$ in $y$-direction with respect to sinefunctions:

$$
\psi_{j, n}^{m}:=\frac{1}{K} \sum_{k=0}^{K-1} \psi_{j, k, n} \sin \left(\frac{\pi k m}{K}\right), \quad m=1, \ldots, K-1 .
$$

The scheme (1.4), (1.5) in the two exterior domains $j \leq 0$ and $j \geq J$ then transforms into:

$$
\begin{aligned}
&-\frac{4 i}{\Delta t}\left(\psi_{j, n+1}^{m}-\psi_{j, n}^{m}\right) \\
& \quad=\frac{\psi_{j+1, n+1}^{m}-2 \psi_{j, n+1}^{m}+\psi_{j-1, n+1}^{m}}{\Delta x^{2}}+\frac{\psi_{j+1, n}^{m}-2 \psi_{j, n}^{m}+\psi_{j-1, n}^{m}}{\Delta x^{2}} \\
& \quad-2 V_{j}^{m}\left(\psi_{j, n+1}^{m}+\psi_{j, n}^{m}\right), \\
& V_{j}^{m}:=V_{j}+\frac{1-\cos \frac{\pi m}{K}}{\Delta y^{2}}, \quad j \in \mathbb{Z} \backslash[1, J-1], 1 \leq m \leq K-1, n \geq 0 .
\end{aligned}
$$

The modes $\psi^{m}, m=1, \ldots, K-1$ are independent of each other in the exterior domains since the potential $V$ is constant there. Therefore we can continue our analysis for a separate mode only.

Thus, by omitting the superscript $m$ in the notation, we will consider a discrete 1D-Schrödinger equation of the following form:

$$
\begin{aligned}
-i R\left(\psi_{j, n+1}-\psi_{j, n}\right) & =\psi_{j+1, n+1}-2 \psi_{j, n+1}+\psi_{j-1, n+1} \\
& +\psi_{j+1, n}-2 \psi_{j, n}+\psi_{j-1, n}-w V_{j, n+\frac{1}{2}}\left(\psi_{j, n+1}+\psi_{j, n}\right),
\end{aligned}
$$

where

$$
\begin{gathered}
R=\frac{4 \Delta x^{2}}{\Delta t}, \quad w=2 \Delta x^{2}, \quad V_{j, n+\frac{1}{2}}=V\left(x_{j}, t_{n+\frac{1}{2}}\right), \\
V(x, t)=V_{-} \quad \text { for } x \leq 0, \quad V(x, t)=V_{+} \quad \text { for } x \geq X, t \geq 0,
\end{gathered}
$$

with constant $V_{-}$and $V_{+}$. We remark that the spatial discretization on the computational interval $[0, X]$ can be nonuniform (e.g. adaptive in time) for our subsequent analysis.

Note two important advantages of this second order (in $\Delta x$ and $\Delta t$ ) scheme: it is unconditionally stable, and it preserves the discrete $L^{2}$-norm

$$
\left\|\psi_{n}\right\|_{2}^{2}:=\Delta x \sum_{j \in \mathbb{Z}}\left|\psi_{j, n}\right|^{2}
$$

with respect to time (these properties will be used for stability analysis in $\S 5$ ).

Discrete TBCs. Discrete transparent boundary conditions for the equation (1.8) were introduced in [5]. These DTBCs at the two boundary points $j=0$ and $j=J$ (with $X=J \Delta x$ ) can most easily be expressed for the $Z$-transformed problem:

$$
\hat{\psi}_{1}(z)=\hat{\ell}_{0}(z) \hat{\psi}_{0}(z), \quad \hat{\psi}_{J-1}(z)=\hat{\ell}_{J}(z) \hat{\psi}_{J}(z)
$$


where the $Z$-transform of the sequence $\left\{\psi_{j, n}\right\}, n \in \mathbb{N}_{0}$ (with $j$ considered fixed) is defined as the Laurent series, see [18]:

$$
\mathcal{Z}\left\{\psi_{j, n}\right\}=\hat{\psi}_{j}(z):=\sum_{n=0}^{\infty} \psi_{j, n} z^{-n}, \quad z \in \mathbb{C}
$$

The two transformed boundary kernels $\hat{\ell}_{j}(z)$ are respectively calculated as (cf. [5, 20]):

$$
\begin{gathered}
\hat{\ell}_{0}(z)=1-i \zeta_{0} \pm \sqrt{-\zeta_{0}\left(\zeta_{0}+2 i\right)}, \quad \zeta_{0}=\frac{R}{2} \frac{z-1}{z+1}+i \Delta x^{2} V_{-} \\
\hat{\ell}_{J}(z)=1-i \zeta_{J} \pm \sqrt{-\zeta_{J}\left(\zeta_{J}+2 i\right)}, \quad \zeta_{J}=\frac{R}{2} \frac{z-1}{z+1}+i \Delta x^{2} V_{+} .
\end{gathered}
$$

In order to have decaying solutions $\hat{\psi}_{j}(z)$ outside of the computational domain (i.e. for $j \rightarrow \pm \infty)$ we have to choose the branch of the square root such that $\left|\hat{\ell}_{0}(z)\right|>1$ and $\left|\hat{\ell}_{J}(z)\right|>1$. The inverse $Z$-transform of $\hat{\ell}_{j}, j=0, J$ then defines the convolution coefficients for the DTBCs:

$$
\left\{\ell_{j, n}\right\}:=\mathcal{Z}^{-1}\left\{\hat{\ell}_{j}(z)\right\}, \quad j=0, J
$$

Since the magnitude of $\ell_{j, n}$ does not decay as $n \rightarrow \infty\left(\ell_{j, n}\right.$ behaves like const $\cdot(-1)^{n}$ for large $n$ ), it is more convenient to use a modified formulation of the DTBCs (cf. [20]). We introduce

$$
\hat{s}_{j}(z):=\frac{z+1}{z} \hat{\ell}_{j}(z), \quad j=0, J
$$

and

$$
\left\{s_{j, n}\right\}=\mathcal{Z}^{-1}\left\{\hat{s}_{j}(z)\right\}
$$

which satisfy

$$
s_{j, 0}=\ell_{j, 0}, \quad s_{j, n}=\ell_{j, n}+\ell_{j, n-1}=\mathcal{O}\left(n^{-\frac{3}{2}}\right), \quad n \geq 1 .
$$

The corresponding Laurent series of

$$
\hat{s}_{j}(z)=\sum_{n=0}^{\infty} s_{j, n} z^{-n}
$$

converges (and is continuous) for $|z| \geq 1$ because of the decay (1.15).

In physical space the DTBCs then read (cf. Th. 3.8 in [20]):

$$
\begin{aligned}
\psi_{1, n}-s_{0,0} \psi_{0, n} & =\sum_{k=1}^{n-1} s_{0, n-k} \psi_{0, k}-\psi_{1, n-1}, \quad n \geq 1, \\
\psi_{J-1, n}-s_{J, 0} \psi_{J, n} & =\sum_{k=1}^{n-1} s_{J, n-k} \psi_{J, k}-\psi_{J-1, n-1}, \quad n \geq 1,
\end{aligned}
$$


with the explicitly calculated convolution kernel:

$$
\begin{gathered}
s_{j, n}=\left[1-i \frac{R}{2}+\frac{\sigma_{j}}{2}\right] \delta_{n, 0}+\left[1+i \frac{R}{2}+\frac{\sigma_{j}}{2}\right] \delta_{n, 1}+\alpha_{j} e^{-i n \varphi_{j}} \frac{P_{n}\left(\mu_{j}\right)-P_{n-2}\left(\mu_{j}\right)}{2 n-1}, \\
\varphi_{j}=\arctan \frac{2 R\left(\sigma_{j}+2\right)}{R^{2}-4 \sigma_{j}-\sigma_{j}^{2}}, \quad \mu_{j}=\frac{R^{2}+4 \sigma_{j}+\sigma_{j}^{2}}{\sqrt{\left(R^{2}+\sigma_{j}^{2}\right)\left[R^{2}+\left(\sigma_{j}+4\right)^{2}\right]}} \\
\sigma_{j}=2 \Delta x^{2} V_{j}, \quad \alpha_{j}=\frac{i}{2} \sqrt[4]{\left(R^{2}+\sigma_{j}^{2}\right)\left[R^{2}+\left(\sigma_{j}+4\right)^{2}\right]} e^{i \varphi_{j} / 2}, \quad j=0, J
\end{gathered}
$$

Here $P_{n}$ denotes the Legendre polynomials $\left(P_{-1} \equiv P_{-2} \equiv 0\right)$, and $\delta_{n, k}$ is the Kronecker symbol.

In order to formulate the DTBC as in (1.10) it is necessary that the discrete initial condition vanishes at the two adjacent (spatial) grid points appearing in (1.10). Here, we chose to formulate the DTBC at the boundary of the computational interval and one grid point in the interior. Hence we have assumed that the initial condition satisfies $\psi_{0,0}=\psi_{1,0}=0$ and $\psi_{J-1,0}=\psi_{J, 0}=0$. However, without any change to our subsequent analysis one could also prescribe the DTBC at $j=-1,0$ and at $j=J, J+1$, resp.

The use of the formulas (1.17) for calculations permits us to avoid any boundary reflections and it renders the fully discrete scheme unconditionally stable (just like the underlying Crank-Nicolson scheme). However, the linearly in $t$ increasing numerical effort to evaluate the DTBCs can sharply raise the total computational costs. Note that we need to evaluate just one convolution of (1.17) at each time step (at the endpoint of the interval $\left.\left[0, t_{n}\right]\right)$. Since the other points of this convolution are not needed, using an FFT is not practical. A strategy to overcome this drawback will be the key issue of this paper.

The considered DTBCs (1.17) include the discrete convolution of the unknown function with a given kernel (1.18). Our approach for fast evaluation of this convolution consists of approximating the kernel by a finite sum of exponentials that decay with respect to time (cf. [29]): this will permit us to use recurrence formulas for the time stepping, see Section 4. Such kind of trick has been proposed in [44] for the continuous $\mathrm{TBC}$ in case of the $3 \mathrm{D}$ wave equation, and developed in $[1,2,45,46,16,22]$ for various hyperbolic problems.

Related results. As for the Schrödinger equation, we remark that a related approach was proposed by Bruneau and Di Menza $([13,17])$. There the authors consider the continuous TBC in Laplace space (for $V_{-}=0$ ):

$$
\hat{\psi}_{x}(0, s)=\sqrt{2} e^{-\frac{\pi}{4} i} \sqrt[+]{s} \hat{\psi}(0, s) ; \quad s=i \xi, \xi \in \mathbb{R}
$$

where $\sqrt[+]{ }$ denotes the branch of the square root with positive real part. Its symbol $\sqrt[+]{s}$ is represented by a rational function calculated with the help of a least-squares approximation on the imaginary axis. This approach gives decaying sums of exponentials for the convolution kernel but does not allow for a convergence analysis or error estimates of the resulting finite difference scheme (see Example 6.3 below).

The limit $\Delta x \rightarrow 0$ of the DTBCs (1.17) coincides with the temporally semidiscrete TBC of Schmidt and Deufhard [41] and of Lubich and Schädle, cf. [28, 38]. 
On the other hand, alternative derivations of the DTBC (1.17) could be done by applying the Mikusinsski operator approach, cf. [42], or the operational calculus, cf. [28], to the convolution-type BC of the spatially semi-discretized Schrödinger equation. A temporal semi-discretization of the Dirichlet-to-Neumann map for the Schrödinger equation on a circular domain was discussed in [38, 39].

In $[29,40]$ the continuous convolution kernel of the TBC for the Schrödinger equation and its spatial semi-discretization is approximated by sums of exponentials $e^{\lambda_{j} t}$ including terms with $\operatorname{Re}\left(\lambda_{j}\right)>0$, i.e. not decaying with respect to time. However, the authors propose an algorithm allowing to maintain a uniform relative error of the convolution kernel, but it requires to introduce new sums of exponentials to handle time intervals of an exponentially growing length.

Notice that all of the key ideas that we use for the Schrödinger equation here can easily be generalized to other types of partial differential equations like parabolic problems where the DTBCs have a similar form: In [19] DTBCs for the $\theta$-scheme for linear parabolic equations were derived. Also, other interior discretization scheme for the Schrödinger equation could be used (e.g. a Numerov-type discretization [32]).

The paper is organized as follows. In $\S 2$ we discuss the numerical computation of the convolution coefficients (via the inverse $Z$-transform), approximate them by a discrete sum of exponentials in $\S 3$, and present an efficient recursion for evaluating these approximate DTBCs in $\S 4$. In $\S 5$ we analyze the stability of the resulting initial-boundary-value scheme, and derive error estimates for the resulting Schrödinger solution in $\S 6$. Finally, the numerical examples of $\S 7$ illustrate the efficiency of the proposed method.

\section{Calculation of convolution coefficients}

The convolution coefficients $s_{0, n}, s_{J, n}$ appearing in the DTBC (1.17) can be calculated by the explicit formulae (1.18) as well as by 3 -term-recurrence formulae, see [20]. Let us describe another, more general method based on a numerical calculation of the inverse $Z$-transform, see [18]. According to the definition of the $Z$-transform, see (1.11), we need to calculate the coefficients of the Laurent series (1.16). By using the Cauchy integral representation on the circle with a radius $\rho>1$ one obtains

$$
s_{n}=\frac{\rho^{n}}{2 \pi} \int_{0}^{2 \pi} \hat{s}\left(\rho e^{i \varphi}\right) e^{i n \varphi} d \varphi, \quad n \in \mathbb{N}_{0},
$$

(for simplicity of notation we suppress here the index $j$ ).

The numerical approximation of (2.1) is made by the standard $N$-point summation rule:

$$
s_{n} \approx s_{n}^{(N)}=\frac{\rho^{n}}{N} \sum_{k=0}^{N-1} \hat{s}\left(\rho e^{i \varphi_{k}}\right) e^{i n \varphi_{k}}, \quad n=0,1, \ldots, N-1,
$$

where $\varphi_{k}=2 \pi k / N$. It is easy to show that the sum (2.2) yields the first $N$ values of $s_{n}$ with the accuracy

$$
s_{n}^{(N)}=s_{n}+\mathcal{O}\left(\rho^{-N}\right), \quad n=0,1, \ldots, N-1,
$$

provided that the sequence $\left\{s_{n}\right\}$ is bounded: Indeed, using the Laurent series (1.16) with $z=\rho e^{i \varphi}$ and taking into account the orthogonality property

$$
\sum_{k=0}^{N-1} e^{i n \varphi_{k}} e^{-i m \varphi_{k}}=N \delta_{n,(m \bmod N)}
$$


we obtain

$$
s_{n}^{(N)}=\frac{\rho^{n}}{N} \sum_{k=0}^{N-1} e^{i n \varphi_{k}}\left(\sum_{m=0}^{\infty} s_{m} \rho^{-m} e^{-i m \varphi_{k}}\right)=s_{n}+\rho^{-N} s_{n+N}+\rho^{-2 N} s_{n+2 N}+\ldots .
$$

Hence

$$
\left|s_{n}^{(N)}-s_{n}\right| \leq \frac{\rho^{-N}}{1-\rho^{-N}} \max _{k \geq N}\left|s_{k}\right|, \quad n=0,1, \ldots
$$

Estimate (2.4) means that we can obtain very accurate coefficients $s_{n}^{(N)}$ if we take an appropriate value of $\rho$ greater than 1: supposing that $\max _{k \geq N}\left|s_{k}\right|<1-\rho^{-N}$, we find from (2.4) that the accuracy

$$
\varepsilon:=\max _{n \geq 0}\left|s_{n}^{(N)}-s_{n}\right|
$$

can be guaranteed by the choice $\rho \geq \varepsilon^{-\frac{1}{N}}$; for example if $\varepsilon=10^{-6}, N=50$ then $\rho \geq 1.32$.

Note that estimates of kind (2.4) are well-known, see e.g. [27], [30], and references therein.

REMARK 1. By using the asymptotic behaviour $\left|s_{n}\right| \leq C n^{-3 / 2}$, see (1.15), we get the sharper estimate

$$
\rho \geq\left(1+\frac{C}{\varepsilon} N^{-3 / 2}\right)^{1 / N} .
$$

We shall now describe some numerical aspects of evaluating the inverse $Z$-transfo$\mathrm{rm}$. Our tests show that the numerical inverse $Z$-transformation based on (2.2) is very sensitive to the mantissa length (parameter Digits in Figure 2.1), i.e. to the round-off error. For large numbers $N$, e.g. $N>100$, the usual accuracy (e.g. Digits=15) could be insufficient.

For instance, Figure 2.1 shows the relative error (discrete $L^{2}$-norm) of $s_{n}^{(N)}$ in dependence of $\rho \geq 1$ using $N=256$ integration points. The curves look like a 'hook': for each fixed mantissa length the error first drops with increasing $\rho$ in accordance with the error estimate (2.4); for larger $\rho$ it then grows because of exponential amplification of the round-off errors due to the factor $\rho^{N}$ in (2.2). Note that for different values of Digits the decreasing part of the error curve (corresponding to the approximation error) overlaps exactly. For further discussions of the numerical inverse $Z$-transform, we refer the reader to [27] where, in particular, the question of choosing the computational radius $\rho$ in (2.2) is considered, and to [49] where a detailed numerical investigation of this situation is given.

\section{Approximation of convolution coefficients by sums of exponentials}

In order to derive a fast numerical method to calculate the discrete convolutions in (1.17) (for the algorithm cf. Section 4), we will approximate the coefficients $s_{n}$ by the following ansatz (sum of exponentials):

$$
s_{n} \approx \tilde{s}_{n}:= \begin{cases}s_{n}, & n=0, \ldots, \nu-1, \\ \sum_{l=1}^{L} b_{l} q_{l}^{-n}, & n=\nu, \nu+1, \ldots,\end{cases}
$$




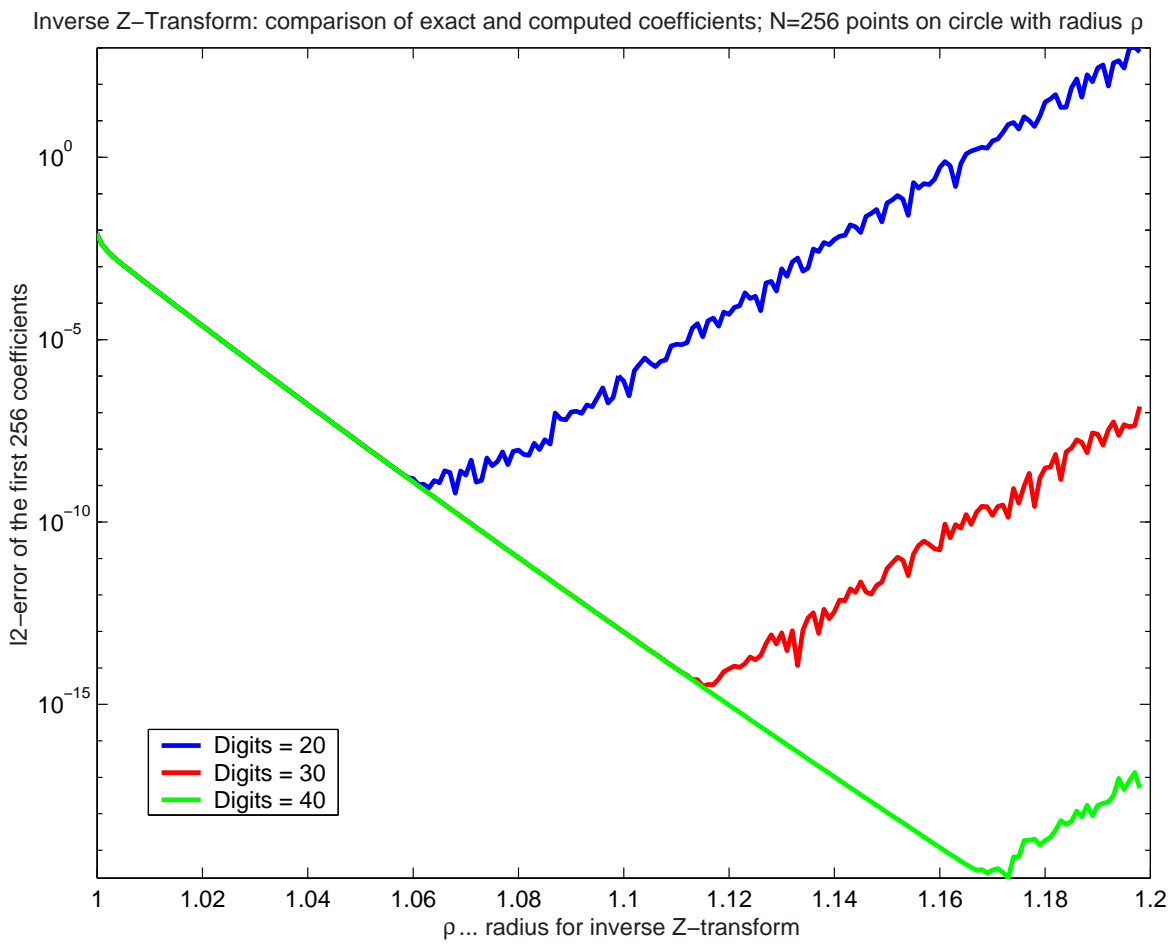

FIG. 2.1. Relative error of the numerical inverse $Z$-transform of $\hat{s}$ depending on $\rho$ (the radius of the integration circle) for different values of Digits (the mantissa length). The three curves overlap exactly on the decaying branch, and their increasing branches - corresponding to the amplified roundoff error - are 10 orders of magnitude apart. Here $\Delta x=1 / 160, \Delta t=2 \cdot 10^{-5}, N=256$.

where $L \in \mathbb{N}, \nu \geq 0$ are fixed numbers. Evidently, the approximation properties of $\tilde{s}_{n}$ depend on $L, \nu$, and the corresponding set $\left\{b_{l}, q_{l}\right\}$. Thus, the choice of an (in some sense) optimal such approximation is a difficult nonlinear problem. Below we propose a deterministic method of finding $\left\{b_{l}, q_{l}\right\}$ for fixed $L$ and $\nu$.

The "split" definition of $\left\{\tilde{s}_{n}\right\}$ in (3.1) is motivated by the fact that the implementation of the DTBCs (1.17) involves a convolution sum with $k$ ranging only from 1 to $k=n-1$. Since the first coefficient $s_{0}$ does not appear in this convolution, it makes no sense to include it in our sum-of-exponential approximation, which aims at simplifying the evaluation of the convolution. Hence, one may choose $\nu=1$ in (3.1). The "special form" of $s_{0}$ and $s_{1}$ in definition (1.18) suggests even to exclude $s_{1}$ from this approximation and to choose $\nu=2$ in (3.1); note that $\alpha^{-1} e^{i n \varphi} s_{n} \in \mathbb{R}$ for $n \geq 2$. We use this choice in our numerical implementation.

Also, there is an additional motivation for choosing $\nu=2$ : With the choice $\nu=0$ (or $\nu=1$ ) we typically obtain two (or, resp., one) coefficient pairs $\left(b_{l}, q_{l}\right)$ of big magnitude. These "outlier" values reflect the different nature of the first two coefficients. Including them into our discrete sum-of-exponential would then yield less accurate approximation results.

Let us fix $L$ and $\nu$ in (3.1), and consider the formal power series:

$$
f(x):=s_{\nu}+s_{\nu+1} x+s_{\nu+2} x^{2}+\ldots
$$


for $|x| \leq 1$. If there exists the $[L-1 \mid L]$ Padé approximation

$$
\tilde{f}(x):=\frac{P_{L-1}(x)}{Q_{L}(x)}
$$

of (3.2), then its Taylor series

$$
\tilde{f}(x)=\tilde{s}_{\nu}+\tilde{s}_{\nu+1} x+\tilde{s}_{\nu+2} x^{2}+\ldots
$$

satisfies the conditions

$$
\tilde{s}_{n}=s_{n}, \quad n=\nu, \nu+1, \ldots, 2 L+\nu-1,
$$

according to the definition of the Padé approximation rule.

TheOrem 3.1. Assume $Q_{L}(x)$ have $L$ simple roots $q_{l}$ with $\left|q_{l}\right|>1, \quad l=1, \ldots, L$. Then

$$
\tilde{s}_{n}=\sum_{l=1}^{L} b_{l} q_{l}^{-n}, \quad n=\nu, \nu+1, \ldots,
$$

where

$$
b_{l}:=-\frac{P_{L-1}\left(q_{l}\right)}{Q_{L}^{\prime}\left(q_{l}\right)} q_{l}^{\nu-1} \neq 0, \quad l=1, \ldots, L .
$$

Proof. We use the following known representation of the rational function (3.3):

$$
\frac{P_{L-1}(x)}{Q_{L}(x)}=\sum_{l=1}^{L} \frac{b_{l} q_{l}^{1-\nu}}{q_{l}-x}
$$

in terms of $\left\{b_{l}, q_{l}\right\}$ defined in the formulation of this theorem, see e.g. [16]. Substituting the identity

$$
\frac{1}{q_{l}-x}=q_{l}^{-1} \sum_{n=0}^{\infty}\left(\frac{x}{q_{l}}\right)^{n}
$$

(with $|x|<\left|q_{l}\right|$ ) in (3.8), we obtain (3.6) by comparing equations (3.3) and (3.4).

REMARK 2. All our practical calculations confirm that the assumption on $Q_{L}(x)$ in Theorem 3.1 holds for any desired L, although we cannot prove this.

It follows from (3.5) and (3.6) that the set $\left\{b_{l}, q_{l}\right\}$ defined in Theorem 3.1 can be used in (3.1) at least for $n=\nu, \nu+1, \ldots, 2 L+\nu-1$. The main question now is: is it possible to use these $\left\{b_{l}, q_{l}\right\}$ also for $n>2 L+\nu-1$ ? In other words, what quality of approximation

$$
\tilde{s}_{n} \approx s_{n}, \quad n>2 L+\nu-1
$$

can we expect?

Having in mind to outline our approach for a general case (not only for the specific $s_{n}$ defined in (1.18)) we will use (a slight generalization of) the Baker-Gammel-Wills conjecture [8] concerning the existence of convergent subsequences of diagonal Padé approximants. 
Conjecture (Generalization of Baker-Gammel-Wills, [8]) 1. Let $p(x)$ be a power series representing a function which is meromorphic in $|x|<1$ and continuous on $|x| \leq 1$ (except at the poles inside the unit circle). Then, at least a subsequence of the $[L \mid L]$ Padé approximants converges uniformly to $p(x)($ as $L \rightarrow \infty)$ in the domain formed by removing from the closed unit disk small open circles around the poles of $p$.

Remark 3. The study of $[L-1 \mid L]$ Padé approximants can be reduced to the study of the $[L \mid L]$ case by simply considering Padé approximants to $g(x)=x p(x)$.

Coming back to our function $f(x)$ with $s_{n}$ defined in (1.18), we note that it is analytic on $|x|<1$ and continuous on $|x| \leq 1$. This follows from the analogous properties of $\hat{s}(z)$ for $|z| \geq 1$.

Theorem 3.2. If the Conjecture holds, we then have:

(i) at least a subsequence of the $[L-1 \mid L]$ (bounded) Padé approximants to (3.2) converges uniformly on the disk $|x| \leq 1$, as $L \rightarrow \infty$, and we have

$$
\begin{aligned}
\hat{\tilde{s}}^{(L)}(z) & \stackrel{L \rightarrow \infty}{\longrightarrow} \hat{s}(z), \text { uniformly for }|z| \geq 1 ; \\
\left\{\tilde{s}_{n}^{(L)}\right\} & \stackrel{L \rightarrow \infty}{\longrightarrow}\left\{s_{n}\right\} \text { in } \ell^{2}\left(\mathbb{N}_{0}\right) .
\end{aligned}
$$

Here, $\tilde{s}_{n}^{(L)}$ denotes the coefficients (3.6) obtained for a fixed $L$.

(ii) all roots of $Q_{L}(x)$ (of the above subsequence) have absolute values greater than 1 ;

(iii) $\left|s_{n}-\tilde{s}_{n}\right|=\mathcal{O}\left(n^{-\frac{3}{2}}\right)$, L fixed, $n \rightarrow \infty$.

Proof. The power series (3.2) with $s_{n}$ defined in (1.18) is analytic on $|x|<1$ and continuous for $|x| \leq 1$. Therefore we obtain the first part of conclusion $(i)$ directly from the conjecture. With $z=1 / x$ the convergence of $\tilde{f}^{(L)}(x),|x| \leq 1$ immediately implies the convergence of

$$
\hat{\tilde{s}}^{(L)}(z)=\sum_{n=0}^{\nu-1} s_{n} z^{-n}+\tilde{f}^{(L)}\left(z^{-1}\right) z^{-\nu},
$$

and hence the convergence of its inverse $Z$-transform, the convolution coefficients $\tilde{s}_{n}^{(L)}$. The property (ii) follows from $(i)$ since any root $q_{l}$ of $Q_{L}(x)$ with $\left|q_{l}\right| \leq 1$ would violate (i). Finally, (iii) follows from (ii) and (3.6) (or its modifications for multiple roots), which shows that $\left|\tilde{s}_{n}\right| \rightarrow 0$ exponentially, and from (1.15).

REMARK 4. We remark that the assumption (from Theorem 3.1) on the roots of $Q_{L}(x)$ to be simple is not essential for Theorem 3.2. For multiple roots one only has to reformulate Theorem 3.1.

The above analysis permits us to give the following description of the approximation to the convolution coefficients (1.18) by the representation (3.1) if we use a $[L-1 \mid L]$ Padé approximant to (3.2): the first $2 L+\nu-1$ coefficients are reproduced exactly, see (3.5); however, the asymptotic behaviour of $s_{n}$ and $\tilde{s}_{n}($ as $n \rightarrow \infty)$ differs strongly (algebraic versus exponential decay). A typical graph of $\left|s_{n}-\tilde{s}_{n}\right|$ versus $n$ for $L=20$ is shown in Figure 3.1.

So far we discussed how to calculate and to approximate the DTBCs for one fixed discretization of the 1D-Schrödinger equation. However, a nice property of the considered approach consists of the following: once the approximate convolution 


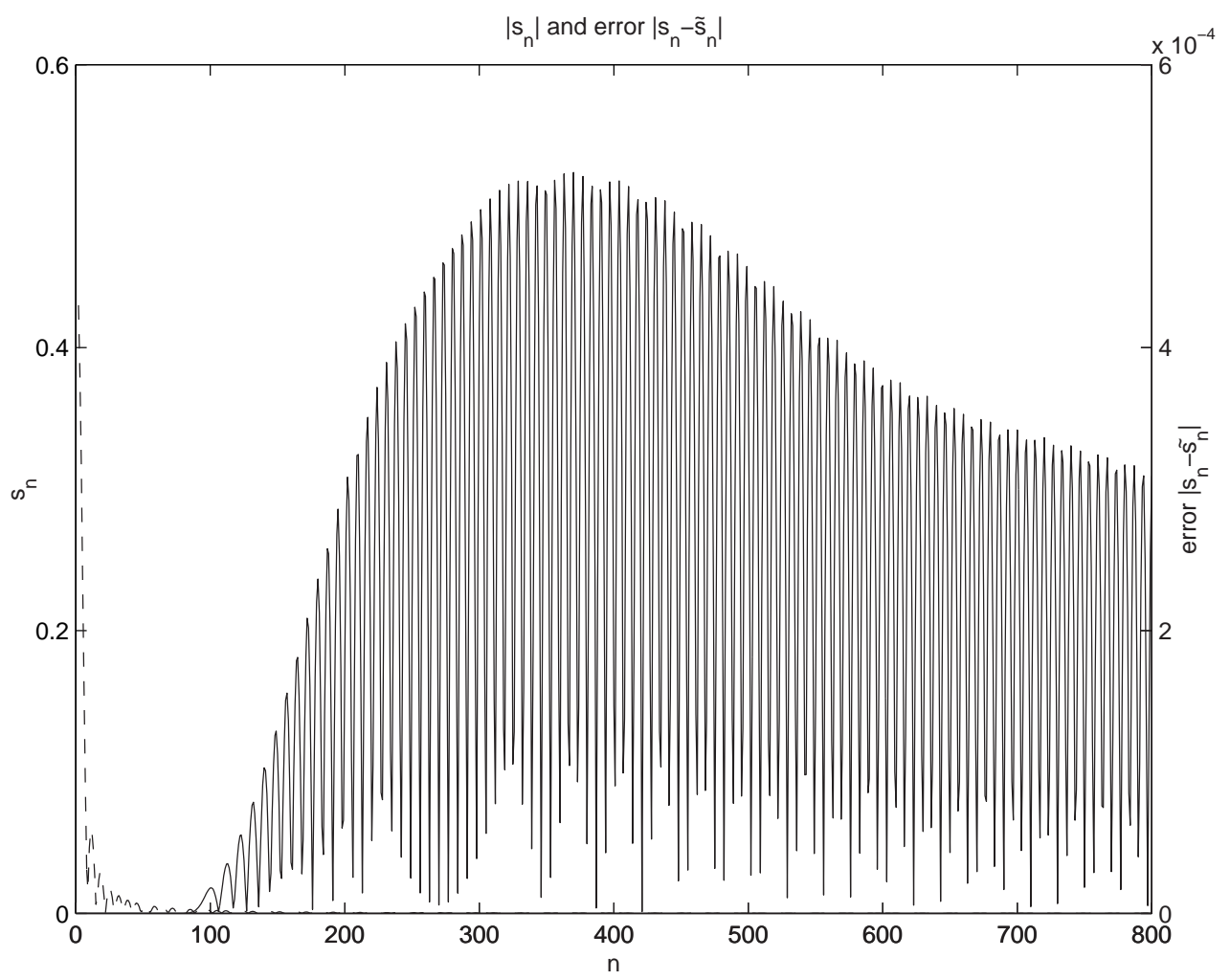

FIG. 3.1. Convolution coefficients $s_{n}$ (left axis, dashed line) and error $\left|s_{n}-\tilde{s}_{n}\right|$ of the convolution coefficients (right axis); $\Delta x=1 / 160, \Delta t=2 \cdot 10^{-5}, V=0(L=20)$

coefficients $\left\{\tilde{s}_{n}\right\}$ are calculated for particular discretization parameters $\{\Delta x, \Delta t, V\}$, it is easy to transform them into appropriate coefficients for any other discretization. We shall confine this discussion to the case $\nu=2$ :

Transformation Rule 3.1. For $\nu=2$, let the rational function

$$
\hat{\tilde{s}}(z)=s_{0}+\frac{s_{1}}{z}+\sum_{l=1}^{L} \frac{b_{l}}{q_{l} z-1} \frac{1}{q_{l} z}
$$

be the $Z$-transform of the convolution kernel $\left\{\tilde{s}_{n}\right\}_{n=0}^{\infty}$ from (3.1), where $\left\{\tilde{s}_{n}\right\}$ is assumed to be an approximation to a DTBC for the equation (1.8) with a given set $\{\Delta x$, $\Delta t, V\}$.

Then, for another set $\left\{\Delta x_{\star}, \Delta t_{\star}, V_{\star}\right\}$, one can take the approximation

$$
\hat{\tilde{s}}^{\star}(z):=s_{0}^{\star}+\frac{s_{1}^{\star}}{z}+\sum_{l=1}^{L} \frac{b_{l}^{\star}}{q_{l}^{\star} z-1} \frac{1}{q_{l}^{\star} z},
$$


where

$$
\begin{aligned}
q_{l}^{\star} & :=\frac{q_{l} \bar{a}-\bar{b}}{a-q_{l} b}, \\
b_{l}^{\star} & :=b_{l} q_{l} \frac{a \bar{a}-b \bar{b}}{\left(a-q_{l} b\right)\left(q_{l} \bar{a}-\bar{b}\right)} \frac{1+q_{l}^{\star}}{1+q_{l}}, \\
a & :=2 \frac{\Delta x^{2}}{\Delta t}+2 \frac{\Delta x_{\star}^{2}}{\Delta t_{\star}}+i\left(\Delta x^{2} V-\Delta x_{\star}^{2} V_{\star}\right), \\
b & :=2 \frac{\Delta x^{2}}{\Delta t}-2 \frac{\Delta x_{\star}^{2}}{\Delta t_{\star}}-i\left(\Delta x^{2} V-\Delta x_{\star}^{2} V_{\star}\right),
\end{aligned}
$$

and $s_{0}^{\star}, s_{1}^{\star}$ are the exact convolution coefficients for the parameters $\left\{\Delta x_{\star}, \Delta t_{\star}, V_{\star}\right\}$ as given by (1.18).

Derivation. This transformation rule is based on the observation that the exact $Z$ transformed boundary kernel $\hat{\ell}(z)=\frac{z}{z+1} \hat{s}(z)$ depends on the parameters $\Delta x, \Delta t$, and $V$ only via the variable

$$
\zeta=2 \frac{\Delta x^{2}}{\Delta t} \frac{z-1}{z+1}+i \Delta x^{2} V
$$

cf. (1.12).

Making the assumption that also the approximate transformed boundary kernels

$$
\begin{gathered}
\hat{\tilde{\ell}}(z):=\frac{z}{z+1} \hat{\tilde{s}}(z), \\
\hat{\tilde{\ell}^{\star}}\left(z^{\star}\right):=\frac{z^{\star}}{z^{\star}+1} \hat{\tilde{s}}^{\star}\left(z^{\star}\right)
\end{gathered}
$$

only depend on $\zeta$ yields a transformation between two sets of parameters (we shall elaborate on this choice in $\S 5$, cf. (5.12)). By equating the functions $\zeta$ and $\zeta^{\star}$ corresponding to the sets $\{\Delta x, \Delta t, V\}$ and $\left\{\Delta x_{\star}, \Delta t_{\star}, V_{\star}\right\}$, respectively, we obtain the map

$$
z^{\star}=\frac{a z-b}{\bar{a}-\bar{b} z}
$$

with $a, b$ defined above.

With (3.18) and (3.19) the obvious transformation for $\hat{\tilde{\ell}}$, i.e.

$$
\hat{\tilde{\ell}}\left(z^{\star}\right) \equiv \hat{\tilde{\ell}}\left(z\left(z^{\star}\right)\right) \quad \text { with } z\left(z^{\star}\right)=\frac{\bar{a} z^{\star}+b}{a+\bar{b} z^{\star}}
$$

translates into a transformation for $\hat{\tilde{s}}$ :

$$
\hat{\tilde{s}}^{\star}\left(z^{\star}\right)=\frac{z^{\star}+1}{z^{\star}} \hat{\tilde{\ell}}\left(z^{\star}\right)=\frac{z^{\star}+1}{z^{\star}} \hat{\tilde{\ell}}\left(z\left(z^{\star}\right)\right)=\frac{z^{\star}+1}{z^{\star}} \frac{z\left(z^{\star}\right)}{z\left(z^{\star}\right)+1} \hat{\tilde{s}}\left(z\left(z^{\star}\right)\right) .
$$

Using $z\left(z^{\star}\right)$ in (3.11) a lengthy but straight forward calculation yields

$$
\hat{\tilde{s}}^{\star}\left(z^{\star}\right)=c_{0}^{\star}+\frac{c_{1}^{\star}}{z^{\star}}+\sum_{l=1}^{L} \frac{b_{l}^{\star}}{q_{l}^{\star} z^{\star}-1} \frac{1}{q_{l}^{\star} z^{\star}},
$$


where $q_{l}^{\star}$ and $b_{l}^{\star}$ are defined, respectively, in (3.13) and (3.14). The constants $c_{0}^{\star}$ and $c_{1}^{\star}$ depend on $s_{0}, s_{1}, b_{l}$, and $q_{l}$, and, in general, they do not coincide with the exact values $s_{0}^{\star}$ and $s_{1}^{\star}$. For our purposes, however, this does not matter: Here, we are only interested in the transformation of the exponential approximation, since only this part enters the convolution (1.17) (cf. $\S 4$ below). Otherwise,the calculation of $\left\{\tilde{s}_{n}\right\}$ would require to use the Padé -algorithm discussed in Theorem 3.1.

(3.22) shows that the transformation (3.21) preserves the structure of our approximate convolution coefficients $\left\{\tilde{s}_{n}\right\}$ as a sum of discrete exponentials for $n \geq \nu=2$ (cf. (3.1)).

It remains to note that due to $|b|<|a|$, the bilinear function (3.20) maps the unit disc onto itself (see [24], e.g.). Therefore, for $\left|q_{l}\right|>1$ the map (3.13) gives also $\left|q_{l}^{\star}\right|>1$. Hence, $\left\{\tilde{s}_{n}^{\star}\right\}$ also contains only decaying exponentials.

The importance of this Transformation rule is twofold: While the Padé -algorithm provides a method to calculate approximate convolution coefficients $\tilde{s}_{n}$ for fixed parameters $\{\Delta x, \Delta t, V\}$, the Transformation rule yields the natural link (via the assumption (3.17)-(3.19)) between different parameter sets $\left\{\Delta x_{\star}, \Delta t_{\star}, V_{\star}\right\}$ (and $L$ fixed). This will be an important basis for the stability analysis (as $\Delta x_{\star}, \Delta t_{\star} \rightarrow 0$ ) in Section 5 .

Moreover, it allows to extend the convergence result Theorem 3.2(i) on the convolution coefficients $\tilde{s}_{n}^{(L)}$ as $L \rightarrow \infty$, uniformly in $\Delta x_{\star}, \Delta t_{\star}$ :

Theorem 3.3. If the Conjecture holds, we then have:

Let the coefficients $\left\{\tilde{s}_{n}^{(L)}\right\}$ be obtained for the parameters $\{\Delta x, \Delta t, V\}$ via the above Padé-method for $L \in \mathbb{N}$.

Then the coefficients $\left\{\tilde{s}_{n}^{\star}{ }^{(L)}\right\}$ for $\Delta x_{\star}, \Delta t_{\star}$, and $V_{\star}=V$, obtained via the transformation (3.21)-(3.22) satisfy

$$
\left\|\tilde{s}_{n}^{\star(L)}-s_{n}^{\star}\right\|_{2} \leq C\left\|\tilde{s}_{n}^{(L)}-s_{n}\right\|_{2},
$$

where the constant $C$ is uniform for $\Delta x_{\star} \leq C_{1}, \Delta x_{\star}^{2} / \Delta t_{\star} \leq C_{2}$. And (at least a subsequence of) the error $\left\|\tilde{s}_{n}^{(L)}-s_{n}\right\|_{2} \stackrel{L \rightarrow \infty}{\longrightarrow} 0$ by Theorem 3.2(i).

Proof. Using (3.21) for $\hat{\tilde{s}}^{\star(L)}$ and $\hat{s}^{\star}$ we have

$$
\left|\hat{\tilde{s}}^{\star(L)}\left(z^{\star}\right)-\hat{s}^{\star}\left(z^{\star}\right)\right|=\frac{\Delta t}{4 \Delta x^{2}}\left|w\left(z^{\star}\right)\right| \cdot\left|\hat{\tilde{s}}^{(L)}\left(z\left(z^{\star}\right)\right)-\hat{s}\left(z\left(z^{\star}\right)\right)\right| .
$$

Here, the bilinear function $w\left(z^{\star}\right)=\left(\bar{a} z^{\star}+b\right) / z^{\star}$ maps the unit circle $z^{\star}=e^{i \varphi}$ onto the circle that is determined by the two "diagonal points"

$$
\begin{aligned}
w(1) & =4 \frac{\Delta x^{2}}{\Delta t}-2 i V\left(\Delta x^{2}-\Delta x_{\star}^{2}\right), \\
w(-1) & =4 \frac{\Delta x_{\star}^{2}}{\Delta t_{\star}} .
\end{aligned}
$$

Hence, $w\left(e^{i \varphi}\right)$ is uniformly bounded with respect to $0 \leq \varphi \leq 2 \pi, \Delta x_{\star} \leq C_{1}$, $\Delta x_{\star}^{2} / \Delta t_{\star} \leq C_{2}$. Since $z\left(e^{i \varphi}\right)$ is a self-map of the unit circle, the assertion (3.23) follows from Plancherel's Theorem for $Z$-transforms.

We remark that the assumption $V_{\star}=V$ in Theorem 3.3 is only due to the fact that we are interested here in the stability of one fixed model w.r.t. to the discretization parameters $\Delta x, \Delta t, L$. In fact, estimate (3.23) could be extended to $V_{\star} \neq V$. 
We now return to the discrete 2D-Schrödinger equation, which was decomposed into the modes $\psi^{m}, m=1, \ldots, K-1$ in (1.7). Provided an exponential approximation of DTBCs for a single mode is known, we can use formulae (3.13), (3.14) for a rapid calculation of approximate DTBCs for the remaining Fourier modes by taking the corresponding values $V_{j}^{m}$.

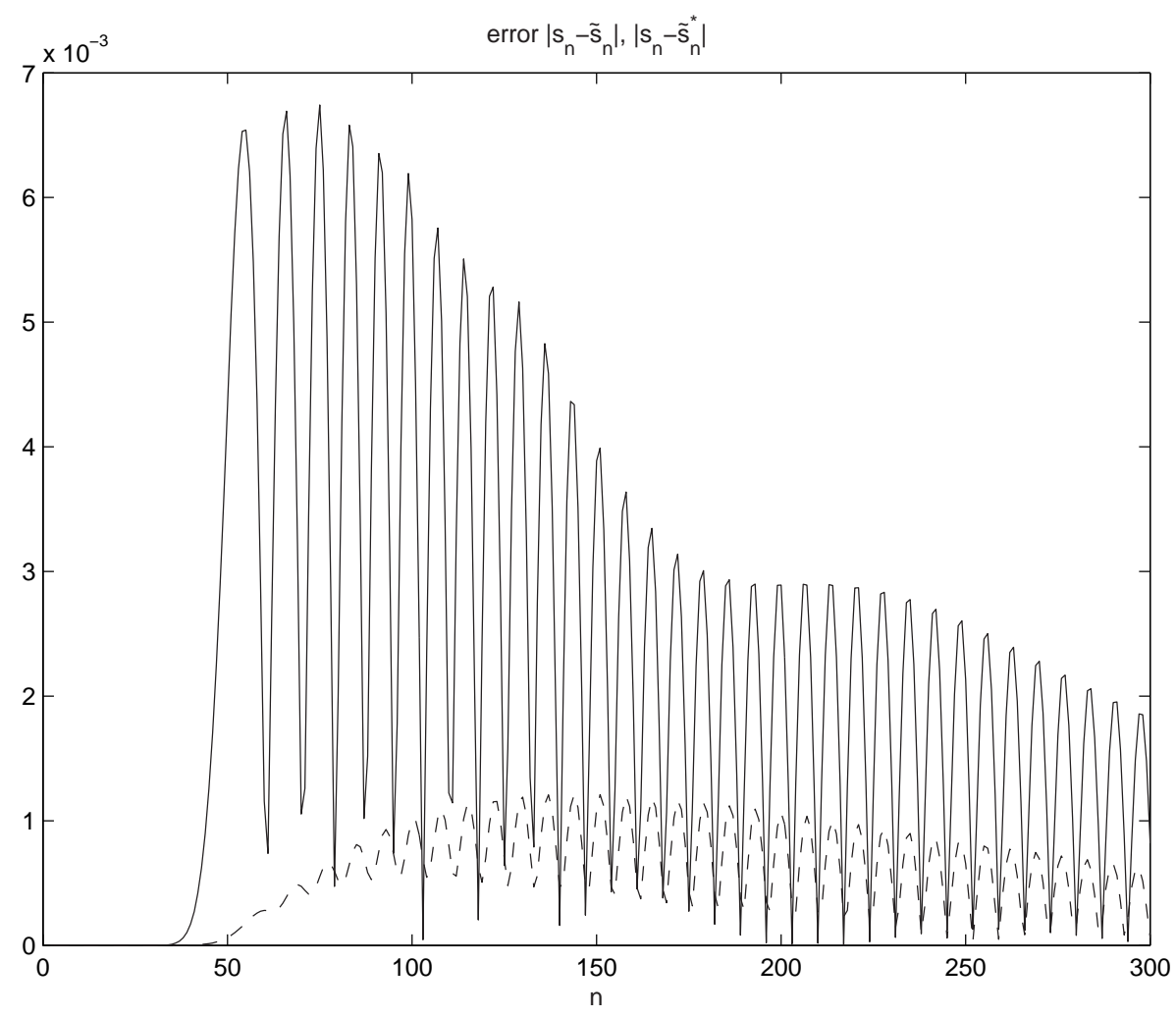

FIG. 3.2. Approximation error of the approximate convolution coefficients for $\nu=2, \Delta x=$ 1/160, $\Delta t=2 \cdot 10^{-5}, V=4500$ : The error of $\tilde{s}_{n}^{*}(--)$ obtained from the transformation rule and the error of $\tilde{s}_{n}$ (-) obtained from a direct Padé approximation of the exact coefficients $s_{n}$.

Example 3.1. (Transformation rule) We present a numerical example for applying the above transformation rule. For $L=10$ we calculated the coefficients $\left\{b_{l}, q_{l}\right\}$ with the parameters $\Delta x=1, \Delta t=1, V=0$ (cf. Appendix A) and then used the Transformation 3.1 to calculate the coefficients $\left\{b_{l}^{*}, q_{l}^{*}\right\}$ for the parameters $\Delta x_{*}=1 / 160, \Delta t_{*}=2 \cdot 10^{-5}, V_{*}=4500$.

Figure 3.2 shows that the resulting convolution coefficients $\tilde{s}_{n}^{*}$ are in this example even better approximations to the exact coefficients $s_{n}$ than the coefficients $\tilde{s}_{n}$, which are obtained directly from the Padé algorithm discussed in Theorem 3.1. Hence, the numerical solution of the corresponding Schrödinger equation is also more accurate (cf. Example 7.2, Figure 7.6). 


\section{Fast evaluation of the discrete convolution with an "exponential" kernel}

Let us consider the approximation (3.1) of the discrete convolution kernel appearing in the DTBC (1.17). With these "exponential" coefficients the convolution

$$
C^{(n)}(u):=\sum_{k=1}^{n-\nu} u_{k} \tilde{s}_{n-k}, \quad \tilde{s}_{n}=\sum_{l=1}^{L} b_{l} q_{l}^{-n}, \quad\left|q_{l}\right|>1,
$$

of a discrete function $u_{k}, k=1,2, \ldots$, can be calculated by recurrence formulae, cf. [44]. Note that similar recursive convolution algorithms are successfully used in other applications as well, see [12] and references therein. This will reduce the numerical effort drastically (cf. Figure 7.7 in Example 7.2).

THEOREM 4.1. The function $C^{(n)}(u)$ from (4.1) for $n \geq \nu+1$ is represented by

$$
C^{(n)}(u)=\sum_{l=1}^{L} C_{l}^{(n)}(u)
$$

where

$$
\begin{aligned}
& C_{l}^{(n)}(u)=q_{l}^{-1} C_{l}^{(n-1)}(u)+b_{l} q_{l}^{-\nu} u_{n-\nu}, \quad n=\nu+1, \nu+2, \ldots \\
& C_{l}^{(\nu)}(u) \equiv 0 .
\end{aligned}
$$

Proof. A straightforward calculation yields:

$$
C^{(n)}(u)=\sum_{k=1}^{n-\nu} u_{k} \sum_{l=1}^{L} b_{l} q_{l}^{-(n-k)}=\sum_{l=1}^{L} C_{l}^{(n)}(u),
$$

with

$$
C_{l}^{(n)}(u):=\sum_{k=1}^{n-\nu} b_{l} q_{l}^{-(n-k)} u_{k}
$$

And for each $C_{l}^{(n)}(u)$ we have the recursion:

$$
C_{l}^{(n)}(u)=q_{l}^{-1} \sum_{k=1}^{n-\nu-1} b_{l} q_{l}^{-(n-1-k)} u_{k}+b_{l} q_{l}^{-\nu} u_{n-\nu}=q_{l}^{-1} C_{l}^{(n-1)}(u)+b_{l} q_{l}^{-\nu} u_{n-\nu}
$$

with

$$
C_{l}^{(\nu)}(u) \equiv 0
$$

Finishing the algorithmic part of this study let us summarize the steps of the proposed method to evaluate approximate DTBCs:

Step 1: Prescribe $L, \nu$ (e.g. $\nu=2$ ) in (3.1), take $\Delta x=\Delta t=1, \rho \geq 1, N \geq 2 L+1$, and calculate by (1.18) or (2.2) the coefficients $s_{n}^{(N)}, n=\nu, \nu+1, \cdots, 2 L+\nu-1$. 
Step 2: Use the $[L-1 \mid L]$-Padé algorithm for the series (3.4) with $\tilde{s}_{n}:=s_{n}^{(N)}, n=$ $\nu, \nu+1, \cdots, 2 L+\nu-1$ in order to find $\left\{b_{l}, q_{l}\right\}$ for (3.1) in accordance with Theorem 3.1.

The Steps 1 and 2 are made once and for all; see Appendix A with the table of coefficients for $L=5,10$.

Step 3: For given $\left\{\Delta x_{\star}, \Delta t_{\star}, V_{\star}\right\}$ use formulae (3.13)-(3.16) with $\{\Delta x=1, \Delta t=$ $1, V=0\}$ and $\left\{b_{l}, q_{l}\right\}$ from Step 2 for the calculation of $\left\{b_{l}^{\star}, q_{l}^{\star}\right\}$.

Step 4: Implement the recurrence formulae (4.2), (4.3) to calculate approximate convolutions in (1.17). The coefficients $s_{0}^{*}, s_{1}^{*}, \ldots, s_{\nu-1}^{*}$ have to be calculated by (1.18) or (2.2).

\section{Stability analysis of the numerical scheme}

In this section we shall give a stability analysis (as $\Delta x, \Delta t \rightarrow 0$ ) of our numerical scheme for the Schrödinger equation (1.8) along with BCs of convolution form like (1.17). Usually, these BCs will be exactly or approximately transparent (with $L$, the number of exponential terms fixed), depending on the chosen convolution kernel.

$\mathbf{L}^{2}$-a-priori estimate of continuous solution. To illustrate our subsequent calculations for the discrete case we shall first give an a-priori estimate for a continuous IBVP for the Schrödinger equation:

$$
\left\{\begin{aligned}
i \psi_{t} & =-\frac{1}{2} \psi_{x x}+V(x, t) \psi, & & 0<x<X, t>0 \\
\psi(x, 0) & =\psi^{I}(x), & & 0<x<X \\
\psi_{x}(0, t) & =\int_{0}^{t} f_{0}(t-\tau) \psi(0, \tau) d \tau, & & t>0 \\
\psi_{x}(X, t) & =\int_{0}^{t} f_{X}(t-\tau) \psi(X, \tau) d \tau, & & t>0
\end{aligned}\right.
$$

where $f_{0}(t), f_{X}(t)$ are given functions, and we shall assume $\psi^{I} \in L^{2}(0, X)$.

Alternatively, the two boundary conditions can be written in the Laplace transform space as

$$
\begin{aligned}
\hat{\psi}_{x}(0, s)=\hat{f}_{0}(s) \hat{\psi}(0, s), & \operatorname{Re} s \geq 0, \\
\hat{\psi}_{x}(X, s)=\hat{f}_{X}(s) \hat{\psi}(X, s), & \operatorname{Re} s \geq 0,
\end{aligned}
$$

where $\hat{f}_{0}(s)$ and $\hat{f}_{X}(s), s \in \mathbb{C}$ are the Laplace transforms of $f_{0}(t)$ and $f_{X}(t), t \geq 0$, respectively.

For the system (5.1) we have the following estimate:

Proposition 5.1. (Stability condition) Let the transformed boundary kernels $\hat{f}_{0}, \hat{f}_{X}$ satisfy for some $\alpha_{1} \in \mathbb{R}$ :

$$
\operatorname{Im} \hat{f}_{0}\left(\alpha_{1}+i \xi\right) \leq 0, \quad \operatorname{Im} \hat{f}_{X}\left(\alpha_{1}+i \xi\right) \geq 0, \quad \forall \xi \in \mathbb{R} .
$$

Then the solution of (5.1) satisfies the a-priori estimate

$$
\|\psi(., t)\|_{L^{2}(0, X)} \leq\left\|\psi^{I}\right\|_{L^{2}(0, X)} e^{\alpha_{1} t}, \quad t>0 .
$$

Proof. For smooth solutions $\psi$ this proposition is easily proved by using an energy estimate for the function $\phi(x, t):=\psi(x, t) e^{-\alpha_{1} t}$ and by using Plancherel's identity for 
Laplace transforms:

$$
\begin{aligned}
&\|\phi(., t)\|_{L^{2}(0, X)}^{2} \leq\left\|\psi^{I}\right\|_{L^{2}(0, X)}^{2}+\operatorname{Im} \int_{0}^{t}\left[\phi(X, \tau) \overline{\phi_{x}}(X, \tau)-\phi(0, \tau) \overline{\phi_{x}}(0, \tau)\right] d \tau \\
&=\left\|\psi^{I}\right\|_{L^{2}(0, X)}^{2}+\operatorname{Im} \int_{0}^{\infty}\left[\chi_{[0, t]}(\tau) \phi(X, \tau) \overline{\left\{\left(\chi_{[0, t]} \phi\right) * \tilde{f}_{X}\right\}}(X, \tau)\right.\left.\quad-\chi_{[0, t]}(\tau) \phi(0, \tau) \overline{\left\{\left(\chi_{[0, t]} \phi\right) * \tilde{f}_{0}\right\}}(0, \tau)\right] d \tau \\
&=\left\|\psi^{I}\right\|_{L^{2}(0, X)}^{2}-\frac{1}{2 \pi} \int_{\mathbb{R}}\left[\left.\widehat{\mid \chi_{[0, t]}}\right|^{2}(X, i \xi) \operatorname{Im} \hat{f}_{X}\left(\alpha_{1}+i \xi\right)\right. \\
&\left.\quad-\left|\widehat{\chi_{[0, t]} \phi}\right|^{2}(0, i \xi) \operatorname{Im} \hat{f}_{0}\left(\alpha_{1}+i \xi\right)\right] d \xi \\
& \leq\left\|\psi^{I}\right\|_{L^{2}(0, X)}^{2} .
\end{aligned}
$$

Here $\chi$ denotes the characteristic function, and we used the fact that

$$
\phi_{x}(\tau)=\left(\phi * \tilde{f}_{0}\right)(\tau)=\left(\left(\chi_{[0, t]} \phi\right) * \tilde{f}_{0}\right)(\tau), \quad \tau \leq t,
$$

with the notation $\hat{\tilde{f}}_{0}(s)=\hat{f}_{0}\left(\alpha_{1}+s\right)$ (and analogously for $\left.f_{X}\right)$.

We remark that the calculation in (5.5) is rigorous for $\psi(0,),. \psi(X,.) \in L_{l o c}^{2}\left(\mathbb{R}_{0}^{+}\right)$ and $f_{0}, f_{X} \in L_{l o c}^{1}\left(\mathbb{R}_{0}^{+}\right)^{1}$.

Example 5.1. (Stability of TBC) The functions

$$
\hat{f}_{0}^{\mathrm{TBC}}(s)=\sqrt{2} e^{-\frac{\pi}{4} i} \sqrt[+]{s+i V_{-}}, \quad \hat{f}_{X}^{\mathrm{TBC}}(s)=-\sqrt{2} e^{-\frac{\pi}{4} i} \sqrt[+]{s+i V_{+}}
$$

correspond to the TBCs (1.2), (1.3). An easy calculation shows that they satisfy the stability conditions (5.3) for all $\alpha_{1} \geq 0$.

However, the above regularity assumptions on $f_{0}^{\mathrm{TBC}}, f_{X}^{\mathrm{TBC}}$ are not satisfied here: With $V_{-}=0$ the left TBC (1.2) reads

$$
\psi_{x}(0, t)=\sqrt{2} e^{-\frac{\pi}{4} i} \frac{d^{\frac{1}{2}} \psi}{d t^{\frac{1}{2}}}(0, t)
$$

where $\frac{d^{\frac{1}{2}}}{d t^{\frac{1}{2}}}$ denotes the semiderivative (cf. $\S 7$ in [33]). For smooth functions $\psi(0,$.$) it$ can be rewritten as

$$
\psi_{x}(0, t)=\sqrt{\frac{2}{\pi}} e^{-\frac{\pi}{4} i}\left[\frac{1}{2} \int_{0}^{t} \frac{\psi(0, t)-\psi(0, \tau)}{(t-\tau)^{3 / 2}} d \tau+\frac{\psi(0, t)}{\sqrt{t}}\right] .
$$

A part of a singular (distributional) contribution at $t=0$, its convolution kernel (as appearing in (5.1)) is

$$
f_{0}^{\mathrm{TBC}}(t)=-(2 \pi)^{-\frac{1}{2}} e^{-\frac{\pi}{4} i} t^{-\frac{3}{2}}, \quad t>0 .
$$

Note that this is the same decay rate as in the discrete case (1.15).

\footnotetext{
${ }^{1}$ With $L_{l o c}^{p}$ we denote spaces of locally integrable functions.
} 
For the free Schödinger equation (i.e. for $V \equiv 0$ ) with the TBCs (1.2), (1.3) the stability estimate (5.4) with $\alpha_{1}=0$ was proved in Theorem 2.2 .2 of [17] for $\psi^{I} \in$ $H^{1}(0, X)$. That proof easily extends to the case with a potential $V \in L^{\infty}\left(\mathbb{R}_{t}^{+} ; W^{1, \infty}(\mathbb{R})\right)$ that is constant outside the computational domain.

Example 5.2. (Sufficient condition for stability of "cut-off TBC") For the kernel of an approximate BC we assume a cut-off for $t \geq T$ and define:

$$
f_{0}(t)=f_{0}^{\mathrm{TBC}}(t) H(T-t),
$$

with $H(t)$ being the Heaviside function and $f_{0}^{\mathrm{TBC}}(t)$ given by $(5.6),(5.7)$.

We shall verify that this kernel $f_{0}(t)$ satisfies the stability condition (5.3). To this end we rewrite it as

$$
f_{0}(t)=f_{0}^{\mathrm{TBC}}(t)-f_{0}^{\mathrm{TBC}}(t) H(t-T) .
$$

Hence, its Laplace transform reads

$$
\begin{aligned}
\hat{f}_{0}(s) & =\hat{f}_{0}^{\mathrm{TBC}}(s)-\int_{T}^{\infty} f_{0}^{\mathrm{TBC}}(t) e^{-s t} d t \\
& =\sqrt{2} e^{-\frac{\pi}{4} i+\sqrt[+]{s}}+\frac{1}{\sqrt{2 \pi}} e^{-\frac{\pi}{4} i} \int_{T}^{\infty} t^{-\frac{3}{2}} e^{-s t} d t \\
& =\frac{1}{\sqrt{T}}\left(\sqrt{2} e^{-\frac{\pi}{4} i \sqrt[+]{\tilde{s}}}+\frac{1}{\sqrt{2 \pi}} e^{-\frac{\pi}{4} i} \int_{1}^{\infty} \tau^{-\frac{3}{2}} e^{-\tilde{s} \tau} d \tau\right), \quad s=\alpha_{1}+i \xi, \xi \in \mathbb{R},
\end{aligned}
$$

where $\tilde{s}=\tilde{\alpha}_{1}+i \tilde{\xi}, \tilde{\alpha}_{1}=\alpha_{1} T, \tilde{\xi}=\xi T, \tau=t / T$. It is easy to find numerically that (5.9) with $T=1$ satisfies the stability condition (5.3) if $\tilde{\alpha}_{1} \geq 0.23$. For general $T>0$ condition (5.3) therefore holds for $\alpha_{1}(T)=\tilde{\alpha}_{1} / T$. Note that $\alpha_{1}(T) \rightarrow 0$ for $T \rightarrow \infty$, which corresponds to Example 5.1.

Discretization. In the discrete case we shall use the following standard notation for finite difference operators:

$$
\Delta^{+} \psi_{j}=\psi_{j+1}-\psi_{j}, \quad \Delta^{-} \psi_{j}=\psi_{j}-\psi_{j-1}, \quad \Delta^{2} \psi_{j}=\psi_{j+1}-2 \psi_{j}+\psi_{j-1} .
$$

We are concerned with the stability (as $\Delta x, \Delta t \rightarrow 0$ ) of the Crank-Nicolson scheme for the Schrödinger equation (1.8) along with convolution-type BCs:

$$
\left\{\begin{aligned}
-i R\left(\psi_{j, n+1}-\psi_{j, n}\right) & =\Delta^{2}\left(\psi_{j, n+1}+\psi_{j, n}\right)-w V_{j, n+\frac{1}{2}}\left(\psi_{j, n+1}+\psi_{j, n}\right), \\
j=1, \ldots, J-1, & \\
\psi_{j, 0} & =\psi^{I}\left(x_{j}\right), j=0,1,2, \ldots, J-1, J ; \\
\text { with } \psi_{0,0} & =\psi_{1,0}=\psi_{J-1,0}=\psi_{J, 0}=0, \\
\Delta^{+} \hat{\psi}_{0}(z) & =\left(\hat{g}_{0}(z)-1\right) \hat{\psi}_{0}(z), \quad\left|\hat{g}_{0}(z)\right|>1, \\
\Delta^{-} \hat{\psi}_{J}(z) & =-\left(\hat{g}_{J}(z)-1\right) \hat{\psi}_{J}(z), \quad\left|\hat{g}_{J}(z)\right|>1,
\end{aligned}\right.
$$

where $\hat{g}_{0}(z), \hat{g}_{J}(z)$ are given functions. Alternatively, the two BCs can be written as

$$
\begin{array}{r}
\Delta^{+} \psi_{0, n}=\psi_{0, n} * \tilde{g}_{0, n}=\sum_{k=0}^{n} \psi_{0, k} \tilde{g}_{0, n-k} ; \quad \tilde{g}_{0, n}:=g_{0, n}-\delta_{0, n}, \\
\Delta^{-} \psi_{J, n}=-\psi_{J, n} * \tilde{g}_{J, n}=-\sum_{k=0}^{n} \psi_{J, k} \tilde{g}_{J, n-k} ; \quad \tilde{g}_{J, n}:=g_{J, n}-\delta_{0, n} .
\end{array}
$$


We assume that the $Z$-transforms of the given sequences $\left\{g_{j, n}\right\} ; j=0, J, \hat{g}_{j}(z):=$ $\mathcal{Z}\left\{g_{j, n}\right\}$ are analytic in a neighbourhood of $z=\infty$.

Typically, the BCs (5.11) should be a discrete approximation of the two continuous, convolution-BCs in (5.1). Hence the two functions $\hat{g}_{j} ; j=0, J$ are of course functions of $z, \Delta x$, and $\Delta t$, just as in the DTBCs (1.12a), (1.12b). In this example their functional dependence is of the form

$$
\hat{\ell}_{j} \equiv \hat{g}_{j}(z, \Delta x, \Delta t)=\hat{h}_{j}(y) \quad \text { with } y=y(z, \Delta x, \Delta t)=\frac{R}{2}\left(\frac{z-1}{z+1}+i \nu_{j}\right),
$$

where $\nu_{j}=\Delta t V_{j} / 2$ depends on the external potentials $V_{0}$ and $V_{J}$. In analogy to (1.9) we assume $V_{j}=V_{-}$for $j \leq 0$ and $V_{j}=V_{+}$for $j \geq J$.

Note that $y$ appearing in these discrete BCs is just the symbol of $\partial_{t}+i V$ in the Crank-Nicolson scheme, and the $Z$-transformed equation (1.8) can be recast in the exterior domain (i.e. for $j \leq 0$ or $j \geq J$ ) as

$$
i y \hat{\psi}_{j}(z)=-\frac{\Delta^{2}}{2} \hat{\psi}_{j}(z)
$$

Next we shall specify the typical $\Delta x$ - and $\Delta t$-dependence of general transformed boundary kernels $\hat{g}_{j}$. For arbitrary functions $f_{0}, f_{X}$ the BCs in (5.1) are usually not perfectly transparent. Therefore, their natural discretization cannot be obtained by calculating the discrete Dirichlet-to-Neumann map for the fully discretized whole space problem, as it was done in $[5,20]$ to derive the DTBCs.

After replacing $\psi_{x}$ in the BCs (5.1) by a finite difference quotient we now discuss the time discretization of their convolution integrals: A very natural method is based on the operational quadrature of $[26,27]$ by using the same time discretization for the $\mathrm{BCs}$ as for the evolution equation, i.e. the trapezoidal rule in our case. We shall hence assume that the transformed boundary kernels in (5.10) are also of the form

$$
\hat{g}_{j}(z, \Delta x, \Delta t)=\hat{h}_{j}(y),
$$

with $y$ given in (5.12) and some appropriate function $\hat{h}_{j}$. We remark that this approach also reproduces the DTBCs (1.10)-(1.12b). In the Transformation rule 3.1 we had already assumed this form (5.13) when calculating the explicit $\Delta x$ - and $\Delta t$ dependence of the sum-of-exponentials-BCs derived in $\S 3$.

$\ell^{2}-$ a-priori estimate of discrete solution. In the following lemma we shall derive an a-priori estimate for the temporal growth of the solution to (5.10) with $\Delta x$ and $\Delta t$ considered fixed. This discrete analogue of Proposition 5.1 will then be the key ingredient for our stability result (given in Theorem 5.1 and Theorem 5.2 below). We shall use the discrete $L^{2}$-norm:

$$
\left\|\psi_{n}\right\|_{2}^{2}:=\Delta x \sum_{j=1}^{J-1}\left|\psi_{j, n}\right|^{2} .
$$

Lemma 5.1. (Growth condition) Let the transformed boundary kernels $\hat{g}_{0}, \hat{g}_{J}$ satisfy

$$
\operatorname{Im} \hat{g}_{0}\left(\beta e^{i \varphi}\right) \leq 0, \quad \operatorname{Im} \hat{g}_{J}\left(\beta e^{i \varphi}\right) \leq 0, \quad \forall 0 \leq \varphi \leq 2 \pi,
$$


for some (sufficiently large) $\beta \geq 1$. Assume also that $\hat{g}_{0}, \hat{g}_{J}$ are analytic for $|z| \geq \beta$. Then, the solution of (5.10) satisfies the a-priori estimate

$$
\left\|\psi_{n}\right\|_{2} \leq\left\|\psi_{0}\right\|_{2} \beta^{n}, \quad n \in \mathbb{N} .
$$

Proof. The proof is analogous to that of Proposition 5.1 and it is based on a discrete energy estimate for the new variable

$$
\phi_{j, n}:=\psi_{j, n} \beta^{-n},
$$

which satisfies the equation

$$
\begin{aligned}
-i R\left(\phi_{j, n+1}-\phi_{j, n}\right)= & \left(\Delta^{2}-w V_{j, n+\frac{1}{2}}\right)\left(\phi_{j, n+1}+\phi_{j, n}\right) \\
& +(\beta-1)\left(\Delta^{2}-w V_{j, n+\frac{1}{2}}+i R\right) \phi_{j, n+1} ; \quad j=1, \ldots, J-1, \\
\phi_{j, 0}= & \psi_{j, 0} ; \quad j=0, \ldots, J \\
\Delta^{+} \hat{\phi}_{0}(z)= & \left(\hat{g}_{0}(\beta z)-1\right) \hat{\phi}_{0}(z), \\
\Delta^{-} \hat{\phi}_{J}(z)= & -\left(\hat{g}_{J}(\beta z)-1\right) \hat{\phi}_{J}(z) .
\end{aligned}
$$

In physical space, the two BCs can be written as

$$
\begin{aligned}
& \Delta^{+} \phi_{0, n}=\phi_{0, n} * \frac{\tilde{g}_{0, n}}{\beta^{n}}=\sum_{k=0}^{n} \phi_{0, k}\left(\tilde{g}_{0, n-k} \beta^{k-n}\right), \\
& \Delta^{-} \phi_{J, n}=-\phi_{J, n} * \frac{\tilde{g}_{J, n}}{\beta^{n}}=-\sum_{k=0}^{n} \phi_{J, k}\left(\tilde{g}_{J, n-k} \beta^{k-n}\right) .
\end{aligned}
$$

First we multiply (5.17a) by $\bar{\phi}_{j, n} / \beta$ and its complex conjugate by $\phi_{j, n+1}$ :

$$
\begin{aligned}
-i R \bar{\phi}_{j, n}\left(\phi_{j, n+1}-\phi_{j, n}\right)= & \bar{\phi}_{j, n}\left(\Delta^{2}-w V_{j, n+\frac{1}{2}}\right)\left(\phi_{j, n+1}+\phi_{j, n}\right) \\
& +\left(\beta^{-1}-1\right) \bar{\phi}_{j, n}\left(\Delta^{2}-w V_{j, n+\frac{1}{2}}-i R\right) \phi_{j, n}, \\
i R \phi_{j, n+1}\left(\bar{\phi}_{j, n+1}-\bar{\phi}_{j, n}\right)= & \phi_{j, n+1}\left(\Delta^{2}-w V_{j, n+\frac{1}{2}}\right)\left(\bar{\phi}_{j, n+1}+\bar{\phi}_{j, n}\right) \\
& +(\beta-1) \phi_{j, n+1}\left(\Delta^{2}-w V_{j, n+\frac{1}{2}}-i R\right) \bar{\phi}_{j, n+1} .
\end{aligned}
$$

Note that we used equation (5.17a) to modify the last term of (5.19). Next we subtract (5.20) from (5.19), sum from $j=1$ to $j=J-1$, and take imaginary parts. After a simple, but lengthy calculation we obtain:

$$
\begin{gathered}
\sum_{j=1}^{J-1}\left(\left|\phi_{j, n+1}\right|^{2}-\left|\phi_{j, n}\right|^{2}\right)=-\left(1-\beta^{-1}\right) \sum_{j=1}^{J-1}\left|\phi_{j, n}\right|^{2}-(\beta-1) \sum_{j=1}^{J-1}\left|\phi_{j, n+1}\right|^{2} \\
+\frac{1}{\beta R} \operatorname{Im}\left[\left(\bar{\phi}_{0, n}+\beta \bar{\phi}_{0, n+1}\right) \Delta^{+}\left(\phi_{0, n}+\beta \phi_{0, n+1}\right)\right. \\
\left.-\left(\bar{\phi}_{J, n}+\beta \bar{\phi}_{J, n+1}\right) \Delta^{-}\left(\phi_{J, n}+\beta \phi_{J, n+1}\right)\right]
\end{gathered}
$$


Summing (5.21) from $n=0$ to $n=N$ yields (note that $\beta \geq 1$ ):

$$
\begin{aligned}
\left\|\phi_{N+1}\right\|_{2}^{2} \leq\left\|\phi_{0}\right\|_{2}^{2}+\frac{\Delta x}{\beta^{2} R} \operatorname{Im} \sum_{n=0}^{N}\left[\left(\bar{\phi}_{0, n}+\beta \bar{\phi}_{0, n+1}\right) \Delta^{+}\left(\phi_{0, n}+\beta \phi_{0, n+1}\right)\right. \\
\left.-\left(\bar{\phi}_{J, n}+\beta \bar{\phi}_{J, n+1}\right) \Delta^{-}\left(\phi_{J, n}+\beta \phi_{J, n+1}\right)\right] \\
=\left\|\phi_{0}\right\|_{2}^{2}+\frac{\Delta x}{\beta^{2} R} \operatorname{Im} \sum_{n=0}^{N}\left[\left(\bar{\phi}_{0, n}+\beta \bar{\phi}_{0, n+1}\right)\left(\phi_{0, n}+\beta \phi_{0, n+1}\right) * \frac{\tilde{g}_{0, n}}{\beta^{n}}\right. \\
\left.+\left(\bar{\phi}_{J, n}+\beta \bar{\phi}_{J, n+1}\right)\left(\phi_{J, n}+\beta \phi_{J, n+1}\right) * \frac{\tilde{g}_{J, n}}{\beta^{n}}\right] .
\end{aligned}
$$

For the last identity we used the BCs (5.18) and $\phi_{0,0}=\phi_{J, 0}=0$.

To finish the proof it remains to determine the sign of the last term in (5.22). To this end we define (for $N$ fixed) the two sequences,

$$
\begin{aligned}
& u_{n}:= \begin{cases}\phi_{0, n}+\beta \phi_{0, n+1}, & n=0, \ldots, N, \\
0, & n>N,\end{cases} \\
& v_{n}:=u_{n} * \frac{\tilde{g}_{0, n}}{\beta^{n}}=\sum_{k=0}^{n} u_{k} \frac{\tilde{g}_{0, n-k}}{\beta^{n-k}}, \quad n \in \mathbb{N}_{0} .
\end{aligned}
$$

The $Z$-transform $\mathcal{Z}\left\{u_{n}\right\}=\hat{u}(z)$ is analytic for $|z|>0$, since it is a finite sum. The $Z$-transform $\mathcal{Z}\left\{v_{n}\right\}$ then satisfies $\hat{v}(z)=\left(\hat{g}_{0}(\beta z)-1\right) \hat{u}(z)$ and is analytic for $|z| \geq 1$. Using Plancherel's Theorem for $Z$-transforms (cf. $\S 38$ in [18]) we have

$$
\begin{aligned}
\sum_{n=0}^{N} v_{n} \bar{u}_{n}=\sum_{n=0}^{\infty} v_{n} \bar{u}_{n} & =\frac{1}{2 \pi} \int_{0}^{2 \pi} \hat{v}\left(e^{i \varphi}\right) \overline{\hat{u}\left(e^{i \varphi}\right)} d \varphi \\
& =\frac{1}{2 \pi} \int_{0}^{2 \pi}\left|\hat{u}\left(e^{i \varphi}\right)\right|^{2}\left(\hat{g}_{0}\left(\beta e^{i \varphi}\right)-1\right) d \varphi
\end{aligned}
$$

Using (5.23) for the two boundary terms in (5.22) now gives:

$$
\begin{aligned}
\left\|\phi_{N+1}\right\|_{2}^{2} \leq\left\|\phi_{0}\right\|_{2}^{2}+ & \frac{\Delta x}{2 \pi \beta^{2} R} \int_{0}^{2 \pi}\left[\left|\left(1+\beta e^{i \varphi}\right) \hat{\phi}_{0}\left(e^{i \varphi}\right)\right|^{2} \operatorname{Im}\left(\hat{g}_{0}\left(\beta e^{i \varphi}\right)-1\right)\right. \\
& \left.+\left|\left(1+\beta e^{i \varphi}\right) \hat{\phi}_{J}\left(e^{i \varphi}\right)\right|^{2} \operatorname{Im}\left(\hat{g}_{J}\left(\beta e^{i \varphi}\right)-1\right)\right] d \varphi
\end{aligned}
$$

Our assumptions on $\hat{g}_{0}$ and $\hat{g}_{J}$ hence imply

$$
\left\|\phi_{N}\right\|_{2} \leq\left\|\phi_{0}\right\|_{2}, \quad \forall N \geq 0,
$$

and the result follows.

REMARK 5. Above we have assumed that the two transformed boundary kernels $\hat{g}_{j} ; j=$ $0, J$ - as Z-transforms - are analytic for $|z| \geq \beta$. Hence their imaginary parts are harmonic functions there. Since the average of $\hat{g}_{j}(z)$ on the circles $z=\beta e^{i \varphi}$ equals $g_{j, 0}=\hat{g}_{j}(z=\infty)$, condition (5.15) implies $\operatorname{Im} \hat{g}_{j}(z=\infty) \leq 0 ; j=0, J$. Then we have the following simple consequence of the maximum principle for the Laplace equation:

If condition (5.15) holds for some $\beta_{0}$, it also holds for all $\beta>\beta_{0}$. 
Example 5.3. (Discrete counterpart of 5.1) For the exact DTBC (1.17) (with $\hat{g}_{j}=\hat{\ell}_{j}, j=0, J$ ) the stability condition (5.15) is clearly satisfied for $\beta=1$ (see Figure 5.1). In fact the DTBC satisfies (5.15) for all $\Delta x$ and $\Delta t$. Hence, the discrete $L^{2}$-norm of $\psi_{n}$ always decreases monotonically in time. And in this case the $L^{2}$-stability of the scheme (5.10) is trivial.

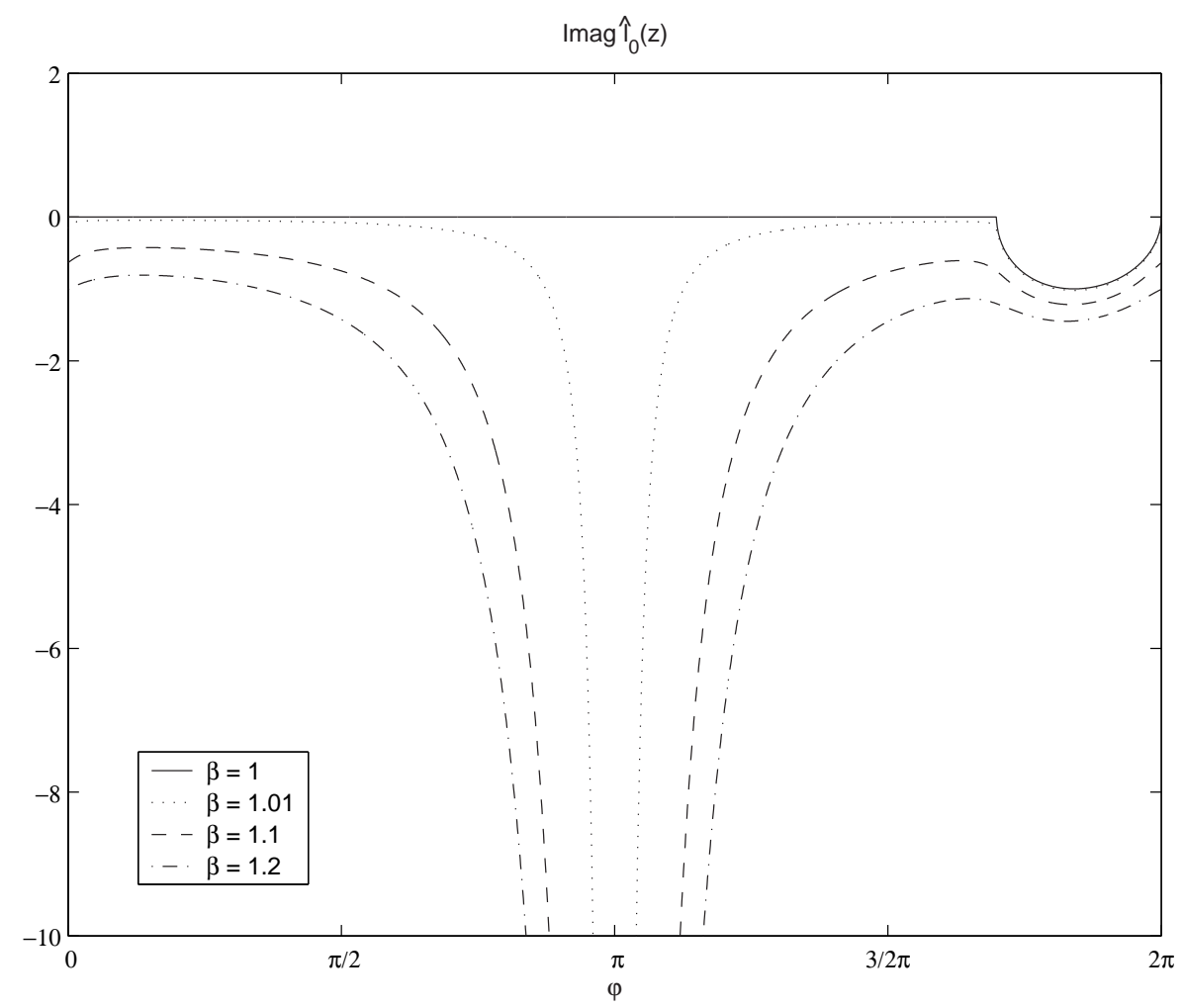

FIG. 5.1. The imaginary part of $\hat{\ell}_{0}(z)$ is non-positive on the unit circle $z=e^{i \varphi}, 0 \leq \varphi \leq 2 \pi$ (and hence also for $\beta \geq 1$ ). $\Delta x=1 / 160, \Delta t=2 \cdot 10^{-5}, V \equiv 0$.

Example 5.4. (Discrete counterpart of Example 5.2) As a second example we consider a simplification of the exact DTBC (1.17), where the convolution coefficients $s_{j, n}$ are cut off for $n \geq N$ (cf. [20] for a discussion of the accuracy and practical relevance of these $\mathrm{BCs}$ ). The corresponding $Z$-transform,

$$
\hat{\ell}_{j}^{(N)}(z)=\frac{z}{z+1} \hat{s}_{j}^{(N)}(z)=\frac{z}{z+1} \sum_{n=0}^{N} s_{j, n} z^{-n}
$$

satisfies the growth condition (5.15) only for $\beta \geq 1.25$ if $N=10$ (see Figure 5.2). (The continuous analogue of such a cut-off kernel was given in (5.8)).

$\ell^{2}$-stability. Now we turn to the stability of the numerical scheme (5.10) as $\Delta t \rightarrow 0$. To this end we shall derive uniform estimates for $\left\|\psi_{n}\right\|_{2}$ (as $\Delta t \rightarrow 0$ and $0 \leq n \Delta t \leq T)$ by using Lemma 5.1. This lemma bounds the exponential growth of solutions to the numerical scheme for a fixed discretization $(\Delta x, \Delta t)$ and it will be 


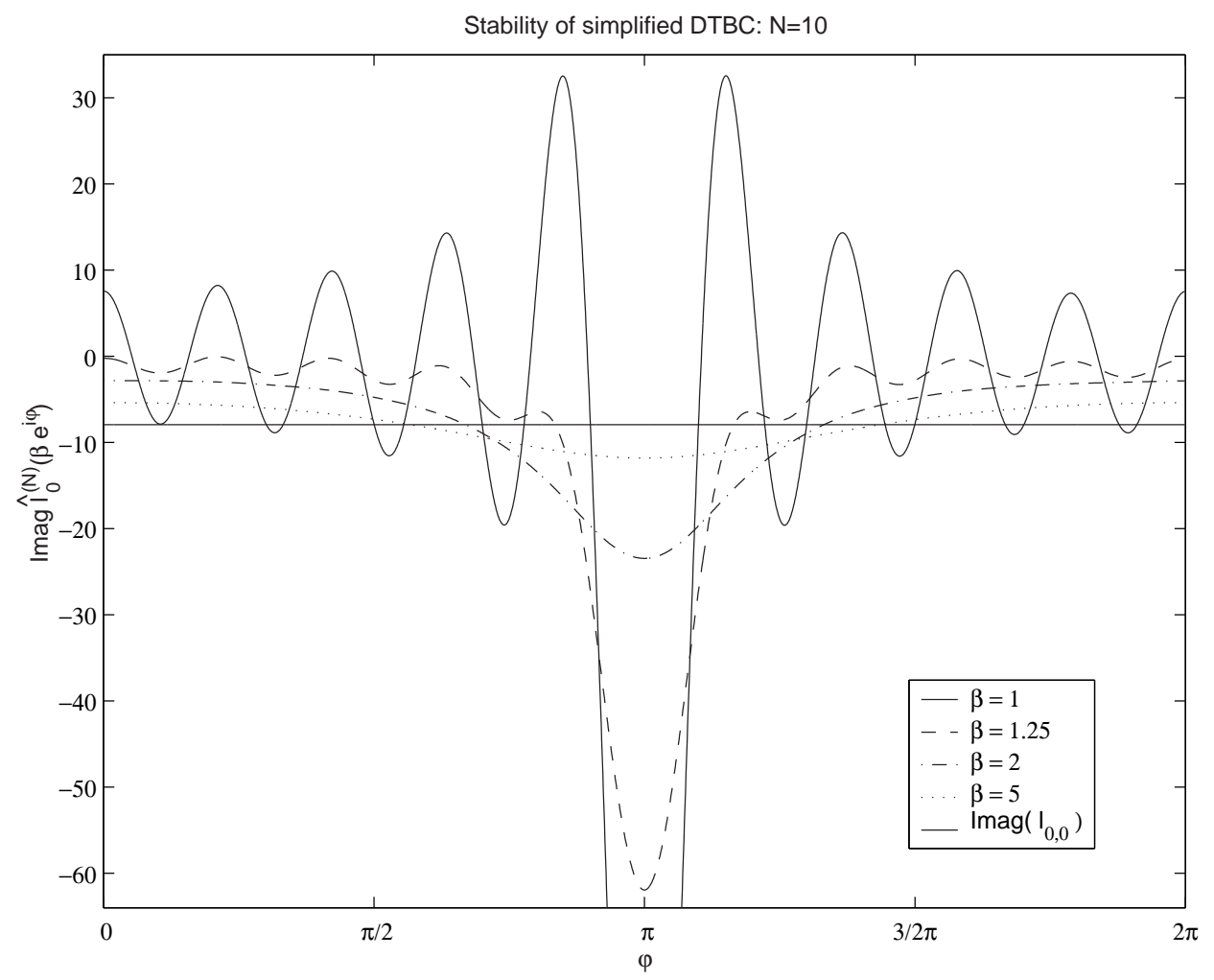

FIG. 5.2. Growth condition $\operatorname{Im} \hat{\ell}_{0}^{(N)}\left(z=\beta e^{i \varphi}\right) \leq 0$ for simplified discrete transparent boundary conditions with cut-off after $N=10$ convolution coefficients, $\Delta x=1 / 160, \Delta t=2 \cdot 10^{-5}, V \equiv 0$. The stability condition (5.15) is satisfied for $\beta \geq 1.25$, and Im $\ell_{0,0}<0$ holds.

applied for each value of $\Delta t$ along with a corresponding radius $\beta(\Delta t)$. Since the case $\beta(\Delta t) \equiv 1$ is trivial (cf. the DTBC illustrated in Figure 5.1) we focus on the case when $\beta=\beta(\Delta t)>1$. For estimating $\left\|\psi_{n}\right\|_{2}$ we shall require that there exists a fixed $\rho \geq 0$ such that

$$
\beta(\Delta t) \leq e^{\rho \Delta t}, \quad \forall 0<\Delta t \leq \Delta t_{0}
$$

Using (5.16) this would then yield our final stability estimate

$$
\left\|\psi_{n}\right\|_{2} \leq\left\|\psi_{0}\right\|_{2} \beta(\Delta t)^{n} \leq\left\|\psi_{0}\right\|_{2} e^{\rho n \Delta t} \leq\left\|\psi_{0}\right\|_{2} e^{\rho T}, \quad \forall n \Delta t \leq T .
$$

Next we shall discuss conditions on the boundary function $\hat{g}_{j}(z)$ for one fixed discretization $\left(\Delta x_{0}, \Delta t_{0}\right)$, which imply that the stability estimate (5.15) holds for all $0<\Delta t \leq \Delta t_{0}$ on circles with radius $\beta(\Delta t)=e^{\rho \Delta t}$, which decrease as $\Delta t \rightarrow 0$. In the following three cases we shall assume different behaviours of the function $\Delta x=\Delta x(\Delta t)($ as $\Delta t \rightarrow 0)$ that permit to prove stability of the scheme (5.10):

Case 1. $R=4 \Delta x^{2} / \Delta t=$ const as $\Delta t \rightarrow 0$, and $V_{j}=0$ :

Because of the functional dependence (5.12), $\hat{g}_{j}$ is here independent of $\Delta t$, and therefore $\beta$ in (5.15) would also be independent of $\Delta t$. Hence, the stability estimate (5.26) 
can only be obtained if the condition (5.15) holds for $\beta=\beta\left(\Delta t_{0}\right)=1$.

Case 2. $\Delta x=\Delta x_{0}=$ const and $V_{j} \in \mathbb{R}$ :

THEOREM 5.1. Let the transformed boundary kernels $\hat{g}_{0}, \hat{g}_{J}$ satisfy

$$
\left.\operatorname{Im} \hat{g}_{0}\left(z, \Delta x_{0}, \Delta t_{0}\right)\right|_{\mathcal{C}_{1}} \leq 0,\left.\quad \operatorname{Im} \hat{g}_{J}\left(z, \Delta x_{0}, \Delta t_{0}\right)\right|_{\mathcal{C}_{1}} \leq 0
$$

for some fixed $\Delta x_{0}$ and $\Delta t_{0}$. Here, the circle $\mathcal{C}_{1}$ is defined by

$$
z=\frac{\beta_{0}-1}{2}+\frac{\beta_{0}+1}{2} e^{i \varphi}, \quad 0 \leq \varphi \leq 2 \pi
$$

(cf. Figure 5.3) with some sufficiently large $\beta_{0}=e^{\rho \Delta t_{0}} \geq 1$ (note that $z=-1$ and $\left.z=\beta_{0} \in \mathcal{C}_{1}\right)$.

Then, the stability estimate (5.26) holds for all $0<\Delta t \leq \Delta t_{0}$ and $\Delta x=\Delta x_{0}$.

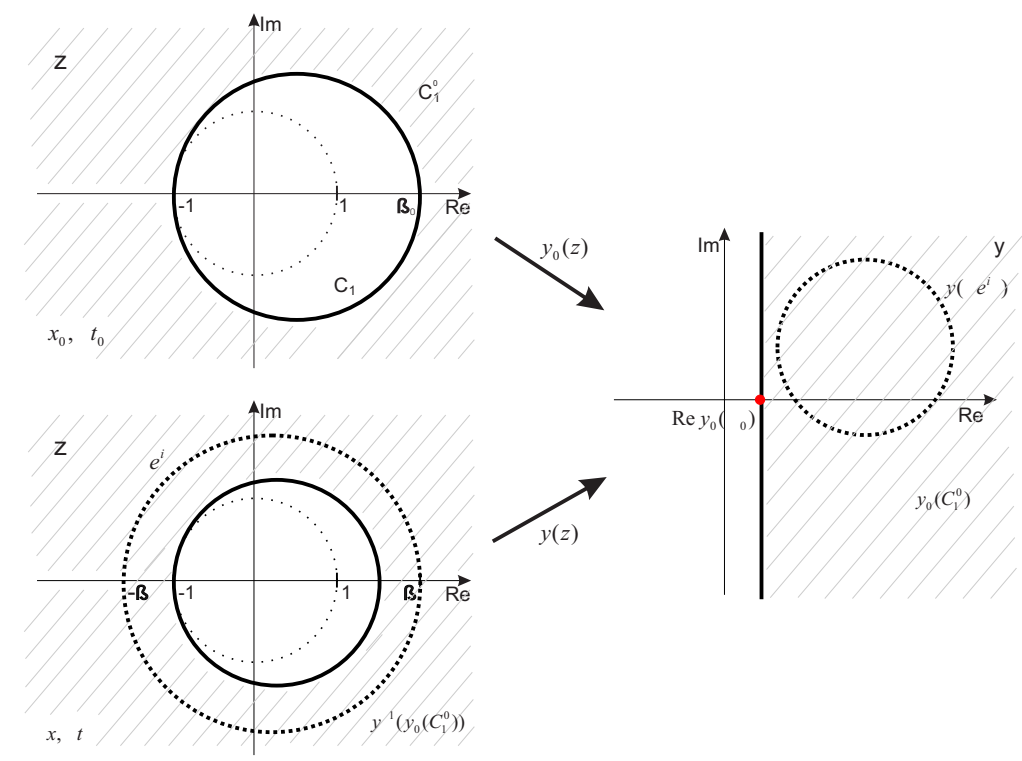

FIG. 5.3. Illustration of the 2 maps $y_{0}(z)$ and $y(z)$ from the proof of Theorem 5.1: The circle $z=\beta e^{i \varphi}$ (dashed line) is mapped onto $y\left(\beta e^{i \varphi}\right)$ which lies inside the (shaded) stability region $y_{0}\left(\mathcal{C}_{1}^{o}\right)$.

Proof. Following the above remark (after the proof of Lemma 5.1) we first note that condition (5.27) implies

$$
\operatorname{Im} \hat{g}_{j}\left(z, \Delta x_{0}, \Delta t_{0}\right) \leq 0 \quad \forall z \in \mathcal{C}_{1}^{o}
$$

where $\mathcal{C}_{1}^{o}$ denotes the part of the complex plane "outside" of the circle $\mathcal{C}_{1}$. The idea of the proof is to conclude that (5.28) implies

$$
\operatorname{Im} \hat{g}_{j}\left(z=\beta(\Delta t) e^{i \varphi}, \Delta x_{0}, \Delta t\right) \leq 0, \quad \forall \quad 0<\Delta t \leq \Delta t_{0}, \quad 0 \leq \varphi \leq 2 \pi,
$$

with $\beta(\Delta t)=e^{\rho \Delta t}$. To this end we shall compare the images of $\mathcal{C}_{1}^{o}$ and the circles $\beta(\Delta t) e^{i \varphi}$ from (5.29) under the respective maps $y_{0}(z)=y\left(z, \Delta x_{0}, \Delta t_{0}\right)$ and $y(z)=$ $y\left(z, \Delta x_{0}, \Delta t\right)$ from (5.12). 
We first consider the rational map

$$
y_{0}(z)=\frac{2 \Delta x_{0}^{2}}{\Delta t_{0}}\left(\frac{z-1}{z+1}+i \frac{\Delta t_{0} V_{j}}{2}\right): \mathbb{C} \rightarrow \mathbb{C}
$$

from (5.12). $y_{0}\left(\mathcal{C}_{1}^{o}\right)$, the image of $\mathcal{C}_{1}^{o}$ is the set $y \in \mathbb{C}$ with

$$
\operatorname{Re} y \geq \operatorname{Re} y_{0}\left(z=\beta_{0}\right)=\frac{2 \Delta x_{0}^{2}}{\Delta t_{0}} \frac{\beta_{0}-1}{\beta_{0}+1}=\frac{2 \Delta x_{0}^{2}}{\Delta t_{0}} \tanh \frac{\rho \Delta t_{0}}{2} \text {. }
$$

In order to verify the estimate (5.15) with $\beta(\Delta t)=e^{\rho \Delta t}$ for $0<\Delta t \leq \Delta t_{0}$ we calculate:

$$
\hat{g}_{j}\left(z=e^{\rho \Delta t+i \varphi}, \Delta x_{0}, \Delta t\right)=\hat{h}_{j}(y(\Delta t, \varphi)),
$$

with

$$
y(\Delta t, \varphi):=\frac{2 \Delta x_{0}^{2}}{\Delta t}\left[\frac{e^{\rho \Delta t+i \varphi}-1}{e^{\rho \Delta t+i \varphi}+1}+i \frac{\Delta t V_{j}}{2}\right] .
$$

Using the rational map $y(z)=\frac{2 \Delta x_{0}^{2}}{\Delta t}\left(\frac{z-1}{z+1}+i \frac{\Delta t V_{j}}{2}\right)$ we see that

$$
\operatorname{Re} y(\Delta t, \varphi) \geq \operatorname{Re} y(\Delta t, 0)=\frac{2 \Delta x_{0}^{2}}{\Delta t} \tanh \frac{\rho \Delta t}{2}, \quad \forall 0 \leq \varphi \leq 2 \pi .
$$

Due to the monotonicity of the function $\tanh x / x$ we finally obtain for the argument of $\hat{h}_{j}$ in (5.32):

$$
\begin{aligned}
\operatorname{Re} y(\Delta t, \varphi) & \geq \frac{2 \Delta x_{0}^{2}}{\Delta t_{0}} \tanh \frac{\rho \Delta t_{0}}{2} \\
& =\operatorname{Re} y_{0}\left(z=e^{\rho \Delta t_{0}}=\beta_{0}\right), \quad \forall 0<\Delta t \leq \Delta t_{0}, 0 \leq \varphi \leq 2 \pi .
\end{aligned}
$$

Hence,

$$
y_{0}^{-1}(y(\Delta t, \varphi)) \in \mathcal{C}_{1}^{o}, \quad \forall 0<\Delta t \leq \Delta t_{0}, 0 \leq \varphi \leq 2 \pi
$$

(5.28) now shows that

$$
\operatorname{Im} \hat{g}_{j}\left(\beta e^{i \varphi}, \Delta x_{0}, \Delta t\right) \leq 0
$$

holds with $\beta=e^{\rho \Delta t}$, and (5.26) follows.

Summarizing, the idea of the proof is based on the fact that all circles $\beta(\Delta t) e^{i \varphi}$ (with $0<\Delta t \leq \Delta t_{0}$ ) are mapped via $y_{0}^{-1} \circ y$ into $\mathcal{C}_{1}^{o}$ (cf. Figure 5.3).

Case 3. $\Delta x=\alpha \Delta t^{\gamma}$ (with $\alpha=\Delta x_{0} / \Delta t_{0}^{\gamma}, \gamma \geq 1$ ) and $V_{j} \in \mathbb{R}$ :

THEOREM 5.2. (a) Let $V_{j}=0$ and let the transformed boundary kernels $\hat{g}_{0}, \hat{g}_{J}$ satisfy

$$
\left.\operatorname{Im} \hat{g}_{0}\left(z, \Delta x_{0}, \Delta t_{0}\right)\right|_{\mathcal{C}_{2}} \leq 0,\left.\quad \operatorname{Im} \hat{g}_{J}\left(z, \Delta x_{0}, \Delta t_{0}\right)\right|_{\mathcal{C}_{2}} \leq 0
$$

for some $\Delta x_{0}$ and $\Delta t_{0}$. Here, the circle $\mathcal{C}_{2}$ is defined by

$$
z=-\frac{\beta_{0}-1}{2}+\frac{\beta_{0}+1}{2} e^{i \varphi}, \quad 0 \leq \varphi \leq 2 \pi
$$


and some sufficiently large $\beta_{0}=e^{\rho \Delta t_{0}} \geq 1$ (note that $z=-\beta_{0}$ and $z=1 \in \mathcal{C}_{2}$ ).

Then, the stability estimate (5.26) holds for all $0<\Delta t \leq \Delta t_{0}$ and $\Delta x=\alpha \Delta t^{\gamma}$.

(b) Let $V_{j} \in \mathbb{R}$ and let $\hat{g}_{0}^{0}, \hat{g}_{J}^{0}$, the transformed boundary kernels pertaining to $V_{j}=0$, satisfy condition (5.33) for some $\Delta x_{0}, \Delta t_{0}$, and $\beta_{0}=e^{\rho \Delta t_{0}} \geq 1$.

Then, the stability estimate (5.26) holds for all $0<\Delta t \leq \Delta t_{1}$ (with some $0<$ $\left.\Delta t_{1} \leq \Delta t_{0}\right)$ and $\Delta x=\alpha \Delta t^{\gamma}$.

Proof. Part (a):

We follow the strategy of the previous proof and first note that condition (5.33) implies

$$
\operatorname{Im} \hat{g}_{j}\left(z, \Delta x_{0}, \Delta t_{0}\right) \leq 0 \quad \forall z \in \mathcal{C}_{2}^{o},
$$

where $\mathcal{C}_{2}^{o}$ denotes the "outside" of circle $\mathcal{C}_{2} . y_{0}\left(\mathcal{C}_{2}^{o}\right)$, its image under the rational map

$$
y_{0}(z)=\frac{2 \Delta x_{0}^{2}}{\Delta t_{0}} \frac{z-1}{z+1}
$$

is characterized by $y \in \mathbb{C}$ with

$$
\left|y+\frac{\Delta x_{0}^{2}}{\Delta t_{0}} \frac{\beta_{0}+1}{\beta_{0}-1}\right| \leq \frac{\Delta x_{0}^{2}}{\Delta t_{0}} \frac{\beta_{0}+1}{\beta_{0}-1} .
$$

The disk $y_{0}\left(\mathcal{C}_{2}^{o}\right)$ is symmetric to the real axis, with left vertex at $y_{l}=0$ and right vertex at $y_{r}=\frac{2 \Delta x_{0}^{2}}{\Delta t_{0}} \operatorname{coth} \frac{\rho \Delta t_{0}}{2}$.

In order to verify the estimate (5.15) with $\beta(\Delta t)=e^{\rho \Delta t}$ for $0<\Delta t \leq \Delta t_{0}$ we calculate:

$$
\hat{g}_{j}\left(z=e^{\rho \Delta t+i \varphi}, \alpha \Delta t^{\gamma}, \Delta t\right)=\hat{h}_{j}(y(\Delta t, \varphi))
$$

with

$$
y(\Delta t, \varphi):=2 \alpha^{2} \Delta t^{2 \gamma-1} \frac{e^{\rho \Delta t+i \varphi}-1}{e^{\rho \Delta t+i \varphi}+1} .
$$

For fixed $0<\Delta t \leq \Delta t_{0}$ the set $\{y(\Delta t, \varphi) \mid 0 \leq \varphi \leq 2 \pi\}$ is a circle, lying symmetric to the real axis with left vertex at

$$
y(\Delta t, 0)=2 \alpha^{2} \Delta t^{2 \gamma-1} \tanh \frac{\rho \Delta t}{2}>y_{l}=0,
$$

and right vertex at

$$
y(\Delta t, \pi)=2 \alpha^{2} \Delta t^{2 \gamma-1} \operatorname{coth} \frac{\rho \Delta t}{2} \leq y_{r} .
$$

Therefore the circles $\{y(\Delta t, \varphi) \mid 0 \leq \varphi \leq 2 \pi\} \subset y_{0}\left(\mathcal{C}_{2}^{o}\right)$, for $0<\Delta t \leq \Delta t_{0}$.

Hence, (5.34) and (5.36) show that

$$
\operatorname{Im} \hat{g}_{j}\left(\beta e^{i \varphi}, \Delta x, \Delta t\right) \leq 0
$$

holds with $\beta=e^{\rho \Delta t}, \Delta x=\alpha \Delta t^{\gamma}$, and the stability estimate (5.26) follows.

Part (b): 


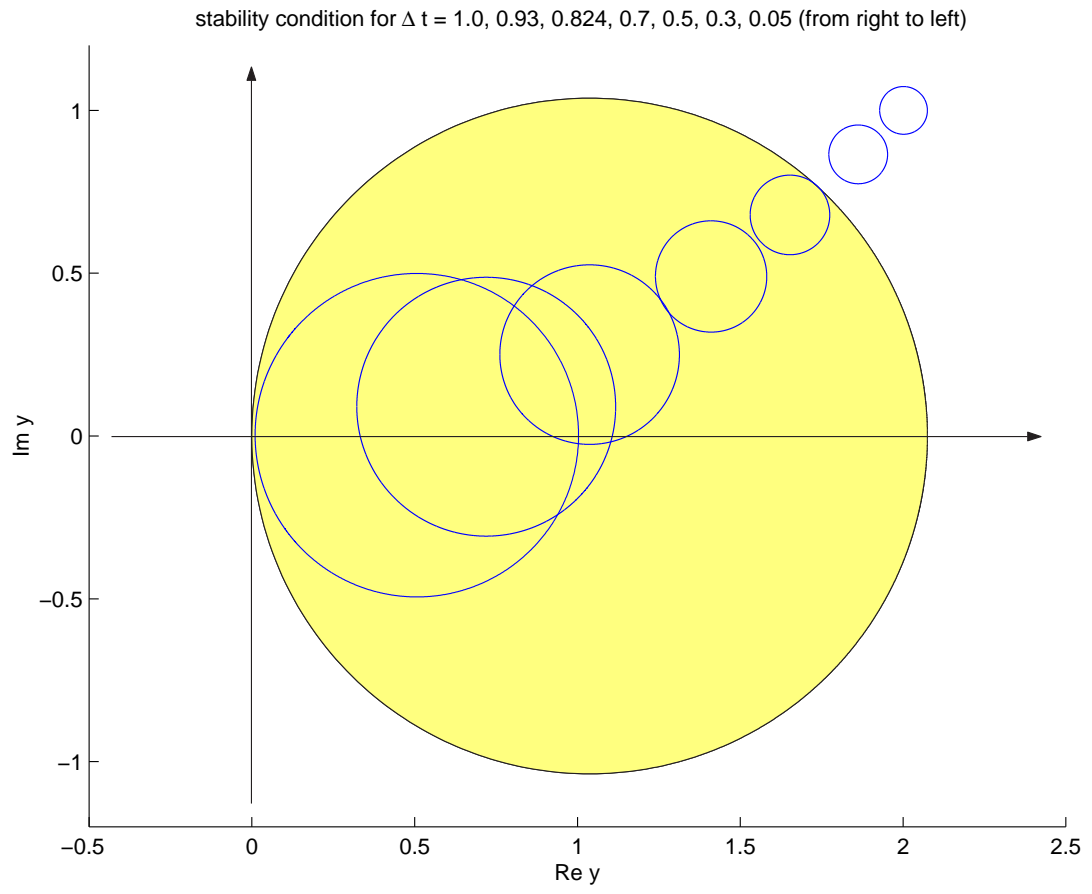

FIG. 5.4. Illustration of the proof of Theorem 5.2 (b): For $\Delta t \leq \Delta t_{1} \approx 0.82$ the (small) circles $\{y(\Delta t, \varphi) \mid 0 \leq \varphi \leq 2 \pi\}$ are inside the (shaded) stability region $y_{0}\left(\mathcal{C}_{2}^{o}\right)$. Parameters: $\Delta x_{0}=\Delta t_{0}=$ $V=\gamma=1, \rho=4$.

To verify the estimate (5.15) we have to show that $\operatorname{Im} \hat{g}_{j}\left(z=e^{\rho \Delta t+i \varphi}, \alpha \Delta t^{\gamma}, \Delta t\right)=$ $\operatorname{Im} \hat{h}_{j}(y(\Delta t, \varphi)) \leq 0$, with

$$
y(\Delta t, \varphi):=2 \alpha^{2} \Delta t^{2 \gamma-1}\left[\frac{e^{\rho \Delta t+i \varphi}-1}{e^{\rho \Delta t+i \varphi}+1}+i \frac{\Delta t V_{j}}{2}\right] .
$$

The circles $y(\Delta t, \varphi), 0 \leq \varphi \leq 2 \pi$ are only vertical shifts of the circles (5.38), and their left vertex is at

$$
y(\Delta t, 0)=\alpha^{2} \Delta t^{2 \gamma}\left(\rho+i V_{j}\right)+\mathcal{O}\left(\Delta t^{2 \gamma+1}\right) \quad \text { for } \Delta t \rightarrow 0 .
$$

Hence, the circles $\{y(\Delta t, \varphi) \mid 0 \leq \varphi \leq 2 \pi\} \subset y_{0}\left(\mathcal{C}_{2}^{o}\right)$, for $0<\Delta t \leq \Delta t_{1}$ (for some $0<\Delta t_{1} \leq \Delta t_{0}$; cf. Figure 5.4) and the stability estimate (5.26) follows.

We now illustrate the stability condition (5.27) of Case 2 and condition (5.33) of Case 3 for the exponential-sum-coefficients introduced in Section 3. The upper left graphic of Figure 5.3 shows the unit circle (dotted line) in the complex plane and the circle $\mathcal{C}_{1}$ (solid line), on which we shall check the stability condition.

Example 5.5. We consider the free 1D-Schrödinger equation with the discretization $\Delta x=1 / 160, \Delta t=2 \cdot 10^{-5}$. The transformed boundary kernel has the form $\hat{\tilde{\ell}}(z)=\frac{z}{z+1} \hat{\tilde{s}}(z)$, where the $Z$-transform of $\left\{\tilde{s}_{n}\right\}$ with $\nu=2$ reads

$$
\hat{\tilde{s}}(z)=s_{0}+s_{1} z^{-1}+\sum_{l=1}^{L} \frac{b_{l}}{q_{l} z-1} \frac{1}{q_{l} z}, \quad|z| \geq 1 .
$$


We recall that $\left|q_{l}\right|>1, l=1, \ldots, L$ (cf. Theorem 3.2(ii)), and hence all poles of $\hat{\tilde{s}}$ are inside the unit circle.

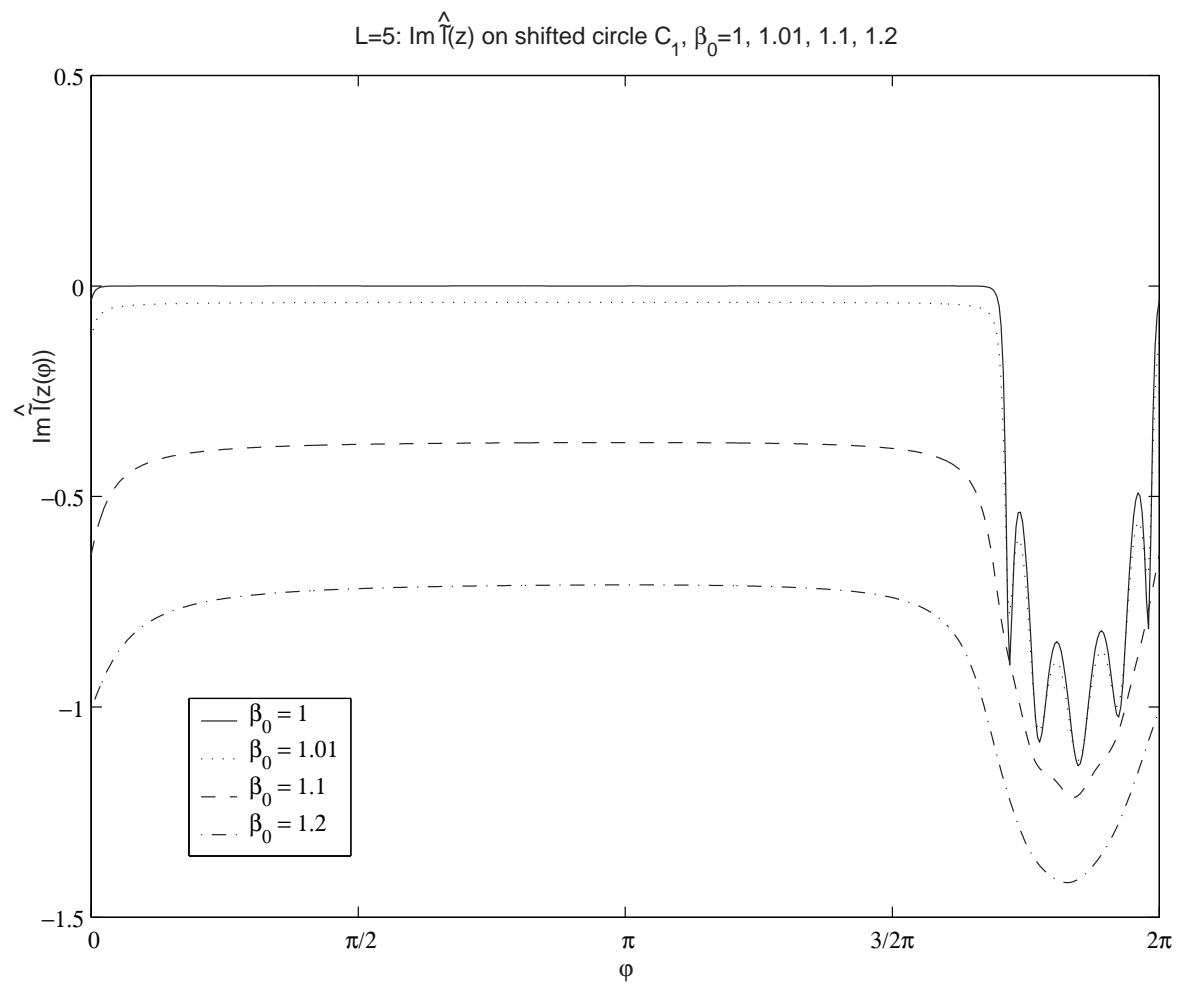

FIG. 5.5. Stability condition (5.27), i.e. Im $\hat{\tilde{\ell}}_{\mathcal{C}_{1}} \leq 0$ for exponential-sum-coefficients with $L=5$. The condition is not satisfied for $\beta_{0}=1$ but is satisfied for $\beta_{0} \geq 1.0000008$.

Figure 5.5 shows $\operatorname{Im} \hat{\tilde{\ell}}_{\mathcal{C}_{1}}$ for $L=5$ and various values of $\beta_{0}$. For $\beta_{0}=1, \operatorname{Im} \hat{\tilde{\ell}}$ is not always negative on the unit circle $\left(\max \operatorname{Im} \hat{\tilde{\ell}}_{\mathcal{C}_{1}}=3.06 x 10-6\right.$ if $\left.L=5\right)$. Thus the stability condition is not satisfied for $\beta_{0}=1$, but it is satisfied for lager values of $\beta_{0}$. Comparing these plots with the transformed boundary kernel of the exact DTBC (Figure 5.1 in Example 5.3) we observe that the 'valley' of the graph is at the same position. With increasing $\beta_{0}$ this valley becomes flatter and $\operatorname{Im} \hat{\tilde{\ell}}_{\mathcal{C}_{1}}$ converges to $\operatorname{Im}$ $s_{0} \approx-7.93$.

Note that the imaginary part of $\hat{\tilde{\ell}}=\hat{\tilde{s}} \frac{z}{z+1}$ has no singularity at $z=-1 \epsilon$ $\mathcal{C}_{1}$. This is due to the fact that $\operatorname{Re} \hat{s}(z=-1)=0$ (cf. (1.12a), (1.12b)) and its (reasonable) approximations (5.39) also satisfy $\operatorname{Re} \hat{\tilde{s}}(z=-1)=0$. Furthermore, $\operatorname{Re} \frac{z}{z+1}$ is continuous at $z=-1$ on the circle $\mathcal{C}_{1}$.

Similarly, Figure 5.6 shows that condition (5.33), i.e. $\operatorname{Im} \hat{\tilde{\ell}}_{\mathcal{C}_{2}} \leq 0$ is satisfied for $\beta_{0} \geq 1.00025$

REMARK 6. We recognized in this example that Im $\hat{\tilde{\ell}}$ is positive on parts of the unit circle. This means that some modes of the discretized Schrödinger equation could grow very fast. However, we never observed this in our numerical experiments.

We remark that the stability condition of Theorem 5.1 is independent of the 


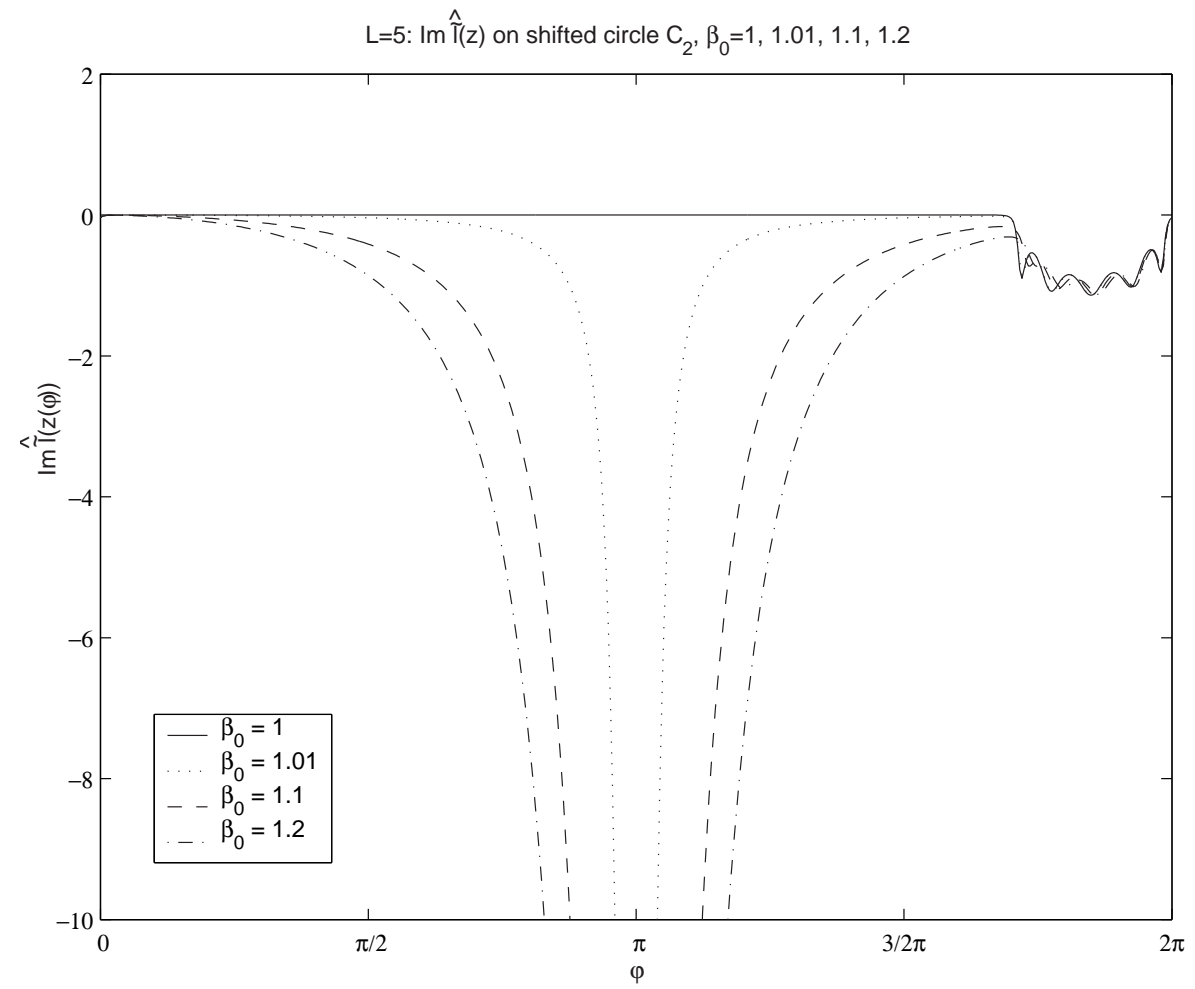

FIG. 5.6. Stability condition (5.33), i.e. Im $\hat{\tilde{\ell}}_{\mathcal{C}_{2}} \leq 0$ for exponential-sum-coefficients with $L=5$. The condition is not satisfied for $\beta_{0}=1$ but is satisfied for $\beta_{0} \geq 1.00025$.

potential $V_{j}$ in the exterior domain:

Proposition 5.2. Let the stability condition (5.27) hold on $\mathcal{C}_{1}^{o}$ for one value of the potential $V_{j} \in \mathbb{R}$. Then this condition holds on the same circle (i.e. for the same $\beta_{0}$ ) for all $V_{j} \in \mathbb{R}$.

Proof. This result in easily seen from the properties of the map $y_{0}$, defined in (5.30): Note that $y_{0}$ maps $\mathcal{C}_{1}^{o}$ onto the half plane $\left\{y \in \mathbb{C} \mid \operatorname{Re} y>\operatorname{Re} y_{0}\left(\beta_{0}\right)\right\}$, independently of $V_{j}$ (cf. Figure 5.3).

$\ell^{2}$-stability in $2 \mathrm{D}$. To finish this section we return to the original $2 \mathrm{D}$-waveguide problem and discuss the stability properties of the complete scheme (1.4) (for $j=$ $1, \ldots, J-1$ ) along with the Dirichlet BCs (1.5) and (exact or approximate) DTBCs for each transversal mode $\psi^{m} ; m=1, \ldots, K-1$ :

$$
\begin{gathered}
\Delta^{+} \hat{\psi}_{0}^{m}(z)=\left(\hat{g}_{0}^{m}(z)-1\right) \hat{\psi}_{0}^{m}(z), \\
\Delta^{-} \hat{\psi}_{J}^{m}(z)=-\left(\hat{g}_{J}^{m}(z)-1\right) \hat{\psi}_{J}^{m}(z) .
\end{gathered}
$$

We recall that the potential $V=V(x, y, t)$ may be $x, y$ and $t$-dependent on the computational domain $[0, X] \times[0, Y]$, but it is assumed to be constant (with the values $V_{ \pm}$) in the leads. Each mode satisfies in the 2 exterior domains a 1D-Schrödinger equation with the effective potential $V_{j}^{m} ; j=0, J$ (cf. (1.7)). If $\hat{g}_{j}(z) ; j=0, J$ are the 
chosen transformed boundary kernels for the free 1D-Schrödinger equation (i.e. with $V_{ \pm}=0$ ), the corresponding kernels for the $2 \mathrm{D}$-modes read

$$
\hat{g}_{j}^{m}\left(z^{*}\right)=\hat{g}_{j}\left(z\left(z^{*}\right)\right)=\hat{g}_{j}\left(\frac{\overline{\tilde{a}} z^{*}+\tilde{b}}{\tilde{a}+\tilde{\tilde{b}} z^{*}}\right),
$$

with $\tilde{a}=4-i \Delta t V_{j}^{m}, \tilde{b}=i \Delta t V_{j}^{m}$ (cf. the derivation of the Transformation rule 3.1).

In fact the stability result of the 1D case easily carries over to 2 dimensions where we shall use the notation

$$
\left\|\psi_{n}\right\|_{2}^{2}=\Delta x \Delta y \sum_{j=1}^{J-1} \sum_{k=1}^{K-1}\left|\psi_{j, k, n}\right|^{2} .
$$

\section{Proposition 5.3.}

(a) Let the transformed boundary kernels $\hat{g}_{j}(z) ; j=0, J$ of the free $1 D$-Schrödinger equation satisfy condition (5.27) for some fixed $\Delta x_{0}$ and $\Delta t_{0}$.

Then the 2D-Schrödinger scheme (1.4) with the BCs (1.5), (5.40) satisfies the following stability estimate:

$$
\left\|\psi_{n}\right\|_{2} \leq\left\|\psi_{0}\right\|_{2} e^{\rho T}, \quad \forall n \Delta t \leq T, 0<\Delta t \leq \Delta t_{0}, \Delta x=\Delta x_{0}, \forall \Delta y>0,
$$

where $\rho=\rho\left(\beta_{0}\right)$ is defined in Theorem 5.1.

(b) Let $\hat{g}_{j}(z) ; j=0, J$ satisfy condition (5.33) for some fixed $\Delta x_{0}$ and $\Delta t_{0}$.

Then, for each fixed $\gamma \geq 1$ and each fixed $\Delta y_{0}>0$, there exists a $\Delta t_{1} \in\left(0, \Delta t_{0}\right]$, such that

$$
\left\|\psi_{n}\right\|_{2} \leq\left\|\psi_{0}\right\|_{2} e^{\rho T}, \quad \forall n \Delta t \leq T, 0<\Delta t \leq \Delta t_{1}, \Delta x=\alpha \Delta t^{\gamma}, \Delta y=\Delta y_{0},
$$

with $\alpha=\Delta x_{0} / \Delta t_{0}^{\gamma}$.

Proof. Since this proof closely follows the strategy of the 1D situation we shall only give the key estimates: Proceeding as in Lemma 5.1 we shall estimate the growth of the new variable

$$
\phi_{j, k, n}:=\psi_{j, k, n} \beta^{-n}
$$

where $\psi_{j, k, n}$ satisfies (1.4), and $\beta=e^{\rho \Delta t}$. Following exactly the steps of (5.17) - (5.21) and summing from $k=1$ to $k=K-1$ yields (with the notation $R_{x}:=4 \Delta x^{2} / \Delta t$, $R_{y}:=4 \Delta y^{2} / \Delta t$, and $\Delta_{y}^{2}$ is the standard second order finite difference operator acting 
in the $y$-direction):

$$
\begin{aligned}
& \sum_{j=1}^{J-1} \sum_{k=1}^{K-1}\left(\left|\phi_{j, k, n+1}\right|^{2}-\left|\phi_{j, k, n}\right|^{2}\right) \\
& \leq \frac{1}{\beta^{2} R_{x}} \operatorname{Im} \sum_{k=1}^{K-1}\left[\left(\bar{\phi}_{0, k, n}+\beta \bar{\phi}_{0, k, n+1}\right) \Delta^{+}\left(\phi_{0, k, n}+\beta \phi_{0, k, n+1}\right)\right. \\
& \left.-\left(\bar{\phi}_{J, k, n}+\beta \bar{\phi}_{J, k, n+1}\right) \Delta^{-}\left(\phi_{J, k, n}+\beta \phi_{J, k, n+1}\right)\right] \\
& +\frac{1}{\beta R_{y}} \operatorname{Im} \sum_{j=1}^{J-1} \sum_{k=1}^{K-1}\left[-\bar{\phi}_{j, k, n} \Delta_{y}^{2}\left(\phi_{j, k, n}+\phi_{j, k, n+1}\right)\right. \\
& \left.\quad+\phi_{j, k, n+1} \Delta_{y}^{2}\left(\bar{\phi}_{j, k, n}+\bar{\phi}_{j, k, n+1}\right)\right] \\
& +\frac{1}{\beta R_{y}} \operatorname{Im} \sum_{j=1}^{J-1} \sum_{k=1}^{K-1}\left[\left(1-\beta^{-1}\right) \bar{\phi}_{j, k, n} \Delta_{y}^{2} \phi_{j, k, n}\right. \\
& \left.\quad+(\beta-1) \phi_{j, k, n+1} \Delta_{y}^{2} \bar{\phi}_{j, k, n+1}\right]
\end{aligned}
$$

Using the Plancherel relation corresponding to the discrete Fourier-sine transform (1.6) we obtain:

$$
\begin{aligned}
& \left\|\phi_{n+1}\right\|_{2}^{2}-\left\|\phi_{n}\right\|_{2}^{2} \\
& \leq \frac{2 K \Delta x \Delta y}{\beta^{2} R_{x}} \operatorname{Im} \sum_{m=1}^{K-1}\left[\left(\bar{\phi}_{0, n}^{m}+\beta \bar{\phi}_{0, n+1}^{m}\right) \Delta^{+}\left(\phi_{0, n}^{m}+\beta \phi_{0, n+1}^{m}\right)\right. \\
& \left.\quad-\left(\bar{\phi}_{J, n}^{m}+\beta \bar{\phi}_{J, n+1}^{m}\right) \Delta^{-}\left(\phi_{J, n}^{m}+\beta \phi_{J, n+1}^{m}\right)\right] \\
& +\frac{4 K \Delta x \Delta y}{\beta R_{y}} \operatorname{Im} \sum_{j=1}^{J-1} \sum_{m=1}^{K-1}\left[-\bar{\phi}_{j, n}^{m}\left(\cos \frac{m \pi}{K}-1\right)\left(\phi_{j, n}^{m}+\phi_{j, n+1}^{m}\right)\right. \\
& \left.\quad+\phi_{j, n+1}^{m}\left(\cos \frac{m \pi}{K}-1\right)\left(\bar{\phi}_{j, n}^{m}+\bar{\phi}_{j, n+1}^{m}\right)\right] \\
& +\frac{4 K \Delta x \Delta y}{\beta R_{y}} \operatorname{Im} \sum_{j=1}^{J-1} \sum_{m=1}^{K-1}\left[\left(1+\beta^{-1}\right) \bar{\phi}_{j, n}^{m}\left(\cos \frac{m \pi}{K}-1\right) \phi_{j, n}^{m}\right. \\
& \left.\quad+(\beta-1) \phi_{j, n+1}^{m}\left(\cos \frac{m \pi}{K}-1\right) \bar{\phi}_{j, n+1}^{m}\right]
\end{aligned}
$$

Note that the last two terms of (5.42) are both zero.

Summing (5.42) from $n=0$ to $n=N$, and using the BCs (5.40) as in (5.22) (5.24) gives:

$$
\begin{aligned}
\left\|\phi_{N+1}\right\|_{2}^{2} \leq\left\|\phi_{0}\right\|_{2}^{2}+ & \frac{\Delta x Y}{\pi \beta^{2} R_{x}} \sum_{m=1}^{K-1} \int_{0}^{2 \pi}\left[\left|\left(1+\beta e^{i \varphi}\right) \hat{\phi}_{0}^{m}\left(e^{i \varphi}\right)\right|^{2} \operatorname{Im}\left(\hat{g}_{0}^{m}\left(\beta e^{i \varphi}\right)-1\right)\right. \\
& \left.+\left|\left(1+\beta e^{i \varphi}\right) \hat{\phi}_{J}^{m}\left(e^{i \varphi}\right)\right|^{2} \operatorname{Im}\left(\hat{g}_{J}^{m}\left(\beta e^{i \varphi}\right)-1\right)\right] d \varphi
\end{aligned}
$$


Next we shall use the assumptions on the transformed boundary kernels $\hat{g}_{j}(z)$ to show that the integral terms of (5.43) are non-positive.

Part (a):

In the assumptions of Part (a) condition (5.27) is imposed on the kernel $\hat{g}_{j}$ of the free 1D Schrödinger equation. By using Proposition 5.2 it implies

$$
\left.\operatorname{Im} \hat{g}_{j}^{m}\left(z, \Delta x_{0}, \Delta t_{0}\right)\right|_{\mathcal{C}_{1}} \leq 0 ; \quad j=0, J ; m=1, \ldots, K-1
$$

for the boundary kernels of each transversal mode $m$. Theorem 5.1 then implies

$$
\operatorname{Im} \hat{g}_{j}^{m}\left(\beta e^{i \varphi}, \Delta x_{0}, \Delta t\right) \leq 0 ; \quad \forall 0<\Delta t \leq \Delta t_{0}, 0 \leq \varphi \leq 2 \pi,
$$

and the assertion follows from

$$
\left\|\psi_{N}\right\|_{2} \beta^{-N}=\left\|\phi_{N}\right\|_{2} \leq\left\|\phi_{0}\right\|_{2}, \quad \forall N \geq 0 .
$$

Part (b):

As before, we have to prove for all modes $m$ :

$$
\operatorname{Im} \hat{g}_{j}^{m}\left(\beta e^{i \varphi}, \alpha \Delta t^{\gamma}, \Delta t\right) \leq 0 ; \quad \forall 0<\Delta t \leq \Delta t_{1}, 0 \leq \varphi \leq 2 \pi .
$$

Following the proof of Theorem 5.2(b), we see that all circles $y_{j}^{m} ; j=0, J ; m=$ $1, \ldots, K-1$ are inside the stability region:

$$
\left\{y_{j}^{m}(\Delta t, \varphi) \mid 0 \leq \varphi \leq 2 \pi\right\} \subset y_{0}\left(\mathcal{C}_{2}^{o}\right)
$$

for $0<\Delta t \leq \Delta t_{1}$, and hence (5.44) follows. Note that such a $\Delta t_{1}>0$ exists, since the effective potentials $V_{j}^{m}$ stay within a finite interval.

\section{Error estimates}

In this section we consider the numerical scheme for the 1D-Schrödinger equation (1.8) with (approximatively) transparent BCs of convolution form (1.17). We shall derive error estimates for the numerical solution when replacing the exact DTBC by an approximation (like those introduced in $\S 3$ ). First we extend the analytic a-priori estimate of Proposition 5.1 for the continuous IBVP for the Schrödinger equation when modifying the BCs. To this end, let $\psi(x, t)$ solve (5.1) with given functions $f_{0}$, $f_{X}$ satisfying (5.3) for some $\alpha_{1} \in \mathbb{R}$.

$\mathbf{H}^{\mathbf{1}}$-a-priori estimate of continuous solution. First we derive an energy estimate for the function $\psi_{x}(x, t)$ :

Proposition 6.1. Assume that $V_{x} \in L_{\text {loc }}^{2}\left(\mathbb{R}_{t}^{+} ; L^{\infty}(0, X)\right), \psi^{I} \in H^{1}(0, X)$. Let the transformed boundary kernels $\hat{f}_{0}, \hat{f}_{X}$ satisfy for some $\alpha_{2} \geq 0$ :

$$
\operatorname{Re}\left\{\left(\overline{\left.s+i V_{-}\right)} \hat{f}_{0}(s)\right\} \geq 0, \quad \operatorname{Re}\left\{\left(\overline{\left.s+i V_{+}\right)} \hat{f}_{X}(s)\right\} \leq 0, \quad s=\alpha_{2}+i \xi,\right.\right.
$$

for all $\xi \in \mathbb{R}$. Then the solution $\psi$ of (5.1) satisfies the a-priori estimate

$$
\left\|\psi_{x}(., t)\right\|_{L^{2}(0, X)}^{2} \leq e^{2 c t}\left[\left\|\psi_{x}^{I}\right\|_{L^{2}(0, X)}^{2}+\left\|\psi^{I}\right\|_{L^{2}(0, X)}^{2} V_{x}^{\max }(t)\right],
$$


where $V_{x}^{\max }(t):=\int_{0}^{t} e^{2\left(\alpha_{1}-\alpha_{2}\right) \tau}\left\|V_{x}(., \tau)\right\|_{L^{\infty}(0, X)}^{2} d \tau$ and $c=\max \left(\alpha_{2}, 1 / 2\right)$.

Proof. A simple energy estimate for $\theta(x, t):=\psi_{x}(x, t) e^{-\alpha_{2} t}, \alpha_{2} \geq 0$, gives

$$
\begin{aligned}
& \frac{d}{d t}\|\theta(., t)\|_{L^{2}(0, X)}^{2}=\left.\operatorname{Im}\left\{\theta(x, t) \bar{\theta}_{x}(x, t)\right\}\right|_{x=0} ^{x=X} \\
& \quad-2 \alpha_{2}\|\theta(., t)\|_{L^{2}(0, X)}^{2}+2 \int_{0}^{X} V_{x}(x, t) \operatorname{Im}\left\{\psi(x, t) e^{-\alpha_{2} t} \bar{\theta}(x, t)\right\} d x .
\end{aligned}
$$

Using the Proposition 5.1 we can estimate the last term:

$$
\begin{aligned}
& 2 \int_{0}^{X} V_{x}(x, t) \operatorname{Im}\left\{\psi(x, t) e^{-\alpha_{2} t} \bar{\theta}(x, t)\right\} d x \\
& \leq 2\|\theta(., t)\|_{L^{2}(0, X)}\left\|\psi(., t) e^{-\alpha_{2} t}\right\|_{L^{2}(0, X)}\left\|V_{x}(., t)\right\|_{L^{\infty}(0, X)} \\
& \leq\|\theta(., t)\|_{L^{2}(0, X)}^{2}+\left\|\psi^{I}\right\|_{L^{2}(0, X)}^{2} e^{2\left(\alpha_{1}-\alpha_{2}\right) t}\left\|V_{x}(., t)\right\|_{L^{\infty}(0, X)}^{2}
\end{aligned}
$$

By integrating in time we obtain from (6.3)

$$
\begin{aligned}
\|\theta(., t)\|_{L^{2}(0, X)}^{2} \leq & \left\|\psi_{x}^{I}\right\|_{L^{2}(0, X)}^{2}+2\left(c-\alpha_{2}\right) \int_{0}^{t}\|\theta(., \tau)\|_{L^{2}(0, X)}^{2} d \tau \\
& +\left\|\psi^{I}\right\|_{L^{2}(0, X)}^{2} \int_{0}^{t} e^{2\left(\alpha_{1}-\alpha_{2}\right) \tau}\left\|V_{x}(., \tau)\right\|_{L^{\infty}(0, X)}^{2} d \tau \\
& +\operatorname{Im} \int_{0}^{t}\left[\bar{\theta}(0, \tau) \theta_{x}(0, \tau)-\bar{\theta}(X, \tau) \theta_{x}(X, \tau)\right] d \tau
\end{aligned}
$$

It remains to show that the last term in (6.4) is negative. We rewrite the two boundary conditions (5.2)

$$
\begin{gathered}
\hat{\psi}_{x}(0, s)=\hat{f}_{0}(s) \hat{\psi}(0, s)=\hat{h}_{0}(s) \hat{\psi}_{x x}(0, s), \quad \hat{h}_{0}(s)=\frac{\hat{f}_{0}(s)}{2\left(V_{-}-i s\right)}, \\
\hat{\psi}_{x}(X, s)=\hat{f}_{X}(s) \hat{\psi}(X, s)=\hat{h}_{X}(s) \hat{\psi}_{x x}(X, s), \quad \hat{h}_{X}(s)=\frac{\hat{f}_{X}(s)}{2\left(V_{+}-i s\right)},
\end{gathered}
$$

$\operatorname{Re} s \geq 0$. Note that $\psi_{x x}$ is not necessarily continuous at $x=0, x=X$. Since the two boundary terms have to be evaluated at $x=0+, x=X-$, the Laplace transformed Schrödinger equation (5.1) was used to replace $\hat{\psi}$ by $\hat{\psi}_{x x}$ in (6.5).

By again denoting the cut-off function by $\chi_{[0, t]}$ we use Plancherel's identity for Laplace transforms (cf. (5.5)). This gives

$$
\begin{aligned}
\operatorname{Im} \int_{0}^{t} \bar{\theta}(0, \tau) \theta_{x}(0, \tau) d \tau & =\operatorname{Im} \int_{0}^{\infty} \overline{\left\{\left(\chi_{[0, t]} \theta_{x}\right) * \tilde{h}_{0}\right\}}(0, \tau) \chi_{[0, t]}(\tau) \theta_{x}(0, \tau) d \tau \\
& =-\left.\frac{1}{2 \pi} \int_{\mathbb{R}} \widehat{\mid \chi_{[0, t]} \theta_{x}}\right|^{2}(0, i \xi) \operatorname{Im} \hat{h}_{0}\left(\alpha_{2}+i \xi\right) d \xi \leq 0
\end{aligned}
$$

provided that $(6.1)$ holds. Here $\tilde{h}_{0}$ is given by $\hat{\tilde{h}}_{0}(s)=\hat{h}_{0}\left(\alpha_{2}+s\right)$. An analogous estimate holds for the boundary term at $x=X$. Finally a Gronwall estimate yields the estimate (6.2).

REMARK 7. Note that the choice of $\hat{h}_{0}(s), \hat{h}_{X}(s)$ in $(6.5)$ is not uncommon; if $\hat{f}_{0}(s)$, $\hat{f}_{X}(s)$ correspond to the TBCs (1.2), (1.3) (cf. Example 5.1) then

$$
\hat{h}_{0}(s)=\frac{e^{\frac{\pi}{4} i}}{\sqrt{2}} \frac{1}{\sqrt[+]{s+i V_{-}}} .
$$


An inverse Laplace transformation yields the impedance boundary condition [34]:

$$
\psi(0, t)=\frac{e^{i \frac{\pi}{4}}}{\sqrt{2 \pi}} \int_{0}^{t} \frac{\psi_{x}(0, \tau) e^{-i V_{-}(t-\tau)}}{\sqrt{t-\tau}} d \tau,
$$

which is equivalent (for smooth functions) to (1.2).

Combining Propositions 5.1 and 6.1 yields an a-priori estimate for the solution at the boundaries (by using a Sobolev-imbedding):

$$
\begin{aligned}
& |\psi(0, t)|^{2}+|\psi(X, t)|^{2} \\
& \quad \leq C\left\{e^{2 \alpha_{1} t}\left\|\psi^{I}\right\|_{L^{2}(0, X)}^{2}+e^{2 c t}\left[\left\|\psi_{x}^{I}\right\|_{L^{2}(0, X)}^{2}+\left\|\psi^{I}\right\|_{L^{2}(0, X)}^{2} V_{x}^{\max }(t)\right]\right\},
\end{aligned}
$$

where $C$ denotes here and in the sequel generic but not necessarily equal constants.

Example 6.1. (exact TBC) We return to the exact TBC of Example 5.1 Its Laplace-transformed kernels $\hat{f}_{0}^{\mathrm{TBC}}, \hat{f}_{X}^{\mathrm{TBC}}$, satisfy the condition (6.1) for all $\alpha_{2} \geq 0$, i.e.

$$
\operatorname{Re}\left\{\left(\overline{s+i V_{-}}\right) \hat{f}_{0}(s)\right\}=\sqrt{2} \operatorname{Re}\left\{e^{-\frac{\pi}{4} i}\left(\overline{s+i V_{-}}\right) \sqrt[+]{s+i V_{-}}\right\} \geq 0, \quad s=\alpha_{2}+i \xi,
$$

holds for all $\xi \in \mathbb{R}$ and analogously for $\hat{f}_{X}^{\mathrm{TBC}}$. This is easily deduced from $\arg (s+$ $\left.i V_{-}\right) \in[-\pi / 2, \pi / 2], \quad$ which implies $\arg \left(\left(\overline{s+i V_{-}}\right) \sqrt[+]{s+i V_{-}}\right) \in$ $[-\pi / 4, \pi / 4]$.

Error estimate of continuous solution. Now let $\mu(x, t)$ solve $(5.1)$ with the exact TBC kernels $f_{0}^{\mathrm{TBC}}, f_{X}^{\mathrm{TBC}}$ (cf. Example 5.1) and let $\psi(x, t)$ solve (5.1) with given functions $f_{0}, f_{X}$ satisfying (5.3) for some $\alpha_{1} \in \mathbb{R}$. Then the error $\kappa(x, t):=$ $\mu(x, t)-\psi(x, t)$ solves the system

$$
\left\{\begin{array}{l}
i \kappa_{t}=-\frac{1}{2} \kappa_{x x}+V(x, t) \kappa, \quad 0<x<X, t>0, \\
\kappa(x, 0)=0, \quad 0<x<X, \\
\hat{\kappa}_{x}(0, s)=\hat{f}_{0}^{\mathrm{TBC}}(s) \hat{\kappa}(0, s)+\left[\hat{f}_{0}^{\mathrm{TBC}}(s)-\hat{f}_{0}(s)\right] \hat{\psi}(0, s), \quad \operatorname{Re} s \geq 0, \\
\hat{\kappa}_{x}(X, s)=\hat{f}_{X}^{\mathrm{TBC}}(s) \hat{\kappa}(X, s)+\left[\hat{f}_{X}^{\mathrm{TBC}}(s)-\hat{f}_{X}(s)\right] \hat{\psi}(X, s), \quad \operatorname{Re} s \geq 0,
\end{array}\right.
$$

and we can formulate the following error estimate:

THEOREM 6.1. Let the assumptions of Proposition 6.1 be fulfilled and let $c=$ $\max \left(\alpha_{2}, 1 / 2\right)$. Then the following estimate holds for all $\alpha \geq 0, \alpha \neq \alpha_{1}, \alpha \neq c$ :

$$
\begin{aligned}
& \|\kappa(., t)\|_{L^{2}(0, X)}^{2} \leq f_{0, X}^{\max }\left[a_{1}\left(e^{2 \alpha_{1} t}-e^{2 \alpha t}\right)+a_{2}\left(e^{2 c t}-e^{2 \alpha t}\right)\right. \\
& \left.\quad+a_{3}\left(e^{2 c t} V_{x}^{\max }(t)-e^{2 \alpha t} \int_{0}^{t} e^{2\left(\alpha_{1}-\alpha_{2}\right) \tau}\left\|V_{x}(., \tau)\right\|_{L^{\infty}(0, X)}^{2} e^{2(c-\alpha) \tau} d \tau\right)\right]
\end{aligned}
$$

with

$$
f_{0, X}^{\max }:=\left\|\hat{f}_{0}^{\mathrm{TBC}}(\alpha+i \xi)-\hat{f}_{0}(\alpha+i \xi)\right\|_{L^{\infty}\left(\mathbb{R}_{\xi}\right)}+\left\|\hat{f}_{X}^{\mathrm{TBC}}(\alpha+i \xi)-\hat{f}_{X}(\alpha+i \xi)\right\|_{L^{\infty}\left(\mathbb{R}_{\xi}\right)}
$$

and

$$
a_{1}=\frac{C\left\|\psi^{I}\right\|_{L^{2}(0, X)}^{2}}{\left(\alpha_{1}-\alpha\right)}, \quad a_{2}=\frac{C\left\|\psi_{x}^{I}\right\|_{L^{2}(0, X)}^{2}}{(c-\alpha)}, \quad a_{3}=\frac{C\left\|\psi^{I}\right\|_{L^{2}(0, X)}^{2}}{(c-\alpha)} .
$$


For $\alpha=\alpha_{1}$ or $\alpha=c$ one takes the obvious limits in (6.10).

Proof. As for Proposition 5.1 the theorem is easily proved by using an energy estimate for the function $\eta(x, t):=\kappa(x, t) e^{-\alpha t}, \alpha \geq 0$, which satisfies:

$$
\left\{\begin{array}{l}
i \eta_{t}=-\frac{1}{2} \eta_{x x}+(V(x, t)-i \alpha) \eta, \quad 0<x<X, t>0, \\
\eta(x, 0)=0, \quad 0<x<X, \\
\hat{\eta}_{x}(0, s)=\hat{f}_{0}^{\mathrm{TBC}}(s+\alpha) \hat{\eta}(0, s)+\left[\hat{f}_{0}^{\mathrm{TBC}}(s+\alpha)-\hat{f}_{0}(s+\alpha)\right] \hat{\psi}(0, s+\alpha), \\
\hat{\eta}_{x}(X, s)=\hat{f}_{X}^{\mathrm{TBC}}(s+\alpha) \hat{\eta}(X, s)+\left[\hat{f}_{X}^{\mathrm{TBC}}(s+\alpha)-\hat{f}_{X}(s+\alpha)\right] \hat{\psi}(X, s+\alpha),
\end{array}\right.
$$

with $\operatorname{Re} s \geq 0$. An energy estimate for $\eta(x, t)$ yields:

$$
\begin{aligned}
\|\eta(., t)\|_{L^{2}(0, X)}^{2}=-2 \alpha \int_{0}^{t} \| & \eta(., \tau) \|_{L^{2}(0, X)}^{2} d \tau \\
& \quad+\operatorname{Im} \int_{0}^{t}\left[\bar{\eta}(0, \tau) \eta_{x}(0, \tau)-\bar{\eta}(X, \tau) \eta_{x}(X, \tau)\right] d \tau .
\end{aligned}
$$

Now Plancherel's identity for Laplace transforms gives

$$
\begin{aligned}
\operatorname{Im} \int_{0}^{t} \bar{\eta}(0, \tau) \eta_{x}(0, \tau) d \tau \\
=\operatorname{Im} \int_{0}^{\infty} \overline{\chi_{[0, t]}(\tau) \eta(0, \tau)}\left\{\left(\chi_{[0, t]} \eta\right) * \tilde{f}_{0}^{\mathrm{TBC}}\right\}(0, \tau) \\
\quad+\left\{\left(\chi_{[0, t]} \psi e^{-\alpha \tau}\right) *\left[\tilde{f}_{0}^{\mathrm{TBC}}-\tilde{f}_{0}\right]\right\}(0, \tau) d \tau \\
=\frac{1}{2 \pi} \int_{\mathbb{R}}\left|\widehat{\chi_{[0, t]} \eta}\right|^{2}(0, i \xi) \operatorname{Im} \hat{f}_{0}^{\mathrm{TBC}}(\alpha+i \xi) \\
\quad+\operatorname{Im}\left[\hat{f}_{0}^{\mathrm{TBC}}(\alpha+i \xi)-\hat{f}_{0}(\alpha+i \xi)\right]\left(\widehat{\chi_{[0, t]} \psi}\right)(0, \alpha+i \xi)\left(\widehat{\chi_{[0, t]} \eta}\right)(0, i \xi) d \xi
\end{aligned}
$$

and analogously for the right boundary term. Here $\hat{\tilde{f}}_{0}$ is given by $\hat{\tilde{f}}_{0}(s)=\hat{f}_{0}(\alpha+s)$. Since $\operatorname{Im} \hat{f}_{0}^{\mathrm{TBC}}(\alpha+i \xi) \leq 0$ and $\operatorname{Im} \hat{f}_{X}^{\mathrm{TBC}}(\alpha+i \xi) \geq 0$ for all $\alpha \geq 0, \xi \in \mathbb{R}$ (cf. Example 5.1) we obtain

$$
\begin{aligned}
& \|\eta(., t)\|_{L^{2}(0, X)}^{2} \\
& \quad \leq\left\|\hat{f}_{0}^{\mathrm{TBC}}(\alpha+i \xi)-\hat{f}_{0}(\alpha+i \xi)\right\|_{L^{\infty}\left(\mathbb{R}_{\xi}\right)}\|\eta(0, \tau)\|_{L^{2}(0, t)}\left\|\psi(0, \tau) e^{-\alpha \tau}\right\|_{L^{2}(0, t)} \\
& \quad+\left\|\hat{f}_{X}^{\mathrm{TBC}}(\alpha+i \xi)-\hat{f}_{X}(\alpha+i \xi)\right\|_{L^{\infty}\left(\mathbb{R}_{\xi}\right)}\|\eta(X, \tau)\|_{L^{2}(0, t)}\left\|\psi(X, \tau) e^{-\alpha \tau}\right\|_{L^{2}(0, t)} .
\end{aligned}
$$

It only remains to estimate the above boundary terms. Using the estimate (6.7) we obtain

$$
\begin{aligned}
|\eta(0, t)|^{2} & =e^{-2 \alpha t}|\mu(0, t)-\psi(0, t)|^{2} \\
& \leq C e^{-2 \alpha t}\left\{e^{2 \alpha_{1} t}\left\|\psi^{I}\right\|_{L^{2}(0, X)}^{2}+e^{2 c t}\left[\left\|\psi_{x}^{I}\right\|_{L^{2}(0, X)}^{2}+\left\|\psi^{I}\right\|_{L^{2}(0, X)}^{2} V_{x}^{\max }(t)\right]\right\}
\end{aligned}
$$

and analogously for $|\eta(X, t)|^{2}$. Integrating in time yields

$$
\begin{aligned}
& \|\eta(0, .)\|_{L^{2}(0, t)}^{2} \leq a_{1}\left(e^{2\left(\alpha_{1}-\alpha\right) t}-1\right)+a_{2}\left(e^{2(c-\alpha) t}-1\right) \\
& \quad+a_{3}\left(e^{2(c-\alpha) t} V_{x}^{\max }(t)-\int_{0}^{t} e^{2\left(\alpha_{1}-\alpha_{2}\right) \tau}\left\|V_{x}(., \tau)\right\|_{L^{\infty}(0, X)}^{2} e^{2(c-\alpha) \tau} d \tau\right)
\end{aligned}
$$


and an estimate of the same form holds for $\left\|\psi(X, \tau) e^{-\alpha \tau}\right\|_{L^{2}(0, t)}$, i.e. we obtain the estimate (6.10).

To illustrate this result we now consider two simple examples:

Example 6.2. ("cut-off" TBC) We continue the discussion of Example 5.2 with the approximate $\mathrm{BC}-$ kernel

$$
f_{0}(t)=f_{0}^{\mathrm{TBC}}(t) H(T-t) .
$$

Analogously to the procedure in Example 5.2 one can verify numerically that its Laplace transform

$$
\hat{f}_{0}(s)=\sqrt{2} e^{-\frac{\pi}{4} i} \sqrt[+]{s+i V_{-}}+\frac{1}{\sqrt{2 \pi}} e^{-\frac{\pi}{4} i} \int_{T}^{\infty} t^{-\frac{3}{2}} e^{-s t} d t, \quad s=\alpha_{2}+i \xi, \xi \in \mathbb{R},
$$

satisfies the condition (6.1) (with $V_{-}=0$ ) for $\tilde{\alpha}_{2} \geq 0.25$ if $T=1$.

Next we verify that the two kernels $f_{0}^{\mathrm{TBC}}$ and $f_{0}$ satisfy an error estimate like those appearing on the right hand side of (6.10). These two kernels satisfy

$$
\left\|f_{0}^{\mathrm{TBC}}-f_{0}\right\|_{L^{1}(0, \infty)}=\sqrt{\frac{2}{\pi T}},
$$

and hence

$$
\left\|\hat{f}_{0}^{\mathrm{TBC}}(\alpha+i \xi)-\hat{f}_{0}(\alpha+i \xi)\right\|_{L^{\infty}\left(\mathbb{R}_{\xi}\right)} \leq \sqrt{\frac{2}{\pi T}} e^{-\alpha T}
$$

holds for all $\alpha \geq 0$.

Example 6.3. (rational function kernel) As a second example assume now that $\hat{f}_{0}$ is a rational function (as proposed in $[3,13,17]$ ):

$$
\hat{f}_{0}(s)=\frac{P_{L-1}(s)}{Q_{L}(s)} .
$$

Since $\hat{f}_{0}^{\mathrm{TBC}}(s)=\sqrt{2} e^{-\frac{\pi}{4} i} \sqrt[+]{s}$ and $\hat{f}_{0}(s)$ have different asymptotic behaviours for large $|s|$, we conclude

$$
\hat{f}_{0}^{\mathrm{TBC}}(\alpha+i \xi)-\hat{f}_{0}(\alpha+i \xi) \notin L^{\infty}\left(\mathbb{R}_{\xi}\right)
$$

for any $\alpha \geq 0$. Hence Theorem 6.1 does not apply. We remark that this difficulty will not arise for the discrete BCs with exponential-sum-coefficients derived in $\S 3$ (cf. Example 6.5).

$\mathbf{h}^{\mathbf{1}}$-a-priori estimate of discrete solution. Analogously to the continuous case let $\psi_{j, n}$ solve the Crank-Nicolson scheme (5.10) with $\hat{g}_{0}, \hat{g}_{J}$ satisfying (5.15) for some $\beta=\beta_{1} \geq 1$. From now on we shall assume that $V_{j}=V_{-}$for $j \leq 1$ and $V_{j}=V_{+}$ for $j \geq J-1$ in addition to the assumptions in $\S 5$. First we derive a discrete $h^{1}-$ estimate of the solution of (5.10) which is the discrete analogue of Proposition 6.1:

Proposition 6.2. Let the transformed boundary transfer functions $\hat{g}_{0}, \hat{g}_{J}$ satisfy the following condition for some (sufficiently large) $\beta_{2} \geq 1$ :

$$
\operatorname{Re}\left\{\bar{y}_{1}\left(\beta_{2} e^{i \varphi}\right)\left[1-\frac{1}{\hat{g}_{0}\left(\beta_{2} e^{i \varphi}\right)}\right]\right\} \geq 0, \quad 0 \leq \varphi \leq 2 \pi,
$$




$$
\operatorname{Re}\left\{\bar{y}_{J-1}\left(\beta_{2} e^{i \varphi}\right)\left[1-\frac{1}{\hat{g}_{J}\left(\beta_{2} e^{i \varphi}\right)}\right]\right\} \geq 0, \quad 0 \leq \varphi \leq 2 \pi,
$$

with

$$
y_{j}(z)=\frac{R}{2}\left(\frac{z-1}{z+1}+i \nu_{j}\right), \quad \nu_{1}=\frac{\Delta t V_{-}}{2}, \quad \nu_{J-1}=\frac{\Delta t V_{+}}{2} .
$$

Assume also that $\hat{g}_{0}, \hat{g}_{J}$ are analytic for $|z| \geq \beta_{2}$. Then the following estimate holds for $\Delta t<2, n \in \mathbb{N}$ :

$$
\left\|\Delta^{+} \psi_{n}\right\|_{2, *}^{2} \leq c^{2 n}\left[\left\|\Delta^{+} \psi_{0}\right\|_{2, *}^{2}+\frac{\left(1+\beta_{1}\right)^{2}}{2 \beta_{2}^{2}} \frac{\left\|\psi_{0}\right\|_{2}^{2}}{2-\Delta t} V_{\Delta^{+}, n-1}^{\max }\right],
$$

where

$$
V_{\Delta^{+}, n}^{\max }:=\Delta t \sum_{k=0}^{n}\left(\frac{\beta_{1}}{\beta_{2}}\right)^{2 k}\left\|\Delta^{+} V_{k+\frac{1}{2}}\right\|_{\infty}^{2}, \quad c=\max \left(\beta_{2}, \sqrt{\frac{2+\Delta t}{2-\Delta t}}\right)
$$

and the discrete norms are defined by

$$
\left\|\Delta^{+} \psi_{n}\right\|_{2, *}^{2}=\Delta x \sum_{j=1}^{J-2}\left|\Delta^{+} \psi_{j, n}\right|^{2}, \quad\left\|\Delta^{+} V_{n+\frac{1}{2}}\right\|_{\infty}=\max _{j=1, \ldots, J-2}\left|\Delta^{+} V_{j, n+\frac{1}{2}}\right| .
$$

(Note the difference to the discrete $L^{2}{ }^{-}$norm in (5.14).)

Proof. The proof is based on a discrete energy estimate for the new variable

$$
\theta_{j, n}:=\beta_{2}^{-n} \Delta^{+} \psi_{j, n}
$$

which solves the equation

$$
\begin{aligned}
-i R\left(\theta_{j, n+1}-\theta_{j, n}\right)= & \left(\Delta^{2}-w V_{j, n+\frac{1}{2}}\right)\left(\theta_{j, n+1}+\theta_{j, n}\right) \\
& +\left(\beta_{2}^{-1}-1\right)\left(\Delta^{2}-w V_{j, n+\frac{1}{2}}-i R\right) \theta_{j, n} \\
& -w \beta_{2}^{-n-1}\left(\psi_{j+1, n+1}+\psi_{j+1, n}\right) \Delta^{+} V_{j, n+\frac{1}{2}} .
\end{aligned}
$$

Alternatively this can be written as

$$
\begin{aligned}
-i R\left(\theta_{j, n+1}-\theta_{j, n}\right)= & \left(\Delta^{2}-w V_{j, n+\frac{1}{2}}\right)\left(\theta_{j, n+1}+\theta_{j, n}\right) \\
& +\left(\beta_{2}-1\right)\left(\Delta^{2}-w V_{j, n+\frac{1}{2}}+i R\right) \theta_{j, n+1} \\
& -w \beta_{2}^{-n}\left(\psi_{j+1, n+1}+\psi_{j+1, n}\right) \Delta^{+} V_{j, n+\frac{1}{2}}
\end{aligned}
$$

for $j=1, \ldots, J-2$ together with the initial condition

$$
\theta_{j, 0}=\Delta^{+} \psi_{j, 0}, \quad j=1, \ldots, J-2,
$$

and the two transformed boundary conditions

$$
\begin{aligned}
\hat{\theta}_{0}(z) & =\left[\hat{g}_{0}\left(\beta_{2} z\right)-1\right] \hat{\psi}_{0}\left(\beta_{2} z\right), \\
\hat{\theta}_{J-1}(z) & =-\left[\hat{g}_{J}\left(\beta_{2} z\right)-1\right] \hat{\psi}_{J}\left(\beta_{2} z\right) .
\end{aligned}
$$


We multiply equation (6.17a) with $\bar{\theta}_{j, n}$ and the complex conjugate of $(6.17 \mathrm{~b})$ with $-\theta_{j, n+1}$ and sum it up for $j=1, \ldots, J-2$. After a lengthy calculation one obtains the following formula:

$$
\begin{aligned}
\left\|\theta_{n+1}\right\|_{2, *}^{2}-\left\|\theta_{n}\right\|_{2, *}^{2}= & \frac{\Delta t}{4 \beta_{2}^{2} \Delta x} \operatorname{Im}\left[\left(\bar{\theta}_{0, n}+\beta_{2} \bar{\theta}_{0, n+1}\right) \Delta^{+}\left(\theta_{0, n}+\beta_{2} \theta_{0, n+1}\right)\right. \\
& \left.-\left(\bar{\theta}_{J-1, n}+\beta_{2} \bar{\theta}_{J-1, n+1}\right) \Delta^{-}\left(\theta_{J-1, n}+\beta_{2} \theta_{J-1, n+1}\right)\right] \\
& -\left(1-\beta_{2}^{-2}\right)\left\|\theta_{n}\right\|_{2, *}^{2} \\
& +\frac{\Delta t \Delta x}{2 \beta_{2}^{n+2}} \sum_{j=1}^{J-2} \operatorname{Im}\left[\left(\bar{\theta}_{j, n}+\beta_{2} \bar{\theta}_{j, n+1}\right)\left(\psi_{j+1, n+1}+\psi_{j+1, n}\right)\right] \Delta^{+} V_{j, n+\frac{1}{2}}
\end{aligned}
$$

which is the discrete analogue of (6.3). We estimate the last term using Lemma 5.1

$$
\begin{aligned}
\Delta x \sum_{j=1}^{J-2} \operatorname{Im}\left[\left(\bar{\theta}_{j, n}+\beta_{2} \bar{\theta}_{j, n+1}\right) \beta_{2}^{-n}\left(\psi_{j+1, n+1}+\psi_{j+1, n}\right)\right] \Delta^{+} V_{j, n+\frac{1}{2}} \\
\quad \leq\left\|\theta_{n}+\beta_{2} \theta_{n+1}\right\|_{2, *} \beta_{2}^{-n}\left\|\psi_{n+1}+\psi_{n}\right\|_{2}\left\|\Delta^{+} V_{n+\frac{1}{2}}\right\|_{\infty} \\
\quad \leq\left\|\theta_{n}+\beta_{2} \theta_{n+1}\right\|_{2, *}\left(1+\beta_{1}\right)\left\|\psi_{0}\right\|_{2}\left(\frac{\beta_{1}}{\beta_{2}}\right)^{n}\left\|\Delta^{+} V_{n+\frac{1}{2}}\right\|_{\infty} \\
\quad \leq\left\|\theta_{n}\right\|_{2, *}^{2}+\beta_{2}^{2}\left\|\theta_{n+1}\right\|_{2, *}^{2}+\frac{\left(1+\beta_{1}\right)^{2}}{2}\left\|\psi_{0}\right\|_{2}^{2}\left(\frac{\beta_{1}}{\beta_{2}}\right)^{2 n}\left\|\Delta^{+} V_{n+\frac{1}{2}}\right\|_{\infty}^{2}
\end{aligned}
$$

and we obtain

$$
\begin{aligned}
\left\|\theta_{n+1}\right\|_{2, *}^{2}-\left\|\theta_{n}\right\|_{2, *}^{2} \leq & \left(\beta_{2}^{-2}-1+\frac{\Delta t}{2 \beta_{2}^{2}}\right)\left\|\theta_{n}\right\|_{2, *}^{2}+\frac{\Delta t}{2}\left\|\theta_{n+1}\right\|_{2, *}^{2} \\
& +\frac{\Delta t}{4 \beta_{2}^{2}}\left(1+\beta_{1}\right)^{2}\left\|\psi_{0}\right\|_{2}^{2}\left(\frac{\beta_{1}}{\beta_{2}}\right)^{2 n}\left\|\Delta^{+} V_{n+\frac{1}{2}}\right\|_{\infty}^{2} \\
& +\frac{\Delta t}{4 \beta_{2}^{2} \Delta x} \operatorname{Im}\left[\left(\bar{\theta}_{0, n}+\beta_{2} \bar{\theta}_{0, n+1}\right) \Delta^{+}\left(\theta_{0, n}+\beta_{2} \theta_{0, n+1}\right)\right. \\
& \left.\quad-\left(\bar{\theta}_{J-1, n}+\beta_{2} \bar{\theta}_{J-1, n+1}\right) \Delta^{-}\left(\theta_{J-1, n}+\beta_{2} \theta_{J-1, n+1}\right)\right] .
\end{aligned}
$$

Summing for $n=0, \ldots, N$ yields

$$
\begin{aligned}
\left(1-\frac{\Delta t}{2}\right)\left\|\theta_{N+1}\right\|_{2, *}^{2} \leq & -\left(1-\frac{\Delta t}{2}\right) \sum_{n=1}^{N}\left\|\theta_{n}\right\|_{2, *}^{2}+\left(1+\frac{\Delta t}{2}\right) \beta_{2}^{-2} \sum_{n=0}^{N}\left\|\theta_{n}\right\|_{2, *}^{2} \\
& +\frac{\left(1+\beta_{1}\right)^{2}}{4 \beta_{2}^{2}}\left\|\psi_{0}\right\|_{2}^{2} V_{\Delta^{+}, N}^{\max } \\
& +\frac{\Delta t}{4 \beta_{2}^{2} \Delta x} \operatorname{Im} \sum_{n=0}^{N}\left[\left(\bar{\theta}_{0, n}+\beta_{2} \bar{\theta}_{0, n+1}\right) \Delta^{+}\left(\theta_{0, n}+\beta_{2} \theta_{0, n+1}\right)\right. \\
& \left.\quad-\left(\bar{\theta}_{J-1, n}+\beta_{2} \bar{\theta}_{J-1, n+1}\right) \Delta^{-}\left(\theta_{J-1, n}+\beta_{2} \theta_{J-1, n+1}\right)\right] .
\end{aligned}
$$

It remains to determine the sign of the last term in (6.19). To this end, we rewrite 
the two boundary conditions (6.18):

$$
\begin{aligned}
\hat{\theta}_{0}(z) & =\left[1-\frac{1}{\hat{g}_{0}\left(\beta_{2} z\right)}\right] \hat{\psi}_{1}\left(\beta_{2} z\right)=\hat{\tilde{h}}_{0}(z) \Delta^{+} \hat{\theta}_{0}(z), \\
\hat{\theta}_{J-1}(z) & =-\left[1-\frac{1}{\hat{g}_{J}\left(\beta_{2} z\right)}\right] \hat{\psi}_{J-1}\left(\beta_{2} z\right)=\hat{\tilde{h}}_{J}(z) \Delta^{-} \hat{\theta}_{J-1}(z),
\end{aligned}
$$

with

$$
\hat{\tilde{h}}_{0}(z)=\frac{1-\frac{1}{\hat{g}_{0}\left(\beta_{2} z\right)}}{-i R \frac{\beta_{2} z-1}{\beta_{2} z+1}+w V_{-}}, \quad \hat{\tilde{h}}_{J}(z)=\frac{-\left[1-\frac{1}{\hat{g}_{J}\left(\beta_{2} z\right)}\right]}{-i R \frac{\beta_{2} z-1}{\beta_{2} z+1}+w V_{+}} .
$$

As in Section 5 we define (for $N$ fixed) the two sequences,

$$
\begin{gathered}
u_{n}:= \begin{cases}\Delta^{+}\left(\theta_{0, n}+\beta_{2} \theta_{0, n+1}\right), & n=0, \ldots, N, \\
0, & n>N,\end{cases} \\
v_{n}:=u_{n} * \tilde{h}_{0, n}, \quad n \in \mathbb{N}_{0}, \quad \tilde{h}_{0, n}=\mathcal{Z}^{-1}\left\{\hat{\tilde{h}}_{0}(z)\right\} .
\end{gathered}
$$

Now using Plancherel's Theorem for $Z$-transforms we can show that

$$
\begin{aligned}
& \operatorname{Im} \sum_{n=0}^{N}\left[\left(\bar{\theta}_{0, n}+\beta_{2} \bar{\theta}_{0, n+1}\right) \Delta^{+}\left(\theta_{0, n}+\beta_{2} \theta_{0, n+1}\right)\right] \\
& \quad=\operatorname{Im} \sum_{n=0}^{N} \bar{v}_{n} u_{n}=-\operatorname{Im} \sum_{n=0}^{\infty} v_{n} \bar{u}_{n}=-\frac{1}{2 \pi} \int_{0}^{2 \pi}\left|\hat{u}\left(e^{i \varphi}\right)\right|^{2} \operatorname{Im} \hat{\tilde{h}}_{0}\left(e^{i \varphi}\right) d \varphi
\end{aligned}
$$

is negative since

$$
\operatorname{Im} \hat{\tilde{h}}_{0}\left(e^{i \varphi}\right)=\operatorname{Im} \frac{i}{2} \frac{1-\frac{1}{\hat{g}_{0}\left(\beta_{2} e^{i \varphi}\right)}}{y_{1}\left(\beta_{2} e^{i \varphi}\right)}=\frac{\operatorname{Re}\left\{\bar{y}_{1}\left(\beta_{2} e^{i \varphi}\right)\left[1-\frac{1}{\hat{g}_{0}\left(\beta_{2} e^{i \varphi}\right)}\right]\right\}}{2\left|y_{1}\left(\beta_{2} e^{i \varphi}\right)\right|^{2}} \geq 0
$$

due to the assumption (6.14). An analogous estimate holds for the right boundary term.

Finally (6.19) yields for $\Delta t<2$

$$
\left\|\theta_{N+1}\right\|_{2, *}^{2} \leq\left\|\theta_{0}\right\|_{2, *}^{2}+\frac{\left(1+\beta_{1}\right)^{2}}{2 \beta_{2}^{2}} \frac{\left\|\psi_{0}\right\|_{2}^{2}}{2-\Delta t} V_{\Delta^{+}, N}^{\max }+\left(\frac{2+\Delta t}{2-\Delta t} \beta_{2}^{-2}-1\right) \sum_{n=0}^{N}\left\|\theta_{n}\right\|_{2, *}^{2} .
$$

With the discrete Gronwall-type estimate [35, Lemma 1.4.2] for the function $\left\|\theta_{N+1}\right\|_{2, *}^{2}$ we obtain:

$$
\left\|\theta_{N+1}\right\|_{2, *}^{2} \leq\left(\frac{2+\Delta t}{2-\Delta t} \beta_{2}^{-2}\right)^{N+1}\left[\left\|\theta_{0}\right\|_{2, *}^{2}+\frac{\left(1+\beta_{1}\right)^{2}}{2 \beta_{2}^{2}} \frac{\left\|\psi_{0}\right\|_{2}^{2}}{2-\Delta t} V_{\Delta^{+}, N}^{\max }\right]
$$

provided that $(2+\Delta t) /(2-\Delta t) \geq \beta_{2}^{2}$. This yields the estimate (6.16).

By combining Lemma 5.1 and Proposition 6.2 and using the discrete Sobolevinequality

$$
\left|\psi_{j, n}\right|^{2} \leq C(X)\left\{\left\|\psi_{n}\right\|_{2}^{2}+(\Delta x)^{-2}\left\|\Delta^{+} \psi_{n}\right\|_{2, *}^{2}\right\}, \quad j=1, \ldots, J-1,
$$


we now obtain an a-priori pointwise estimate for the discrete solution:

$$
\left|\psi_{j, n}\right|^{2} \leq C(X)\left\{\beta_{1}^{2 n}\left\|\psi_{0}\right\|_{2}^{2}+c^{2 n}(\Delta x)^{-2}\left[\left\|\Delta^{+} \psi_{0}\right\|_{2}^{2}+\frac{\left(1+\beta_{1}\right)^{2}}{2 \beta_{2}^{2}} \frac{\left\|\psi_{0}\right\|_{2}^{2}}{2-\Delta t} V_{\Delta^{+}, n-1}^{\max }\right]\right\}
$$

for $j=1, \ldots, J-1, n \in \mathbb{N}$. Note that this is a discrete analogue of (6.7).

Example 6.4. (exact DTBC) We consider the exact DTBC of Example 5.3. The $Z$-transformed boundary kernel

$$
\hat{g}_{0}^{\mathrm{TBC}}(z)=\hat{\ell}_{0}(z)=1-i y_{1}(z)+\sqrt{-y_{1}(z)\left(y_{1}(z)+2 i\right)}
$$

(with the branch of the square root chosen such that $\left|\hat{\ell}_{0}(z)\right| \geq 1$ ) satisfies the condition (6.14) for all $\beta_{2} \geq 1$. To verify this, one has to check that

$$
\operatorname{Re}\left\{\bar{y}_{1}\left[i y_{1}+\sqrt{-y_{1}\left(y_{1}+2 i\right)}\right]\right\}=\operatorname{Re}\left\{\bar{y}_{1} \sqrt{-y_{1}(z)\left(y_{1}+2 i\right)}\right\} \geq 0
$$

holds for all $y_{1}=y_{1}(z)$ with $\operatorname{Re} y_{1} \geq 0$ (cf. Figure 5.3): For $\beta_{2}=1$ (i.e. Re $y_{1}=0$ ) this is easily done analytically, and for $\beta_{2}>1$ one can do it numerically. Note that (6.26) is the analogue of (6.8) for the exact DTBC.

Error estimate of discrete solution. Now let $\mu_{j, n}$ be the solution of (5.10) with the exact TBC kernels $\hat{g}_{0}^{\mathrm{TBC}}=\hat{\ell}_{0}, \hat{g}_{J}^{\mathrm{TBC}}=\hat{\ell}_{J}$ (cf. (1.10)) and let $\psi_{j, n}$ solve the Crank-Nicolson scheme (5.10) with other transformed kernels $\hat{g}_{0}, \hat{g}_{J}$ satisfying (5.15) for some $\beta_{1} \geq 1$. Then the discrete error $\kappa_{j, n}:=\mu_{j, n}-\psi_{j, n}$ solves

$$
\left\{\begin{aligned}
-i R\left(\kappa_{j, n+1}-\kappa_{j, n}\right) & =\Delta^{2}\left(\kappa_{j, n+1}+\kappa_{j, n}\right)-w V_{j, n+\frac{1}{2}}\left(\kappa_{j, n+1}+\kappa_{j, n}\right), \\
& j=1, \ldots, J-1, \\
\kappa_{j, 0} & =0, \quad j=0, \ldots, J, \\
\Delta^{+} \hat{\kappa}_{0}(z) & =\left[\hat{g}_{0}^{\mathrm{TBC}}(z)-1\right] \hat{\kappa}_{0}(z)+\left[\hat{g}_{0}^{\mathrm{TBC}}(z)-\hat{g}_{0}(z)\right] \hat{\psi}_{0}(z), \\
\Delta^{-} \hat{\kappa}_{J}(z) & =-\left[\hat{g}_{J}^{\mathrm{TBC}}(z)-1\right] \hat{\kappa}_{J}(z)-\left[\hat{g}_{J}^{\mathrm{TBC}}(z)-\hat{g}_{J}(z)\right] \hat{\psi}_{J}(z),
\end{aligned}\right.
$$

and the following estimate can be proved:

TheOREm 6.2. Let the assumptions of Proposition 6.2 be fulfilled and assume $\Delta t<$ 2. Then the following error estimate holds for all $\beta \geq 1, \beta \neq \beta_{1}, \beta \neq c=$ $\max \left(\beta_{2}, \sqrt{(2+\Delta t) /(2-\Delta t)}\right)$

$$
\begin{gathered}
\left\|\kappa_{n}\right\|_{2}^{2} \leq \frac{g_{0, J}^{\max }}{2} \\
\cdot \Delta t(1+\beta)^{2}\left\{\frac{\beta^{2 n}-\beta_{1}^{2 n}}{\beta^{2}-\beta_{1}^{2}}\left\|\psi_{0}\right\|_{2}^{2}+\frac{\beta^{2 n}-c^{2 n}}{\beta^{2}-c^{2}} X\left\|\Delta^{+} \psi_{0}\right\|_{2}^{2}\right. \\
\left.+X \frac{\left(1+\beta_{1}\right)^{2}}{2 \beta_{2}^{2}} \frac{\left\|\psi_{0}\right\|_{2}^{2}}{2-\Delta t} \beta^{2(n-1)} \sum_{k=0}^{n-1}\left(\frac{c}{\beta}\right)^{2 k} V_{\Delta^{+}, k-1}^{\max }\right\},
\end{gathered}
$$

with

$$
\begin{aligned}
g_{0, J}^{\max }:=\frac{1}{\Delta x}( & \left\|\frac{1}{\hat{g}_{0}\left(\beta e^{i \varphi}\right)}-\frac{1}{\hat{g}_{0}^{\mathrm{TBC}}\left(\beta e^{i \varphi}\right)}\right\|_{L^{\infty}(0,2 \pi)} \\
& \left.+\left\|\frac{1}{\hat{g}_{J}\left(\beta e^{i \varphi}\right)}-\frac{1}{\hat{g}_{J}^{\mathrm{TBC}}\left(\beta e^{i \varphi}\right)}\right\|_{L^{\infty}(0,2 \pi)}\right) .
\end{aligned}
$$


For $\beta=\beta_{1}$ or $\beta=c$ we obtain the estimate by obvious limits.

Proof. The new discrete variable $\eta_{j, n}:=\kappa_{j, n} \beta^{-n}, \beta \geq 1$, satisfies the two equations

$$
\begin{aligned}
-i R\left(\eta_{j, n+1}-\eta_{j, n}\right)= & \Delta^{2}\left(\eta_{j, n+1}+\eta_{j, n}\right)-w V_{j, n+\frac{1}{2}}\left(\eta_{j, n+1}+\eta_{j, n}\right) \\
& +\left(\beta^{-1}-1\right)\left(\Delta^{2}-w V_{j, n+\frac{1}{2}}-i R\right) \eta_{j, n}, \\
-i R\left(\eta_{j, n+1}-\eta_{j, n}\right)= & \Delta^{2}\left(\eta_{j, n+1}+\eta_{j, n}\right)-w V_{j, n+\frac{1}{2}}\left(\eta_{j, n+1}+\eta_{j, n}\right) \\
& +(\beta-1)\left(\Delta^{2}-w V_{j, n+\frac{1}{2}}+i R\right) \eta_{j, n+1},
\end{aligned}
$$

for $j=1, \ldots, J-1$ and the two boundary conditions

$$
\begin{aligned}
\Delta^{+} \hat{\eta}_{0}(z) & =\left[\hat{g}_{0}^{\mathrm{TBC}}(\beta z)-1\right] \hat{\eta}_{0}(z)+\left[\hat{g}_{0}^{\mathrm{TBC}}(\beta z)-\hat{g}_{0}(\beta z)\right] \hat{\psi}_{0}(\beta z), \\
\Delta^{-} \hat{\eta}_{J}(z) & =-\left[\hat{g}_{J}^{\mathrm{TBC}}(\beta z)-1\right] \hat{\eta}_{J}(z)-\left[\hat{g}_{J}^{\mathrm{TBC}}(\beta z)-\hat{g}_{J}(\beta z)\right] \hat{\psi}_{J}(\beta z) .
\end{aligned}
$$

We multiply equation (6.29a) with $\bar{\eta}_{j, n}$, the complex conjugate of (6.29b) with $\eta_{j, n+1}$ and sum it up for $j=1, \ldots, J-1$. This gives finally

$$
\begin{aligned}
-i R \sum_{j=1}^{J-1}\left|\eta_{j, n+1}\right|^{2}-\left|\eta_{j, n}\right|^{2} & =\sum_{j=1}^{J-1} \bar{\eta}_{j, n} \Delta^{2}\left(\eta_{j, n+1}+\eta_{j, n}\right)-\sum_{j=1}^{J-1} \eta_{j, n+1} \Delta^{2}\left(\bar{\eta}_{j, n+1}+\bar{\eta}_{j, n}\right) \\
& +\left(\beta^{-1}-1\right) \sum_{j=1}^{J-1} \bar{\eta}_{j, n} \Delta^{2} \eta_{j, n}-(\beta-1) \sum_{j=1}^{J-1} \eta_{j, n+1} \Delta^{2} \bar{\eta}_{j, n+1} \\
& -\left(\beta^{-1}-1\right) i R \sum_{j=1}^{J-1}\left|\eta_{j, n}\right|^{2}+(\beta-1) i R \sum_{j=1}^{J-1}\left|\eta_{j, n+1}\right|^{2} \\
& +w \sum_{j=1}^{J-1} V_{j, n+\frac{1}{2}}\left(\beta\left|\eta_{j, n+1}\right|^{2}-\beta^{-1}\left|\eta_{j, n}\right|^{2}\right) .
\end{aligned}
$$

After a lengthy calculation one obtains the following expression for the discrete $L^{2}$ norm of the error:

$$
\begin{aligned}
\left\|\eta_{N+1}\right\|_{2}^{2}= & \frac{1-\beta^{2}}{\beta^{2}} \sum_{n=1}^{N}\left\|\eta_{n}\right\|_{2}^{2} \\
& +\frac{\Delta t}{4 \beta^{2} \Delta x} \operatorname{Im} \sum_{n=0}^{N}\left[\left(\bar{\eta}_{0, n}+\beta \bar{\eta}_{0, n+1}\right) \Delta^{+}\left(\eta_{0, n}+\beta \eta_{0, n+1}\right)\right. \\
& \left.\quad-\left(\bar{\eta}_{J, n}+\beta \bar{\eta}_{J, n+1}\right) \Delta^{-}\left(\eta_{J, n}+\beta \eta_{J, n+1}\right)\right] .
\end{aligned}
$$

Since $\beta \geq 1$ it only remains to estimate the boundary terms in (6.31). To this end, we rewrite the two boundary conditions (6.30):

$$
\begin{aligned}
\Delta^{-} \hat{\eta}_{1}(z) & =\hat{\tilde{h}}_{0}(z) \hat{\eta}_{1}(z)+\hat{\tilde{k}}_{0}(z) \hat{\psi}_{1}(\beta z), \\
\Delta^{+} \hat{\eta}_{J-1}(z) & =-\hat{\tilde{h}}_{J}(z) \hat{\eta}_{J-1}(z)-\hat{\tilde{k}}_{J}(z) \hat{\psi}_{J-1}(\beta z) .
\end{aligned}
$$

with

$$
\hat{\tilde{h}}_{j}(z)=1-\frac{1}{\hat{g}_{j}^{\mathrm{TBC}}(\beta z)}, \quad \hat{\tilde{k}}_{j}(z)=\frac{1}{\hat{g}_{j}(\beta z)}-\frac{1}{\hat{g}_{j}^{\mathrm{TBC}}(\beta z)}, \quad j=0, J .
$$


Again we define (for $N$ fixed) the three sequences,

$$
u_{n}:=\left\{\begin{array}{ll}
\eta_{1, n}+\beta \eta_{1, n+1}, & n=0, \ldots, N, \\
0, & n>N,
\end{array} w_{n}:= \begin{cases}\psi_{1, n}+\beta \psi_{1, n+1}, & n=0, \ldots, N, \\
0, & n>N,\end{cases}\right.
$$

and

$$
v_{n}:=u_{n} * \tilde{h}_{0, n}+\tilde{k}_{0, n} * w_{n}, \quad n \in \mathbb{N}_{0},
$$

where

$$
\tilde{h}_{0, n}=\mathcal{Z}^{-1}\left\{\hat{\tilde{h}}_{0}(z)\right\}, \quad \tilde{k}_{0, n}=\mathcal{Z}^{-1}\left\{\hat{\tilde{k}}_{0}(z)\right\} .
$$

Using Plancherel's Theorem for $Z$-transforms we can now show that

$$
\begin{aligned}
\Delta t & \operatorname{Im} \sum_{n=0}^{N}\left(\bar{\eta}_{0, n}+\beta \bar{\eta}_{0, n+1}\right) \Delta^{+}\left(\eta_{0, n}+\beta \eta_{0, n+1}\right) \\
& =\Delta t \operatorname{Im} \sum_{n=0}^{N}\left(\bar{\eta}_{1, n}+\beta \bar{\eta}_{1, n+1}\right) \Delta^{-}\left(\eta_{1, n}+\beta \eta_{1, n+1}\right)=\Delta t \operatorname{Im} \sum_{n=0}^{\infty} \bar{u}_{n} v_{n} \\
& =\frac{\Delta t}{2 \pi} \int_{0}^{2 \pi}\left|\hat{u}\left(e^{i \varphi}\right)\right|^{2} \operatorname{Im} \hat{\tilde{h}}_{0}\left(e^{i \varphi}\right)+\operatorname{Im}\left\{\hat{\tilde{k}}_{0}\left(e^{i \varphi}\right) \hat{w}\left(\beta e^{i \varphi}\right) \overline{\hat{u}}\left(e^{i \varphi}\right)\right\} d \varphi \\
& \leq(1+\beta)^{2}\left\|\hat{\tilde{k}}_{0}\left(e^{i \varphi}\right)\right\|_{L^{\infty}(0,2 \pi)}\left\|\beta^{-n} \psi_{1}\right\|_{\ell^{2}(0, N)}\left\|\eta_{1}\right\|_{\ell^{2}(0, N)},
\end{aligned}
$$

since $\operatorname{Im} \hat{\tilde{h}}_{0}\left(e^{i \varphi}\right) \leq 0$ for all $\beta \geq 1$. An analogous estimate holds for the right boundary term. The discrete $L^{2}$-norm in (6.34) is defined by $\left\|\eta_{j}\right\|_{\ell^{2}(0, N)}=\Delta t \sum_{n=0}^{N}\left|\eta_{j, n}\right|^{2}$. For estimating the boundary term $\left\|\eta_{1}\right\|_{\ell^{2}(0, N)}$ we use (6.25):

$$
\begin{aligned}
\left|\eta_{1, n}\right|^{2} & =\beta^{-2 n}\left|\mu_{1, n}-\psi_{1, n}\right|^{2} \\
& \leq 2\left\{\left(\frac{\beta_{1}}{\beta}\right)^{2 n}\left\|\psi_{0}\right\|_{2}^{2}+\left(\frac{c}{\beta}\right)^{2 n} X\left[\left\|\Delta^{+} \psi_{0}\right\|_{2}^{2}+\frac{\left(1+\beta_{1}\right)^{2}}{2 \beta_{2}^{2}} \frac{\left\|\psi_{0}\right\|_{2}^{2}}{2-\Delta t} V_{\Delta^{+}, n-1}^{\max }\right]\right\}
\end{aligned}
$$

$n=0, \ldots, N+1$. Summing for $n=0, \ldots, N$ yields

$$
\begin{aligned}
\left\|\eta_{1}\right\|_{\ell^{2}(0, N)}^{2} \leq 2 \Delta t & \left\{\frac{1-\left(\frac{\beta_{1}}{\beta}\right)^{2(N+1)}}{1-\frac{\beta_{1}^{2}}{\beta^{2}}}\left\|\psi_{0}\right\|_{2}^{2}+\frac{1-\left(\frac{c}{\beta}\right)^{2(N+1)}}{1-\frac{c^{2}}{\beta^{2}}} X\left\|\Delta^{+} \psi_{0}\right\|_{2}^{2}\right. \\
& \left.+X \frac{\left(1+\beta_{1}\right)^{2}}{2 \beta_{2}^{2}} \frac{\left\|\psi_{0}\right\|_{2}^{2}}{2-\Delta t} \sum_{n=0}^{N}\left(\frac{c}{\beta}\right)^{2 n} V_{\Delta^{+}, n-1}^{\max }\right\}
\end{aligned}
$$

and an analogous estimate holds for $\left\|\beta^{-n} \psi_{1}\right\|_{\ell^{2}(0, N)}$, i.e. we obtain (6.28).

REMARK 8. Instead of the discrete Sobolev-inequality (6.25) one could also use the following trivial $L^{2}$-estimates in (6.36):

$$
\left|\eta_{1, n}\right| \leq \frac{1}{\sqrt{\Delta x}}\left\|\eta_{n}\right\|_{2}, \quad\left|\psi_{1, n}\right| \leq \frac{1}{\sqrt{\Delta x}}\left\|\psi_{n}\right\|_{2}
$$


This would imply

$$
\left\|\beta^{-n} \psi_{1}\right\|_{\ell^{2}(0, N)}\left\|\eta_{1}\right\|_{\ell^{2}(0, N)} \leq 2 \frac{\Delta t}{\Delta x}\left\|\psi_{0}\right\|_{2}^{2} \sum_{n=0}^{N} \beta^{-2 n}\left(1+\beta_{1}^{2 n}\right)
$$

i.e.

$$
\left\|\eta_{N+1}\right\|_{2}^{2} \leq \frac{(1+\beta)^{2}}{2 \beta^{2}} \frac{\Delta t}{\Delta x} g_{0, J}^{\max }\left\|\psi_{0}\right\|_{2}^{2} \sum_{n=0}^{N} \beta^{-2 n}\left(1+\beta_{1}^{2 n}\right) .
$$

This yields finally the error estimate

$$
\left\|\kappa_{n}\right\|_{2}^{2} \leq g_{0, J}^{\max } \frac{\left\|\psi_{0}\right\|_{2}^{2}}{2 \Delta x} \cdot \Delta t(1+\beta)^{2}\left[\frac{\beta^{2 n}-1}{\beta^{2}-1}+\frac{\beta^{2 n} \beta_{1}^{2 n}}{\beta^{2}-\beta_{1}^{2}}\right] .
$$

This estimate can be applied if we assume that $\Delta x=$ const (which corresponds to Case 2 of Section 5, cf. Theorem 5.1). The advantage of (6.38) is that the condition (6.14) is not necessary.

Note that $g_{0, J}^{\max }$ is the discrete analogue of $f_{0, X}^{\max }$ from (6.10). With the standard connection between Laplace- and $Z$-transforms we have:

$$
\frac{\hat{g}_{0}^{\mathrm{TBC}}\left(e^{s \Delta t}\right)-1}{\Delta x} \rightarrow \hat{f}_{0}^{\mathrm{TBC}}(s), \quad \Delta x, \Delta t \rightarrow 0,
$$

$s=\alpha+i \xi$ fixed.

Example 6.5. ("cut-off" DTBC) We consider the simplified DTBC of Example 5.4 where the convolution coefficients $s_{j, n}$ are cut off for $n \geq N$. In Figure 6.1 we verify for $N=10$ that the corresponding transformed convolution kernel (cf. (5.25)) satisfies the condition for the $h^{1}$-a-priori estimate (6.14a) only for $\beta_{2} \geq 1.24$.

As a discrete analogue of Example 6.2 we shall now illustrate the error estimate of Theorem 6.2: In (6.28) the error between the two solutions is bounded by

$$
\left\|\frac{1}{\hat{\ell}_{j}^{(N)}\left(\beta e^{i \varphi}\right)}-\frac{1}{\hat{\ell}_{j}\left(\beta e^{i \varphi}\right)}\right\|_{L^{\infty}(0,2 \pi)}, j=0, J,
$$

the difference between the exact $\left(\left\{\ell_{j, n}\right\}\right)$ and the "cut-off" convolution kernels $\left(\left\{\ell_{j, n}^{(N)}\right\}\right)$.

In the following table we show, how this difference (6.39) decreases as $N$, the number of retained convolution coefficients, grows. Theorem 6.2 hence implies convergence (as $N \rightarrow \infty$ ) of the corresponding discrete Schrödinger solutions for the scheme (5.10). The parameters are again $\Delta x=1 / 160, \Delta t=2 \cdot 10^{-5}, V \equiv 0$, and $\beta=1.25$.

\begin{tabular}{|c|r|r|r|r|r|}
\hline$N=$ & 5 & 10 & 20 & 30 & 40 \\
\hline error $\left\|1 / \hat{\ell}_{0}^{(N)}-1 / \hat{\ell}_{0}\right\|_{\infty}$ & 1.7346 & 0.97592 & 0.04391 & 0.004559 & 0.00048768 \\
\hline
\end{tabular}

Example 6.6. (approximated DTBC) We shall now compare the above error estimate (6.28), (6.39) to the situation in the approximated DTBC with the sumof-exponentials-ansatz (3.1) (with $\nu=2$ ). In the following table we show, how the difference (6.39) decreases as $L$ grows. Note that the numerical effort for evaluation 


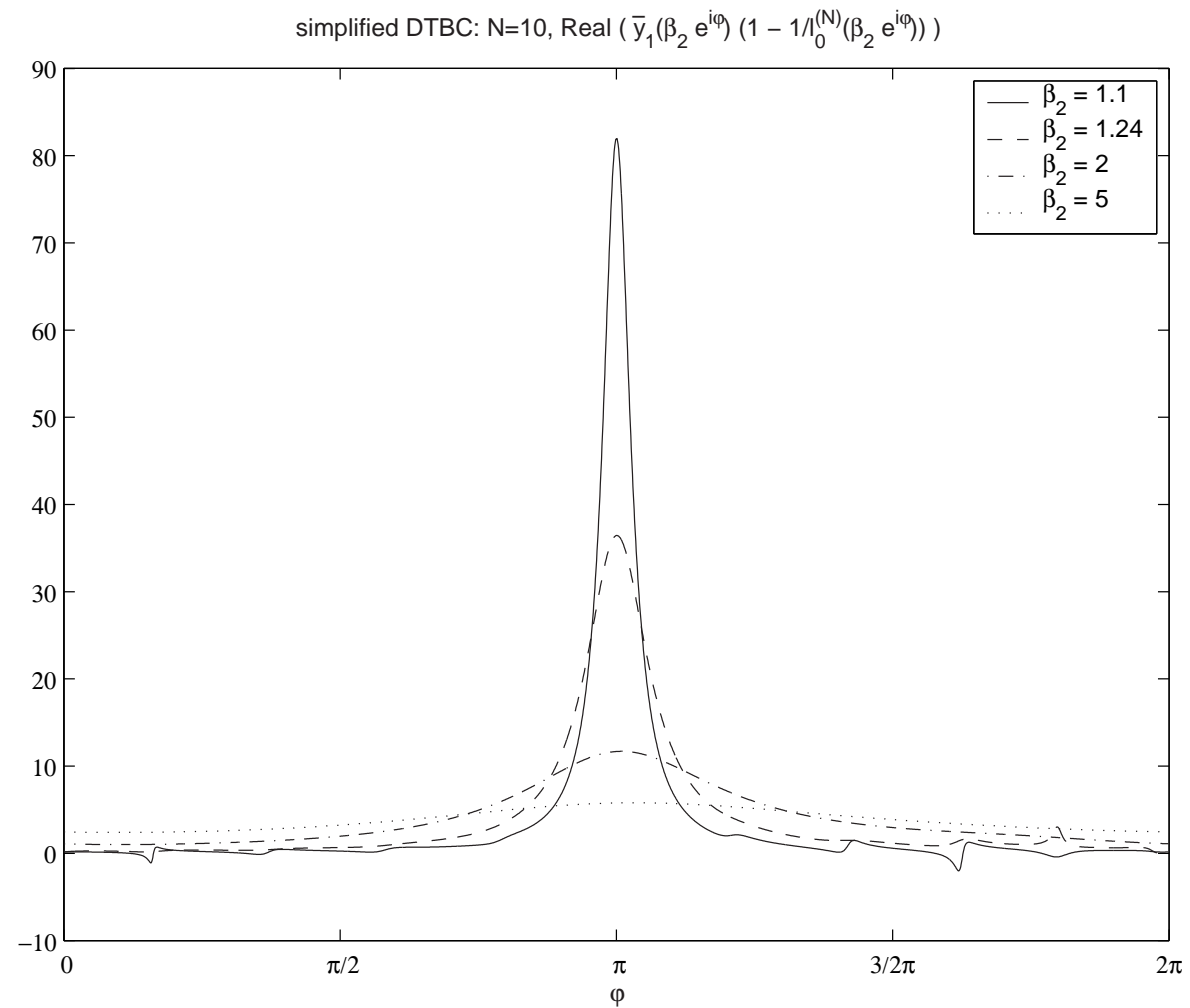

FIG. 6.1. $h^{1}$-condition (6.14a) for simplified discrete transparent boundary conditions with cutoff after $N=10$ convolution coefficients, $\Delta x=1 / 160, \Delta t=2 \cdot 10^{-5}, V \equiv 0$. The $h^{1}$-condition (6.14a) is satisfied for $\beta_{2} \geq 1.24$.

the DTBC is the same in both cases when $2 L=N$ (compared to Example 6.5). On the other hand, the error bounds are much smaller for the sum-of-exponentials-ansatz. Hence, the corresponding solution is much more accurate (cf. Example 7.1, below).

\begin{tabular}{|c|r|r|r|r|r|}
\hline$L=$ & 3 & 15 & 10 & 15 & 20 \\
\hline error $\left\|1 / \hat{\tilde{\ell}}_{0}-1 / \hat{\ell}_{0}\right\|_{\infty}$ & 0.0029642 & 0.00018247 & $1.2808 \mathrm{e}-07$ & $6.4439 \mathrm{e}-11$ & $2.962 \mathrm{e}-14$ \\
\hline
\end{tabular}

\section{Numerical examples}

In this section we shall present two examples to compare the numerical results of using our new approach of the approximated DTBC with the sum-of-exponentialsansatz (3.1) (with $\nu=2$ ) with the solution using the exact DTBC (1.17).

Example 7.1. As a first example we consider the Schrödinger equation (1.1) in one space dimension on $0 \leq x \leq 1$ with $V \equiv 0$, and initial data $\psi^{I}(x)=\exp (i 100 x-$ $\left.30(x-0.5)^{2}\right)$. The time evolution of the approximate solution $\left|\psi_{a}(x, t)\right|$ using the approximated DTBC with convolution coefficients $\left\{\tilde{s}_{n}\right\}$ and $L=10, L=20$ is shown, respectively, in Figure 7.1 and Figure 7.2 (observe the viewing angle).

While one can observe some reflected wave when using the approximated DTBC with $L=10$, there are no reflections visible when using the approximated DTBC with 


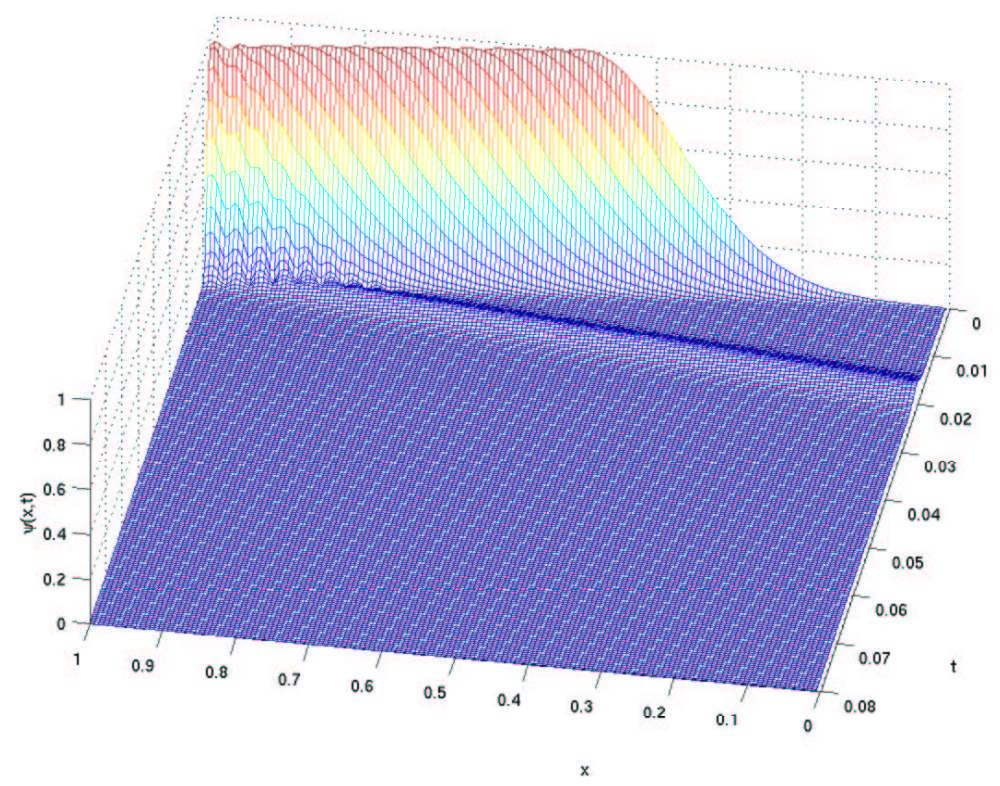

FIG. 7.1. Time evolution of $\left|\psi_{a}(x, t)\right|$ : The approximate convolution coefficients consisting of $L=10$ discrete exponentials give rise to a reflected wave.

$L=20$.

Next we investigate the long-time stability behaviour of the approximated DTBC with the sum-of-exponentials ansatz. The reference solution $\psi_{r e f}$ with $\Delta x=1 / 160$, $\Delta t=2 \cdot 10^{-5}$ is obtained by using exact DTBCs (1.17) at the end points $x=0$ and $x=1$. We vary the parameter $L=20,30,40,50$ in (3.1) to find the corresponding approximate DTBCs, and show the error of the approximate solution $\psi_{a}$ measured in $\left\|\psi_{a}(t)-\psi_{\text {ref }}(t)\right\|_{L_{2}} /\left\|\psi^{I}\right\|_{L_{2}}$. The result up to time step $n=15000$ is shown in the Figure 7.3(a). Larger values of $L$ clearly yield more accurate coefficients and hence a more accurate solution $\psi_{a}$.

In Figure 7.3(b) we show the analogous result for the "cut-off" DTBC, where we retained $N=20,30,40,50$ exact convolution coefficients. While the numerical effort is the same for both approaches with $2 L=N$, our sum-of-exponentials DTBC yields an error that is 3-4 orders of magnitude smaller.

Example 7.2. The second example considers the time evolution of a wave function in a potential well of finite depth: We solve the 1D-Schrödinger equation (1.1) on $[0,2]$ with zero potential in the interior $(V(x) \equiv 0$ for $0<x<2)$ and $V(x) \equiv 4500$ outside the computational domain. Figure 7.4 shows the time evolution of a right travelling Gaussian beam $\left[\psi^{I}(x)=\exp \left(i 100 x-30(x-1)^{2}\right)\right]$ using the rather coarse space discretization $\Delta x=1 / 160$, the time step $\Delta t=2 \cdot 10^{-5}$, and the exact DTBC (1.17). We observe in Figure 7.4 that the main part of the wave is reflected at the boundaries. The value of the potential is chosen such that at time $t=0.08$, i.e. after 4000 time steps $75 \%$ of the mass $\left(\|\psi(., t)\|_{2}^{2}\right)$ has left the domain.

While the discrete TBCs (1.17) yield the exact numerical solution to the discrete whole-space problem (up to round-off errors), the approximated DTBC induces small 


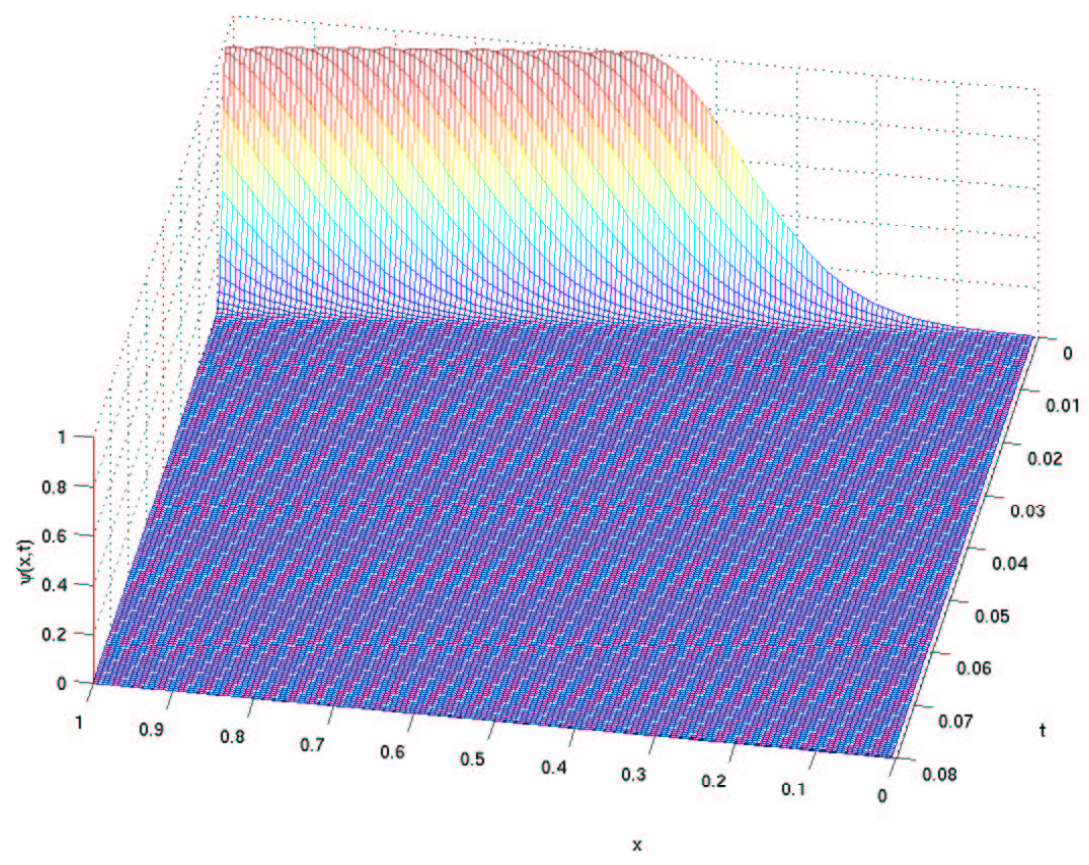

FIG. 7.2. Time evolution of $\left|\psi_{a}(x, t)\right|$ : The approximate convolution coefficients consisting of $L=20$ discrete exponentials make reflections already invisible.

errors. Figure 7.5 shows the error of the approximate solution $\psi_{a}$ defined by $e_{L}(x, t):=$ $\left(\psi_{a}(x, t)-\psi_{\text {ref }}(x, t)\right) /\left\|\psi^{I}\right\|_{L_{2}}$.

Figure 7.6 shows the time decay of the discrete $\ell^{2}$-norm $\|\psi(., t)\|_{2}$ and the temporal evolution of the error $\left\|e_{L}(., t)\right\|_{2}$ when using an approximated DTBC with $L=20,30,40$. Additionally, we calculated for $L=20$ the coefficients $\left\{b_{l}, q_{l}\right\}$ for the "normalized parameters" $\Delta x=1, \Delta t=1, V=0$ (cf. Appendix A) and then used the Transformation rule 3.1 to calculate the coefficients $\left\{b_{l}^{*}, q_{l}^{*}\right\}$ for the desired parameters (cf. Example 3.1). The result is better than calculating the convolution coefficients "directly" (compare the error-curves " $L=20$ (trafo)" and " $L=20$ "). One observes that the error increases with time. This is not surprising since each reflection at the boundaries induces an additional error.

Evaluating the convolution appearing in exact DTBCs is quite expensive for longtime calculations. Therefore we shall now illustrate the difference in the computational effort for both approaches in Figure 7.7:

The computational effort for the exact DTBCs is quadratic in time, since the evaluation of the boundary convolutions dominates for large times. On the other hand, the effort for the approximated DTBC only increases linearly. For $L=10,20,30$ the lines are indistinguishable since the evaluation of the sum-of-exponential convolutions has a negligible effort compared to solving the PDE in the interior domain. 

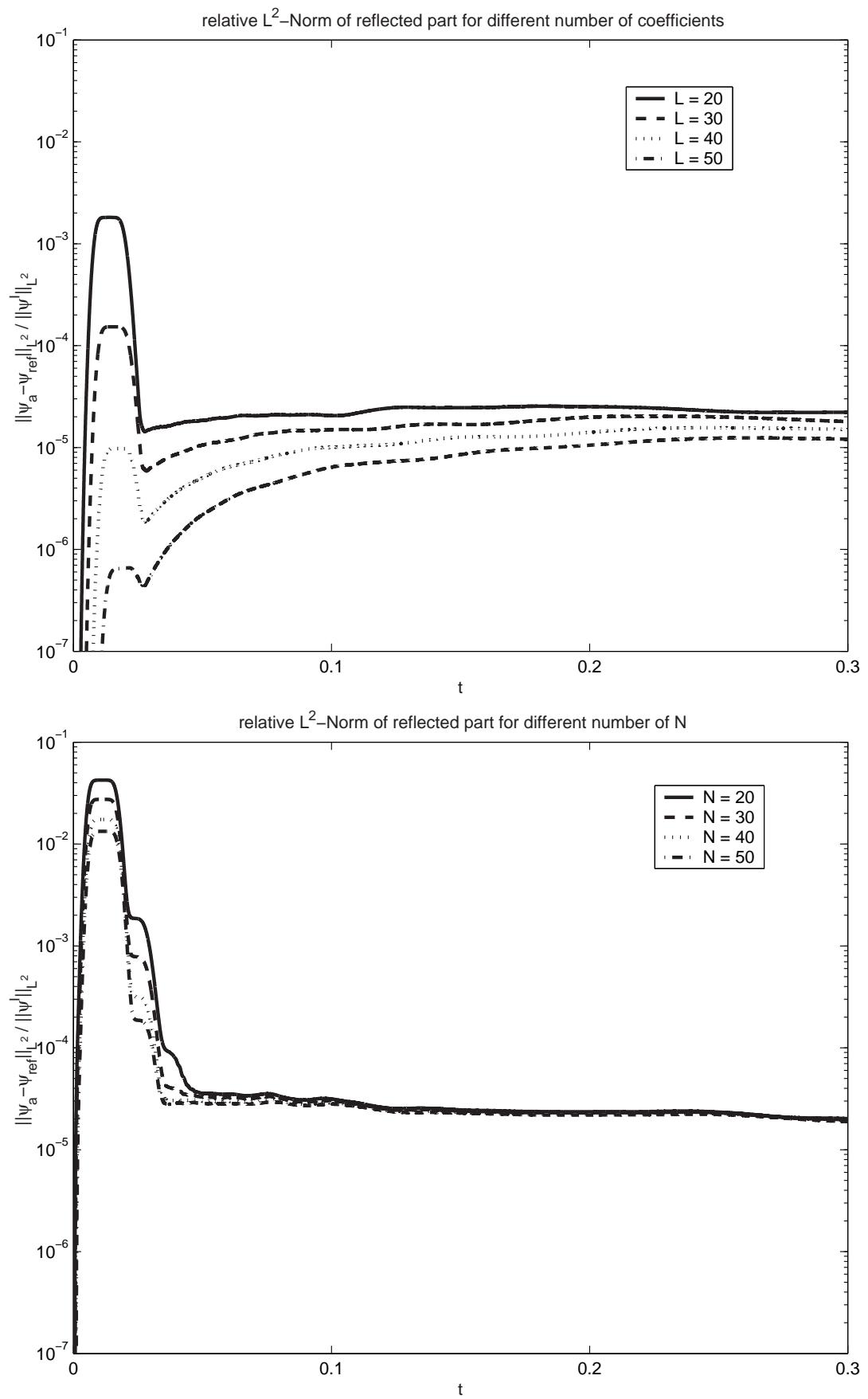

FIG. 7.3. Error of the approximate solution $\psi_{a}(t)$ with (a) approximate convolution coefficients consisting of $L=20,30,40,50$ discrete exponentials; (b) "cut-off" DTBC: The convolution coefficients are cut off for $n \geq N$ with $N=20,30,40,50$. The error-peak between $t=0.01$ and $t=0.02$ corresponds to the first reflected wave, which is clearly visible in Figure 7.1. 


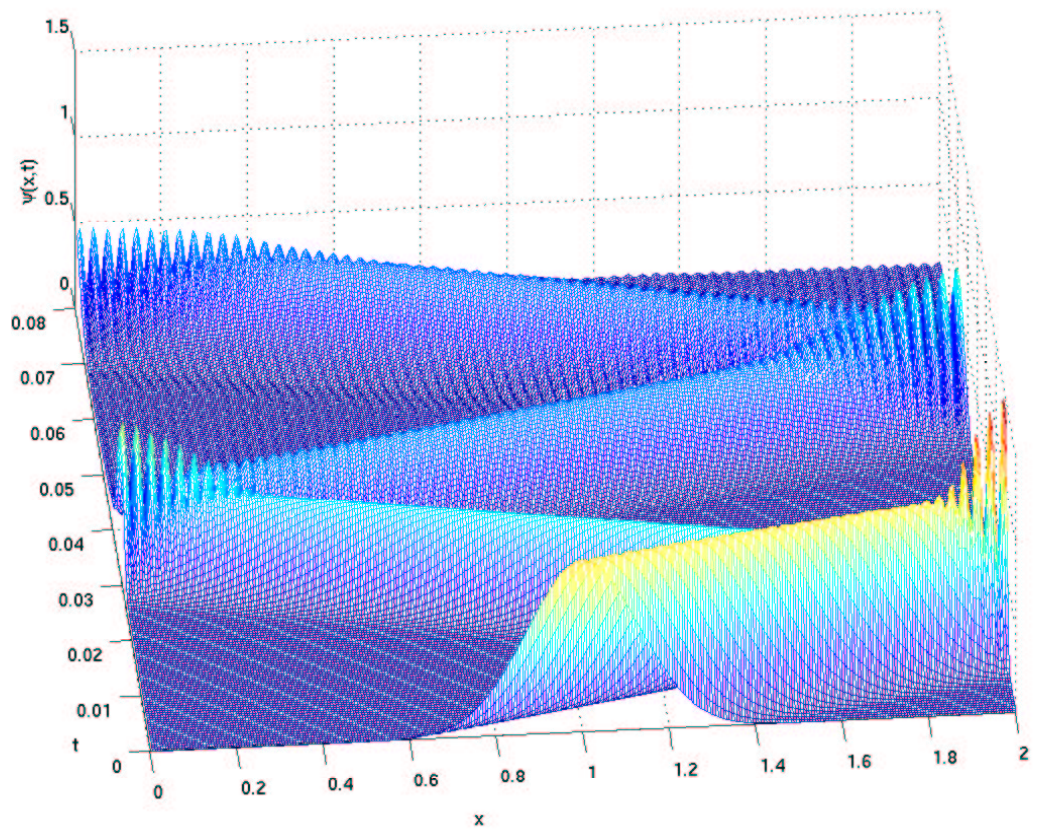

FIG. 7.4. Time evolution of $|\psi(x, t)|$ in a potential well. The Gaussian beam is almost perfectly reflected by the walls of height $V=4500$.

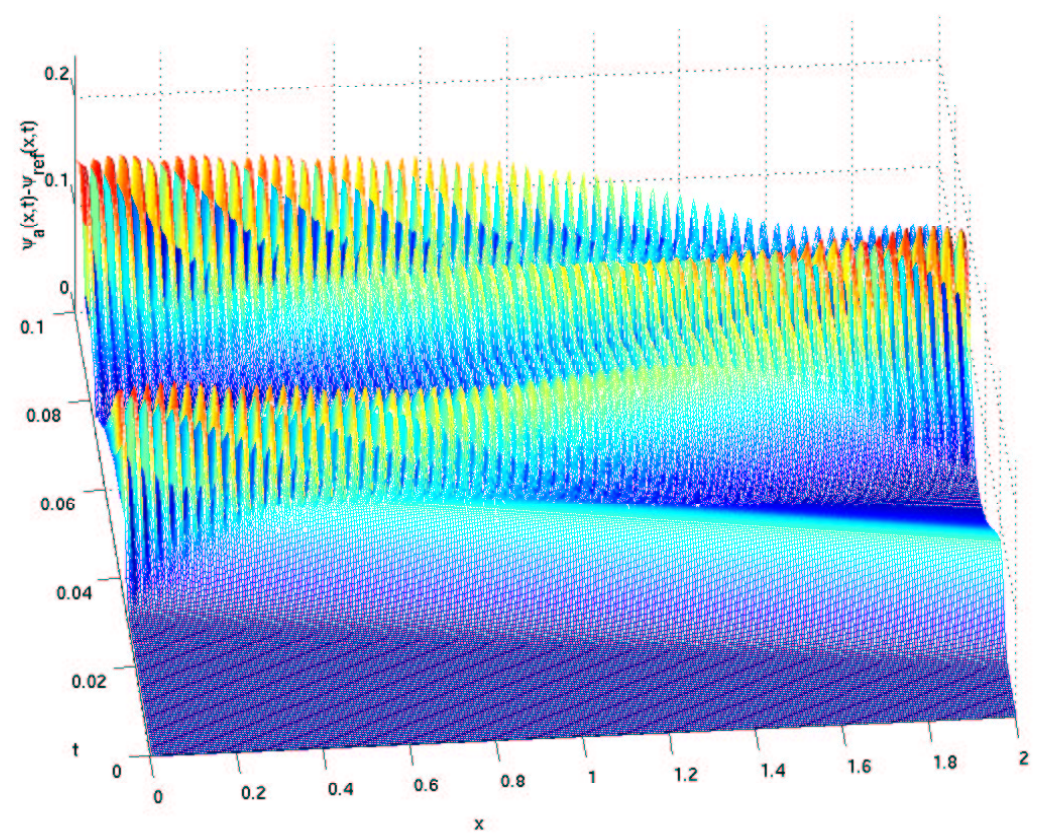

FIG. 7.5. Time evolution within the potential well $(V=4500)$ of the error $\left|e_{L}(x, t)\right|$ due to an approximated $D T B C$ with $L=20$. As expected, the error accumulates with each reflection of the main wave. 


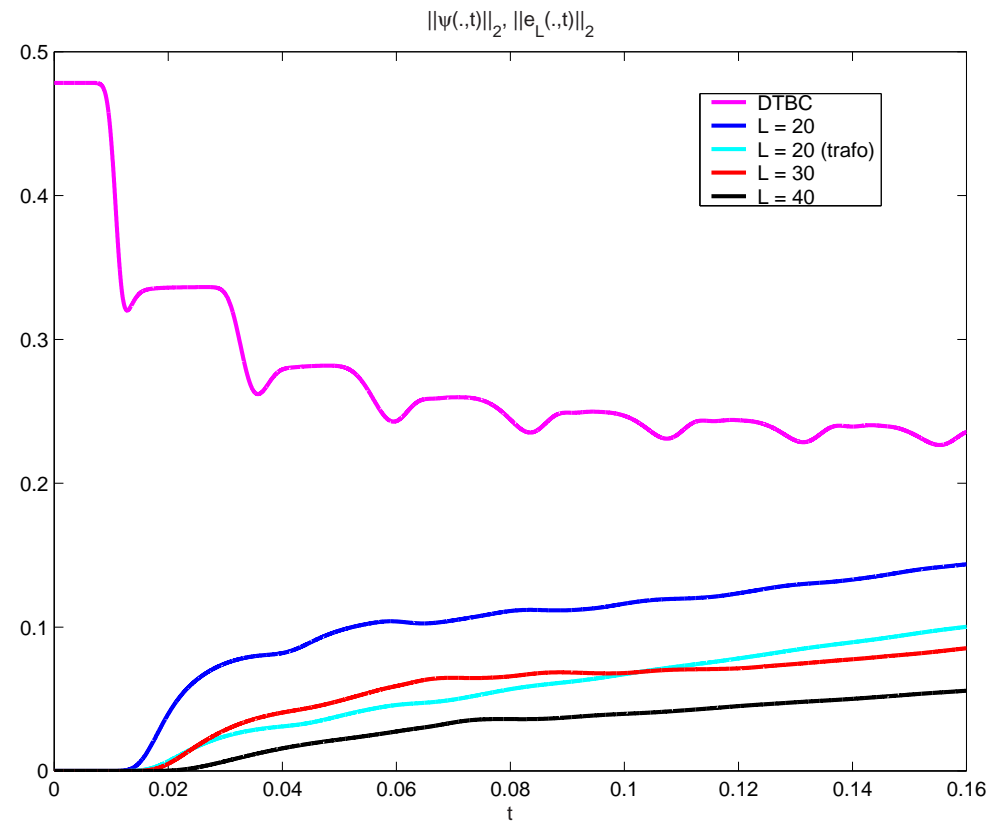

FIG. 7.6. Time evolution within the potential well $(V=4500)$ of the discrete $\ell^{2}$-norm $\|\psi(., t)\|_{2}$ and of the errors $\left\|e_{L}(., t)\right\|_{2}$ that are due to approximated DTBCs with $L=20,30,40$. " $L=20$ (trafo)" uses convolution coefficients calculated by the Transformation 3.1.

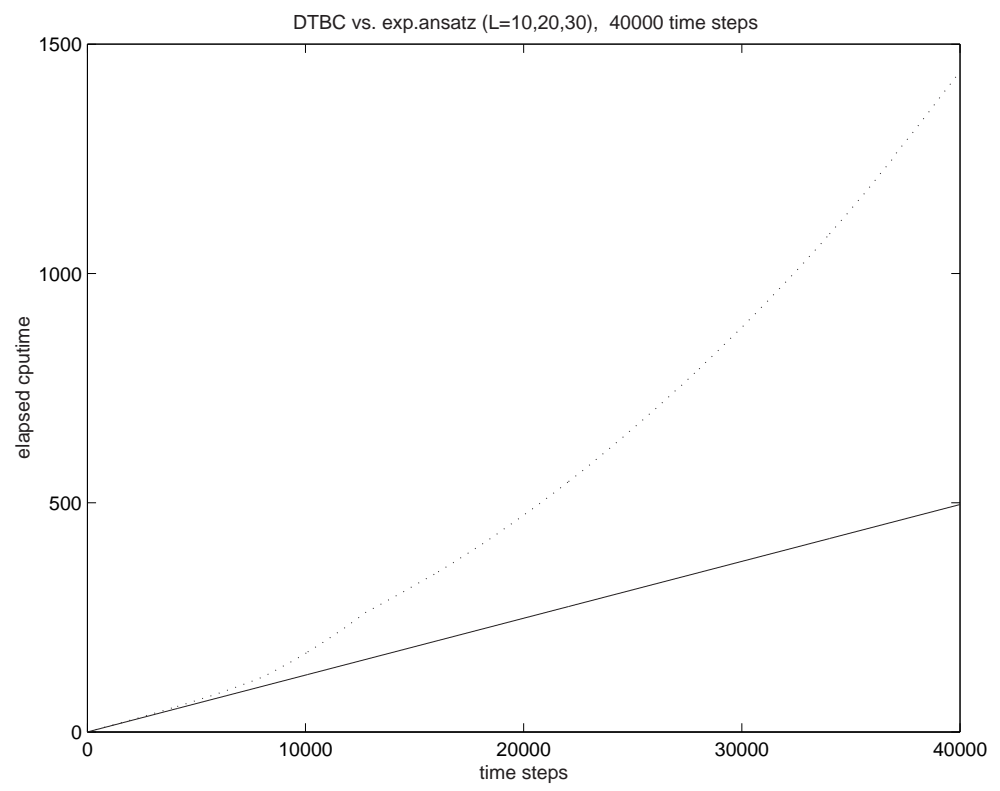

FIG. 7.7. Comparison of CPU times: the sum-of-exponential approximation to DTBCs has linear effort (-), while exact DTBCs have quadratic effort. 
Appendix A. In the following table we list the coefficients $\left\{q_{l}, b_{l}\right\}$ of the exponentialsums-BC having the convolution kernel (3.1) for the cases $L=5$, and $L=10$ with the "normalized parameters" $\nu=2, \Delta x=\Delta t=1$, and $V=0$ :

\begin{tabular}{|c|c|c|}
\hline & $q_{l}$ & $b_{l}$ \\
\hline \multirow{5}{*}{$\mathrm{L}=5$} & $1.0613253+0.83506991 \mathrm{e}-1 * \mathrm{I}$ & $-0.46123493 \mathrm{e}-1-0.35384269 \mathrm{e}-1 * \mathrm{I}$ \\
\hline & $0.83506991 \mathrm{e}-1+1.0613253 * \mathrm{I}$ & $0.57634691 \mathrm{e}-1+0.75937784 \mathrm{e}-2 * \mathrm{I}$ \\
\hline & $1.1653982+0.41107342^{*} \mathrm{I}$ & $-0.95195640 \mathrm{e}-1-0.20683503^{*} \mathrm{I}$ \\
\hline & $0.41107342+1.1653982 * \mathrm{I}$ & $0.21356793-0.78940966 \mathrm{e}-1 * \mathrm{I}$ \\
\hline & $0.95734921+0.95734921 * \mathrm{I}$ & $0.13684972-0.33038444 * \mathrm{I}$ \\
\hline \multirow{10}{*}{$\mathrm{L}=10$} & $1.0204790+0.20818849 \mathrm{e}-1 * \mathrm{I}$ & $-0.81657939 \mathrm{e}-2-0.36037147 \mathrm{e}-2 * \mathrm{I}$ \\
\hline & $0.20818849 \mathrm{e}-1+1.0204790 * \mathrm{I}$ & $0.83222994 \mathrm{e}-2+0.32258771 \mathrm{e}-2 * \mathrm{I}$ \\
\hline & $1.0793585+0.91985074 \mathrm{e}-1 * \mathrm{I}$ & $-0.31636868 \mathrm{e}-1-0.19266238 \mathrm{e}-1 * \mathrm{I}$ \\
\hline & $0.91985074 \mathrm{e}-1+1.0793585^{*} \mathrm{I}$ & $0.35993931 \mathrm{e}-1+0.87473565 \mathrm{e}-2 * \mathrm{I}$ \\
\hline & $1.1613828+0.24003468 * \mathrm{I}$ & $-0.61222441 \mathrm{e}-1-0.61543495 \mathrm{e}-1 * \mathrm{I}$ \\
\hline & $0.24003468+1.1613828 * \mathrm{I}$ & $0.86808626 \mathrm{e}-1-0.22701935 \mathrm{e}-3^{*} \mathrm{I}$ \\
\hline & $1.2133719+0.49825873 * \mathrm{I}$ & $-0.60889615 \mathrm{e}-1-0.14138908^{*} \mathrm{I}$ \\
\hline & $0.49825873+1.2133719 * \mathrm{I}$ & $0.14303264-0.56921716 \mathrm{e}-1 * \mathrm{I}$ \\
\hline & $1.1272018+0.84466563 * \mathrm{I}$ & $0.22117261 \mathrm{e}-1-0.20911201 * \mathrm{I}$ \\
\hline & $0.84466563+1.1272018 * \mathrm{I}$ & $0.13222525-0.16350378 * \mathrm{I}$ \\
\hline
\end{tabular}

The coefficients $b_{l}^{*}, q_{l}^{*}$ for other parameters $\Delta x_{*}, \Delta t_{*}, V_{*}$ can then be obtained from the explicit formulas in the Transformation rule 3.1. A Java-Applet for calculating $b_{l}^{\star}, q_{l}^{\star}$ is available on the authors' homepages: www.math.uni-muenster.de/u/arnold/dtbc.html, www.math.tu-berlin.de/ ${ }^{\text {ehrhardt/ }}$

Appendix B. Here we present the Maple code that was used to calculate the coefficients $q_{l}, b_{l}$ in the approximation (3.1) including the explicit formulae in Transformation rule 3.1. These codes can also be downloaded from the authors' homepages. We note that the Padé approximation must be performed with high precision $(2 L-1$ digits mantissa length) to avoid a 'nearly breakdown' by ill conditioned steps in the Lanczos algorithm (cf. [14]). If such problems still occur or if one root of the denominator is smaller than 1 in absolute value, the orders of the numerator and denominator polynomials are successively reduced ('cycle A').

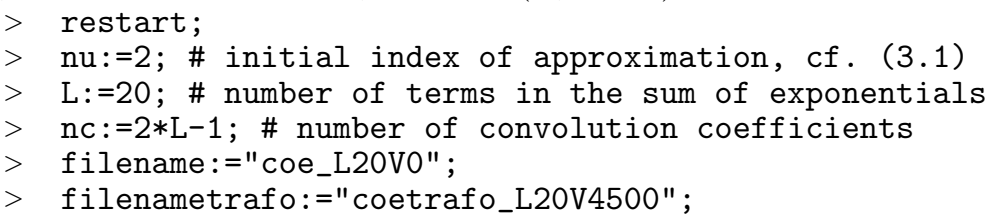




\section{Numerical inverse Z-transformation of the given analytical kernel (hat_s).}

The function 'hat_s', see (1.11), (1.13), is considered outside the unit circle.

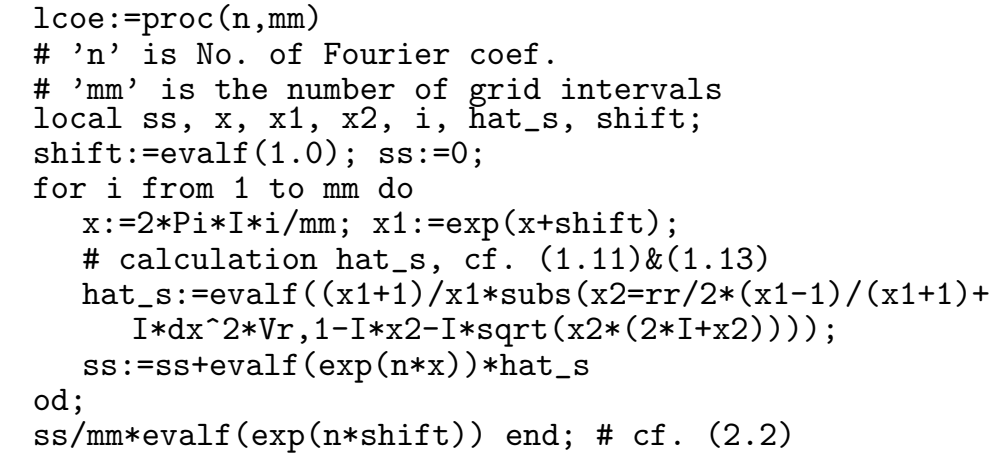

Calculation of the convolution coefficients:

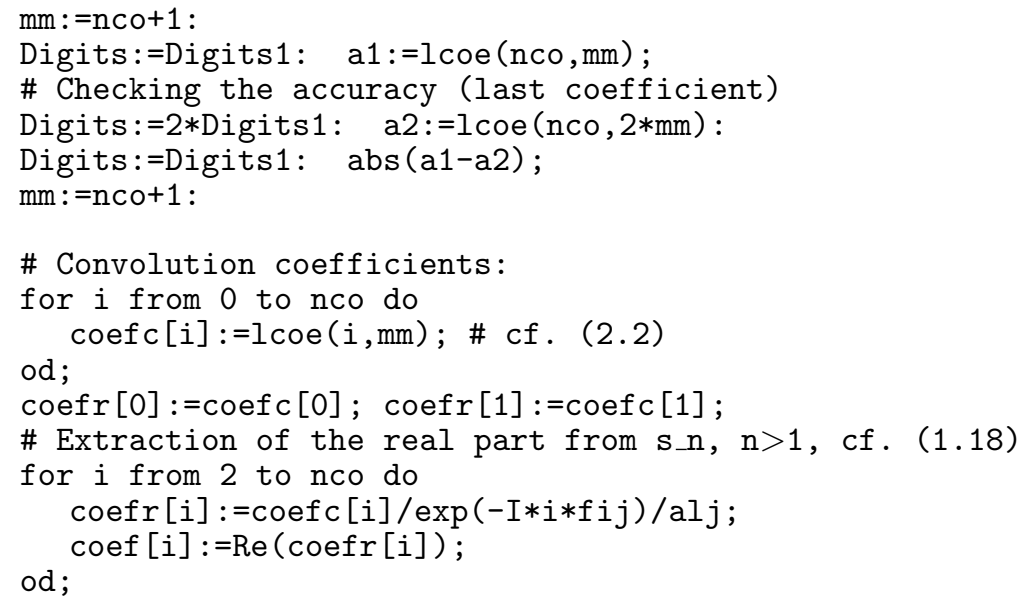

3. Approximation.

Calculation of the polynomial 'sp'. Only the coefficients starting with $\mathrm{n}=\mathrm{nu}$ are used here; i.e. coef[0], coef[1], ., coef[nu-1] are not considered.

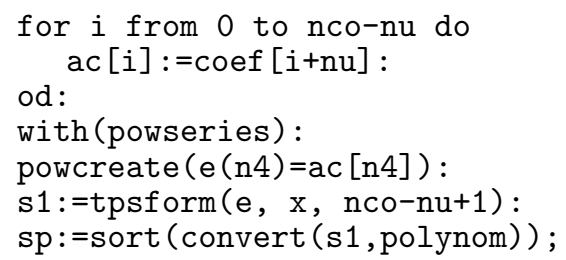

Calculation of a rational function approximating the polynomial 'sp'. This is the usual Padé algorithm. The parameter 'npow' defines the orders of the numerator and denominator. Important: We have to check that the roots of the denominator are larger than 1 in absolute value. The value of 'npow' influences this property: Cycle A automatically chooses smaller and smaller values of 'npow' (L-1, L-2,..) to guarantee that all roots have an absolute value larger than 1. 


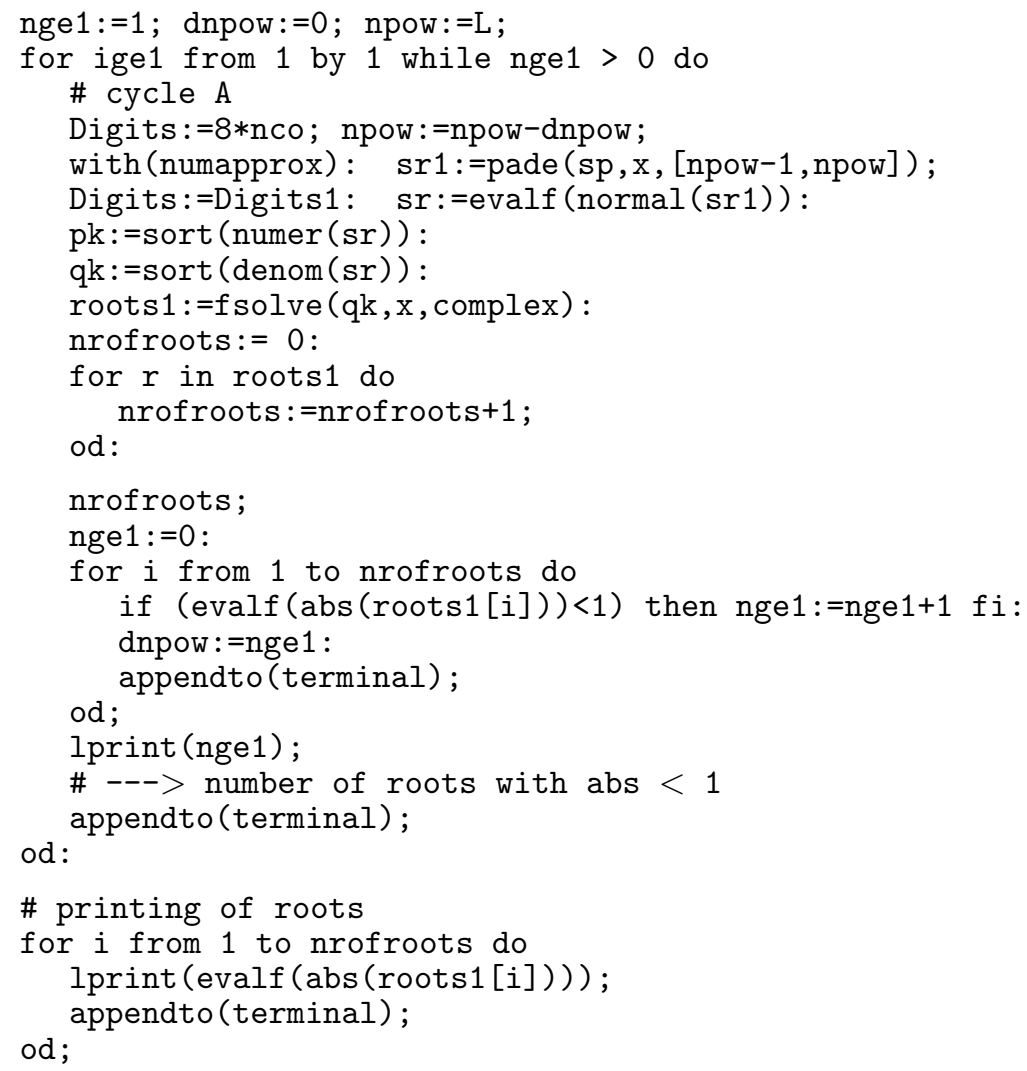

Writing of the result:

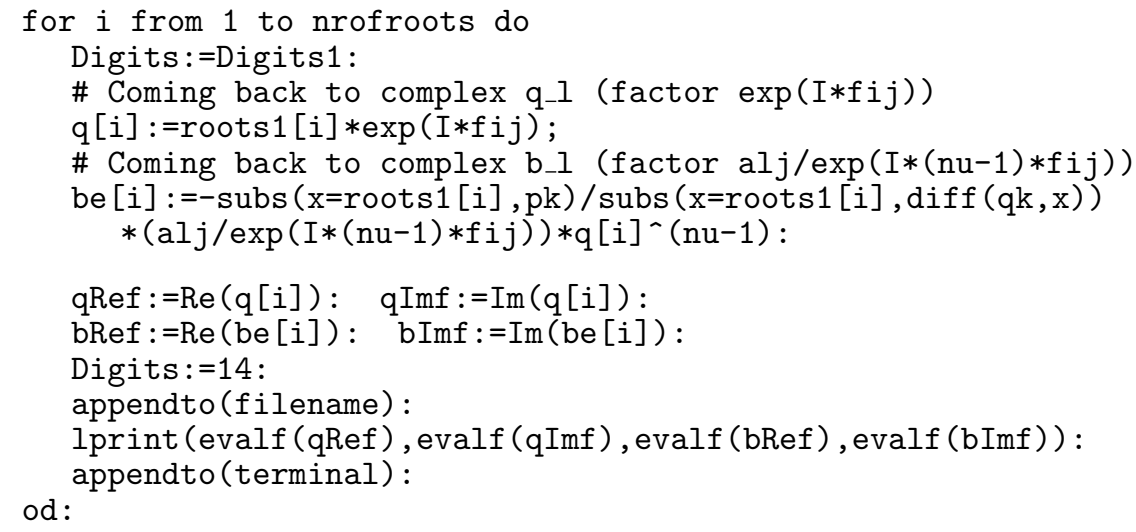

Checking of our representation:

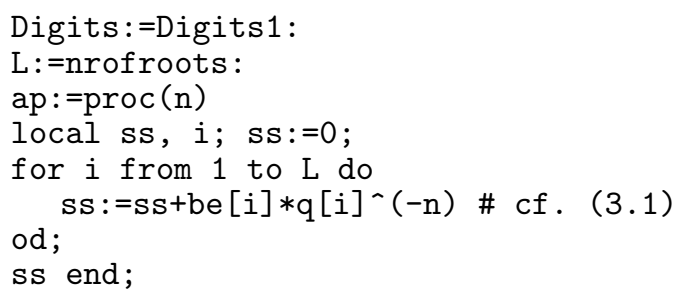

First nco coefficients: 


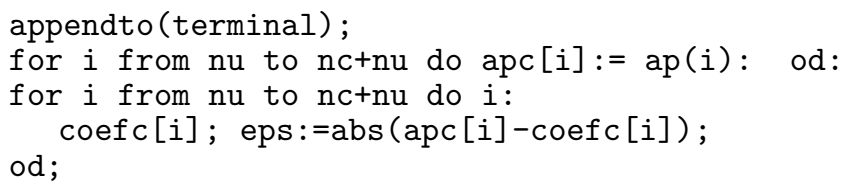

4. Transformation to other grid parameters, see Transformation 3.1.

First nco new convolution coefficients (starting with $\mathrm{n}=\mathrm{nu}$ ):

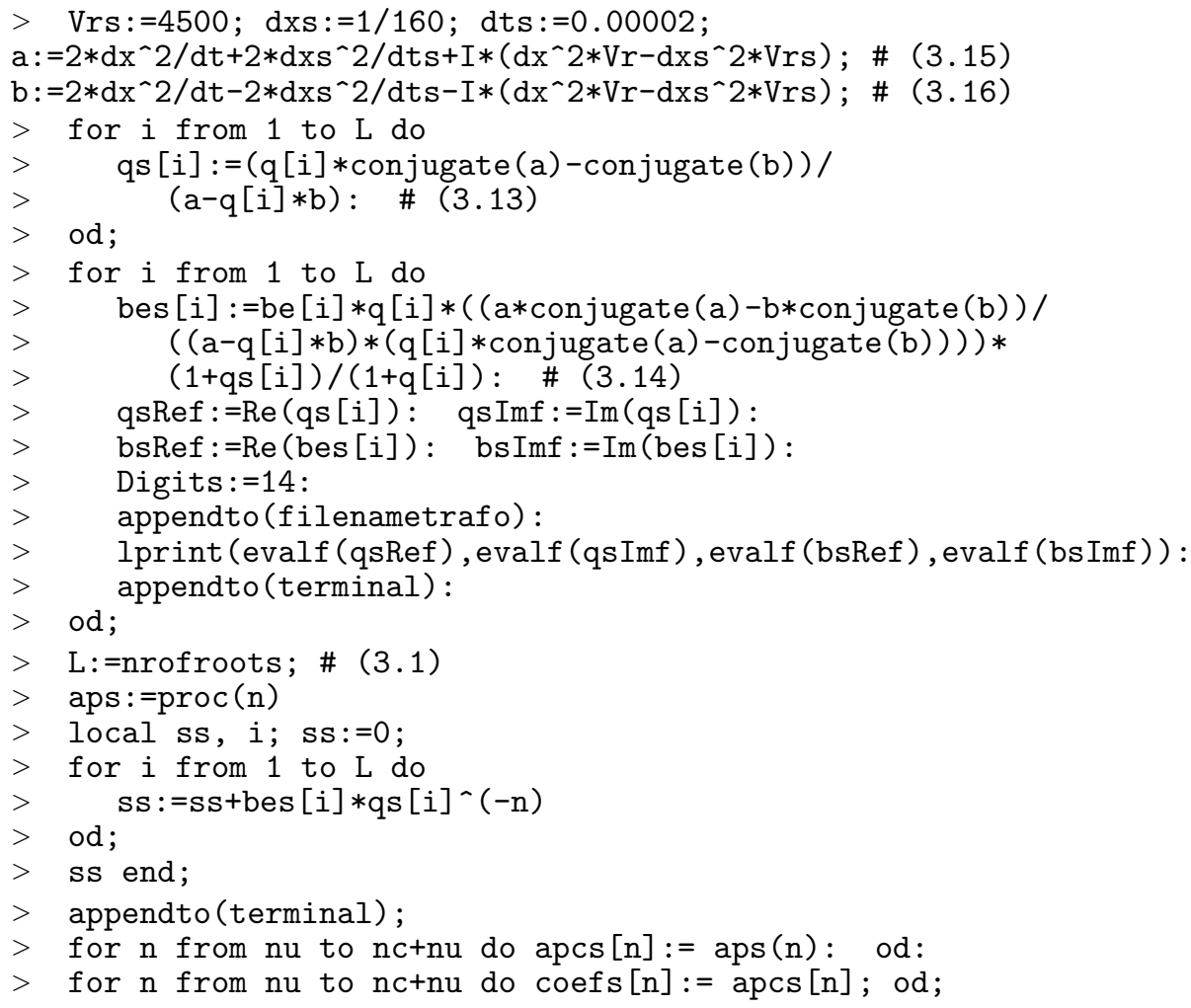

Acknowledgement. The first two authors were partially supported by the grants ERBFMRXCT970157 (TMR-Network) from the EU and the DFG under GrantNo. AR 277/3-1. The second author was supported by the DFG Research Center "Mathematics for key technologies" (FZT 86) in Berlin. The third author was partially supported by RFBR-Grant No. 01-01-00520 and by Saarland University.

The authors acknowledge very stimulating discussions with Christian Lubich and Achim Schädle.

The authors are grateful to the numerous suggestions made by the anonymous referees that strongly helped to clarify many aspects of this paper.

\section{REFERENCES}

[1] B. Alpert, L. Greengard, and T. Hagstrom, Rapid evaluation of nonreflecting boundary kernels for time-domain wave propagation, SIAM J. Numer. Anal., 37:1138-1164, 2000.

[2] B. Alpert, L. Greengard, and T. Hagstrom, Nonreflecting Boundary Conditions for the TimeDependent Wave Equation, J. Comput. Phys., 180:270-296, 2002.

[3] I.A. Mallo and N. Reguera, Weak ill-posedness of spatial discretizations of absorbing boundary conditions for Schrödinger-type equations, SIAM J. Numer. Anal., 40:134-158, 2002. 
[4] X. Antoine and C. Besse, Unconditionally stable discretization schemes of non-reflecting boundary conditions for the one-dimensional Schrödinger equation, J. Comp. Phys., 188:157$175,2003$.

[5] A. Arnold, Numerically Absorbing Boundary Conditions for Quantum Evolution Equations, VLSI Design., 6:313-319, 1998.

[6] A. Arnold and M. Ehrhardt, Discrete transparent boundary conditions for wide angle parabolic equations in underwater acoustics, J. Comp. Phys., 145:611-638, 1998.

[7] A. Arnold, Mathematical concepts of open quantum boundary conditions, Transp. Theory Stat. Phys., 30(4-6):561-584, 2001.

[8] G.A. Baker, Jr., Essentials of Padé Approximants, Academic Press NY, San Francisco, London, 1975.

[9] V.A. Baskakov and A.V. Popov, Implementation of transparent boundaries for numerical solution of the Schrödinger equation, Wave Motion., 14:123-128, 1991.

[10] N.B. Abdallah, On a multi-dimensional Schrödinger-Poisson Scattering model for semiconductors, J. Math. Phys., 41(7):2241-2261, 2000.

[11] N.B. Abdallah and O. Pinaud, A mathematical model for the transient evolution of a resonant tunneling diode, C. R. Math. Acad. Sci., Paris, 334:283-288, 2002.

[12] G. Blakiewicz and W. Janke, Recursive Convolution Algorithms for Time-Domain Simulation of Electronic Circuits, Comp. Meth. Sci. Techn., 7:91-109, 2001.

[13] C.H. Bruneau and L.D. Menza, Conditions aux limites transparentes et artificielles pour l'équation de Schrödinger en dimension 1 d'espace, C. R. Acad. Sci. Paris, Ser. I 320:89-94, 1995.

[14] A. Bultheel and M. Van Barel, Linear algebra, rational approximation and orthogonal polynomials, Studies in Computational Mathematics 6, North-Holland, 1997.

[15] L. Burgnies, O. Vanbésien, and D. Lippens, Transient analysis of ballistic transport in stublike quantum waveguides, Appl. Phys. Lett., 71(6):803-805, 1997.

[16] A. Dedner, D. Kröner, I. Sofronov, and M. Wesenberg, Transparent boundary conditions for mhd simulations in stratified atmospheres, J. Comp. Phys., 171:448-478, 2001.

[17] L. Di Menza, Approximations numériques d'équations de Schrödinger non linéaires et de modèles associés, Ph.D. thesis, Université Bordeaux I, 1995.

[18] G. Doetsch, Anleitung zum praktischen Gebrauch der Laplace-Transformation und der ZTransformation, R. Oldenburg Verlag München, Wien, 3. Auflage 1967.

[19] M. Ehrhardt, Discrete transparent boundary conditions for parabolic equations, Z. Angew. Math. Mech., 77(S2):543-544, 1997.

[20] M. Ehrhardt and A. Arnold, Discrete Transparent Boundary Conditions for the Schrödinger Equation, Riv. Mat. Univ. Parma., 6:57-108, 2001.

[21] M.J. Grote and J.B. Keller, Exact nonreflecting boundary conditions for the time dependent wave equation, SIAM J. Appl. Math., 55:280-297, 1995.

[22] T. Hagstrom, Radiation boundary conditions for the numerical simulation of waves, Acta Numerica., 8:47-106, 1999.

[23] J.R. Hellums and W.R. Frensley, Non-Markovian open-system boundary conditions for the time-dependent Schrödinger equation, Phys. Rev. B, 49:2904-2906, 1994.

[24] M.A. Lavrent'ev and B.V. Shabat, Methods for the theory of functions of a complex variable, 5th rev. ed., (Russian), Moskva: Nauka, 1987.

[25] M.F. Levy, Non-local boundary conditions for radiowave propagation, in: Third International Conference on Mathematical and Numerical Aspects of Wave Propagation Phenomena, Juan-les-Pins, France, 24-28 April 1995.

[26] C. Lubich, Convolution quadrature and discretized operational calculus. I, Numer. Math., 52:129-145, 1988.

[27] C. Lubich, Convolution quadrature and discretized operational calculus. II, Numer. Math., 52:413-425, 1988.

[28] C. Lubich, On the multistep time discretization of linear initial-boundary value problems and their boundary integral equations, Numer. Math., 67:365-389, 1994

[29] C. Lubich and A. Schädle, Fast convolution for non-reflecting boundary conditions, SIAM J. Sci. Comput., 24:161-182, 2002.

[30] J.N. Lyness and G. Sande, Algorithm 413: ENTCAF and ENTCRE: Evaluation of Normalized Taylor Coefficients of an Analytic Function, Comm. ACM 14:669-675, 1971.

[31] B. Mayfield, Non-local boundary conditions for the Schrödinger equation, Ph.D. thesis, University of Rhode Island, Providence, RI, 1989.

[32] C.A. Moyer, Numerov Extension of Transparent Boundary Conditions for the Schrödinger Equation in One Dimension - A Java Implementation, to appear in: Amer. J. Phys., 2003. 
[33] K.B. Oldham and J. Spanier, The Fractional Calculus - Differentiation and Integration to Arbitrary Order, Academic Press, Boston, 1974.

[34] J.S. Papadakis, Impedance formulation of the bottom boundary condition for the parabolic equation model in underwater acoustics, NORDA Parabolic Equation Workshop, NORDA Tech. Note 143, 1982.

[35] A. Quarteroni and A. Valli, Numerical Approximation of Partial Differential Equations, Springer, Berlin, 1994.

[36] V.S. Ryaben'kii, Exact transfer of difference boundary conditions, Functional Anal. Appl., 24:251-253, 1990.

[37] V.S. Ryaben'kii, Exact transfer of boundary conditions, Comput. Mech. of Solids., 1:129-145, 1990. in Russian.

[38] A. Schädle, Numerische Behandlung transparenter Randbedingungen für die SchrödingerGleichung, Masters Thesis, Universität Tübingen, 1998.

[39] A. Schädle, Non-reflecting boundary conditions for the two dimensional Schrödinger equation, Wave Motion., 35:181-188, 2002.

[40] A. Schädle, Ein schneller Faltungsalgorithmus für nichtreflektierende Randbedingungen, Ph.D. Thesis, Universität Tübingen, 2002.

[41] F. Schmidt and P. Deuflhard, Discrete transparent boundary conditions for the numerical solution of Fresnel's equation, Comput. Math. Appl., 29:53-76, 1995.

[42] F. Schmidt and D. Yevick, Discrete transparent boundary conditions for Schrödinger-type equations, J. Comp. Phys., 134:96-107, 1997.

[43] F. Schmidt, Construction of discrete transparent boundary conditions for Schrödinger-type equations, Surv. Math. Ind., 9:87-100, 1999.

[44] I.L. Sofronov, Conditions for complete transparency on the sphere for the three-dimensional wave equation, Russian Acad. Sci. Dokl. Math., 46:397-401, 1993.

[45] I.L. Sofronov, Artificial Boundary Conditions of Absolute Transparency for Two- and Threedimensional External Time-Dependent Scattering Problems, Euro. J. Appl. Math., 9:561588, 1998.

[46] I.L. Sofronov, Non-reflecting inflow and outflow in wind tunnel for transonic time-accurate simulation, J. Math. Anal. Appl., 221:92-115, 1998.

[47] L. Ting and M.J. Miksis, Exact Boundary Conditions for Scattering Problems, J. Acoust. Soc. Am., 80:1825-1827, 1986.

[48] F.D. Tappert, The parabolic approximation method, in Wave Propagation and Underwater Acoustics, Lecture Notes in Physics 70, eds. J.B. Keller and J.S. Papadakis, Springer, New York, 224-287, 1977.

[49] A. Zisowsky, Discrete Transparent Boundary Conditions for Systems of Evolution Equations, Ph.D. Thesis, Technische Universität Berlin, 2003 